

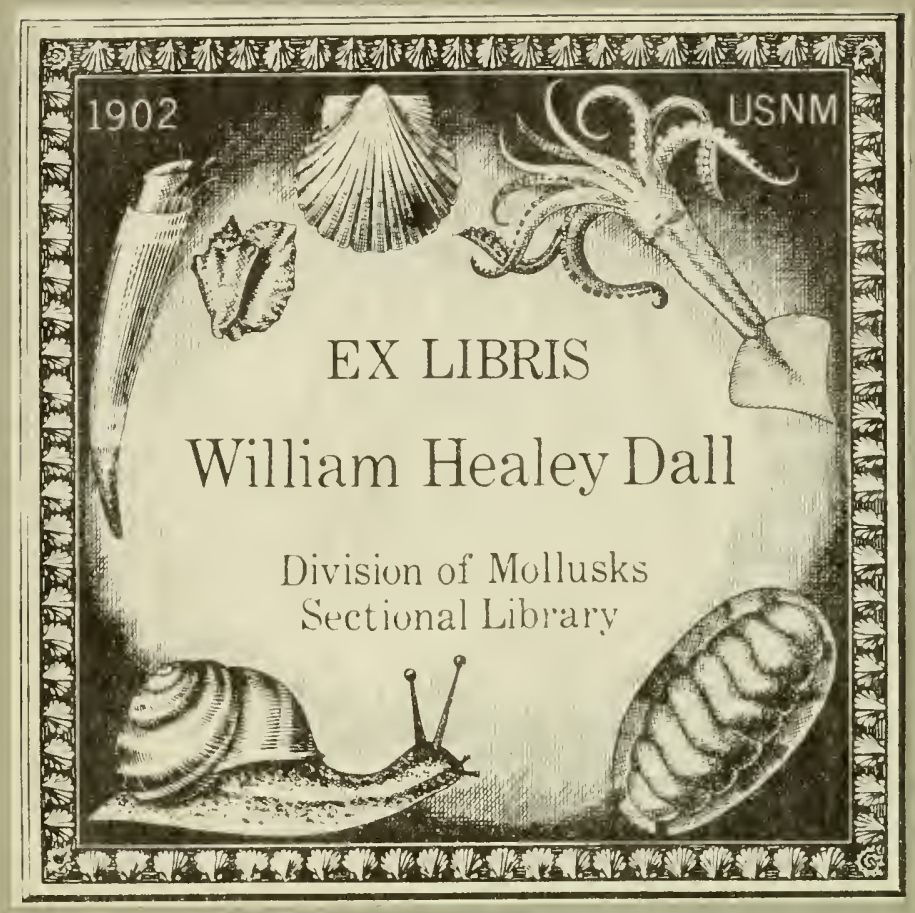


I BS.TON DViaton of Molluales 




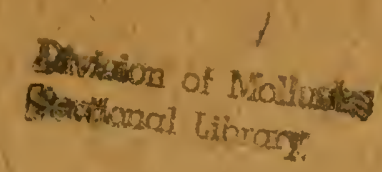

\title{
Mufeum Boltenianum
}

\author{
Give
}

Cacalogus cimeliorum

e tribus regnis naturx

qux

olim collegerae

Foa. Fried Bolten, M.D.p.d.

per XL. annos Proto phyficus Hamburgenfis.

Pars Secunda

continens

Conchylia five Teltacea univalvia, bivalvia \& multivalvia.

Ha mburgi,

Iypis Johan. Chrini. Trappii.

SMITHSCR.?.

MAY 21989

LIBRAE. D 

Reprint 1906. Seventy-five copies only printed, of which this is number $3 f$

$\lim _{2 \rightarrow \infty} 2+2$

$$
\text { ipdy hes }
$$

Ree'd May 26/06 


\section{INTRODUCTORY NOTE.}

J. F. Bolten's "Museum Boltenianum" is so rare a tract, that in response to numerous requests we have undertaken this reprint in photographic facsimile. Students of the Mollusca will now have ready access to the many changes of nomenclature therein contained.

The entire process of reproduction has been most carefully carried out by Mr. F. W. Reader, of Watford, whose labours we are pleased to have this opportunity of acknowledging. Owing to the poor quality of the original paper and the grey nature of the ink, which latter has in some cases failed to print in the original, numerous pages have been reproduced more than once before a sufficiently satisfactory result could be obtained.

The actual copy from which this reprint has been made formerly belonged to J. C. H. Crosse, of Paris; at his sale it passed into the hands of C. Davies Sherborn, and now forms part of the Geological Library of the British Museum (Natural History).

C. Davies Sherborn.

E. R. SyKes.

March, I 906. 



\title{
Mufeum Boltenianum
}

\section{$2 / 625$,}

Give

Cacalogus cimeliorum

e tribus regnis naturx

qux

olim collegerae

Foa. Fried Bolten, M.D.p.d. per XL. aanus Proco phyficus Hamburgenfis.

\section{Pars Secunda}

\author{
continens
}

Conchylia five Teltacea univalvia, bivalvia \& multivalvia.

Hamburgi,

Typis Johan. Chrifti. Trappii. 



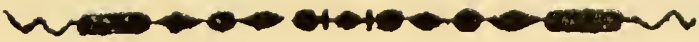

\section{B. S.}

Prater innumeros alios thefauros rerum naturalium, quas Vir Experientiffimus atque doctiflimus Boltenius Medic. Doct. \& Poliz. ter primarius, de republica litteraria pariter atque civili univerla, in primis vero de Hamburgenfí aternum promeritus, per quadraginta annos collegerat, immenfam reliquit conchyliorum, magna ex parte rariflimorum eorumque fplendidiflimorum \& optime confervacorum fupellectilem.

Cuius indicem fyftematicum ab ipfo beato poffeftore quam diligentifime curatiffime que elaboratum edituri heredes id mihi negotii dederunt, ut a Viro univerfa hifto. riz uaturalis prxfertim vero conchyliologix aınariffimo, peritifimoque Perro Friederico Rading/magna follertia revifum \& fynonymia locupletiore adauctum, brevi prafatio. 
ne Latina inftruerem, Quod quidem, ut. pore innumeris benevolentix monumentis ab ornatifiına famlia Bolreniana devinctus, licet minime pro conchyliologo me venditare aufim, atramen recufare nolui. Offendencur enin forlan primo obtutu conchyliologix arbitri pariter alque amantes rot novis iniauditisque momın bus, prafertim genericis in hoc caraligo obviis. Qua de re paucis monendi lunt, hac lingularis publicaque auctoritue carens nonenclatura unde origi, nens traxerit. Elaboraverat nempe Preclarilfimus Boltenius novuus \& proprium Syfte. ma narurale .conchpliologix, ab omnibus aliis priorum auctorum fyltemaibus malrum diverfum, idque adeo fubuliter adornaturn in artis certa five difeiplina formam. fecun. dum pecuiaris philofophix conchyliologicx regulas exadificatam conltructanque, redegerat, ut olim edita hac methodo fua naturali ftudendi hiftorix conchyliorum, propemodum inftar dictaroris vel alterius Linnxi in hac parte nature regnaturus videretur. Prodierunt re ipfa loco fpeoiminis atiquet plagula a Viro experienrifimo doctiffrmoque Dominico Schulize, M. D. acuto natarx ve. rique indagatore, fub ipfis beati Boltemis aulpicis adumbralx, Sed ipfum opus inte- 
grum proh dolor! acerha Schailzii morto interrupram, quum laboribas oscupara difractaque lenectus Bolterio impedimento efler, quo ininus folus limarer cumque orbe litrerato communicaret, follertem atque in. credibili fagscitate elucubraram methodum conchylıologicam. Curus quidem funda. mentuin lolidum nirebatur fuperfiructum. idex limplicl, eique egregix: naturam nemre offea reftaceorum regunienra varia condiruran inirium fecifle a fimplici \& plano regimine arque inde magis magisque sorra volumine ad rehignas fenfim elegantiores in. tricarioresque formas univalvium, bivalvium \& multivalviuin conchvliorum perrexiffe. ingeniofurn lane, auctureque rum natura iplius run! Syftematus condignum comnen. țum! Vunam bearas Boltensus reliquifer aliquan ordium \& generun uaturalitm, ann quirs reftacea ex hoc principio diftinguendi dilaberentur fciagraphiaiu. quam prafarioni noftrx inferere poruiffemus in magnum vfun eorum, qui catalogum fequenrem cum in. telligentia perlegere dignati forent Nunc vero proh dolur nil nifi nova teltaceorum genera, nifi omnia, pluruma certe ex profunda folidaque fsientia conchyliologica conPtituta, tamquam difiectı membra fyfteina- 


\section{VI}

rici apparent. In quo, erit lector, in de. licis modo habens reftas ob formarum \& colorum varietatem, hallucinari facile porerit, prafertum vibs a fynonyma defertus. errat circa fpecies determunandas, multum samen proficiet gnarus \& vere fyftesiaticus arbiter, quando Bolten" genera cum Linnai aliorumgue auctorum claffificationibus comparabir Nihil certe magis oprandum forer, nuli, ve integer hic teftaceorum thelaurus neurignam auctione diftractus, in manus talis viri venures, qui incredibiles labores ques Boltenius noner in comparando \& adornando hoc minenfo mufeo cenchyliologico exantlavit, in honorem fummi numinis, \& emolumentusn hiftorix naturalis convertere poflit, ac velit. Cetera, lećtors, conchyinorum amanti fcitu neceffaria, patebunt ex mlequentu prafusione vernacula. Dabam Hamburgi d. X. menl. Septcmbr. 1798.

M. Ant. Aug. Henr. Lichtenftein.

Vorrede. 


\section{Vorrede.}

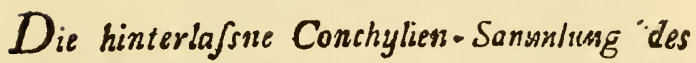
verforbenen Herrn Dotior ans Phyfacus J. F. Bolten, aft gewy/s in nuancker Hir. ficht fohr fichäzbar, und zesehnet iich durch äufferft Jeltene Stiuche ous. Wor: ser noch fehr vieiz sucht befthrubers frodo. haupt/ächlich abor enthält foltbe einen grof: fen Vorrath von meuentdeckten Sïdländern Der feelige Dr. Bolten fuchte fenze Sammalures fo vollftärsdig all. machen, als wöglich war, and or erlangte durch fene auswörtige grofje Bekatzntfchaft, durch Taufich wrid vuele Koften, eine Sammilung, die keines weute. ren Lobes bedarf. Der Kenner wird durch die kurze Benennung etnugermajen deren Werth beftumssen könness. Weat neehr abes' gevount folche bey der Anficht, weil haupsio fäthlich auf Farbe fowohl. als auch ouf Vollftändigktit der Stücke gehalten wordss.

Die grolfe Mannugfoltigknt bewog den Befitzer. feun engenes Syfem eu wähleno or benannte folche, wie das Verzeichniss zegget. mit lateing fchen und teutfchen Nahmen, fo gliscklich und gut diefe abir auch giswöhlt fard, fo müfsten doch manche den Ausländern ganz unbekannt blesben. Aus Liebe zur Natwrgefshuchte ïLernatm sets dieje 


\section{VIII}

dirfe Arbeit, und fügte die lateinifche Bonennung nach Gmelins 13ter Ausgabe des Linncifchen Syfferss. wre auch manshe Nachwoifung, wo folthe nur abgebildet war, bey: bey monchen mufste es leider unterbleibun, wo entweder gar keine Abzeichnung nachgezeigt werden konnte, oder wo auch folche fehr undeutlich antgegeben woorden. Die Einfchaaligten find an der Zahl beynahe 7000, und dis Zwey- und Vielfchaatigten an 1300. Bey einer fo grofent Menge -hoffe ich Verzetbung zu erhalten, wenn manche Stïcke nicht fo aufgefucht worden, wie es billig hätte feyn follen.

Es wiirde immer Schade Jeyn. venenn eine fo reiche ausgefuchte Sammlung das Schickfal fo mancher hätte, näm.uch verernztlt zu werden. Die Familie wïnfrht daher socht fehr enzen Köneer zu haben, mit ders wane fich gewrss billig finden würde, und crfuche deshalb fich baldigft zu melden. Noch word angezenget, dass die Conchylien durchgehends von gefthckter Hand vom Seefchmutz gereiniget find.

Im Monat September 1798.

\section{Pecer Friedrich Röding.}




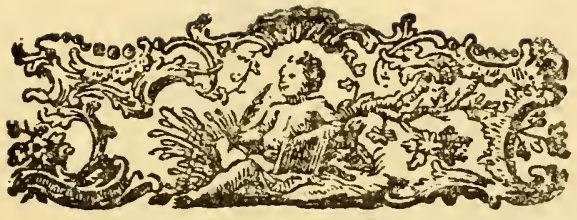

\section{Lade $\mathbf{I}$.}

\section{Patella. Schüfel - Mufchel.}

1. Perfuratio. Durchbohrte.

- Foramine centrali. Die Oefnung im Mitcelpunst.

1. Sulcatae. Gefurchie.

I 1 P. Mirella. Die Haube. Gmel. P. nimbofa; Sp. 197. Mast. I I heil. Gg. $91 \& 92$. I Stück.

2

32 P. Grifee. Die afchgraue Patelle. Gmel. P. Caffra; ip. 201. Mastroi I Theil. rab. I1. fig 95 ISe.

43 P. Infundibulim. Der Trichcer. Gmel. [P. 65. P. infunditulum; Schröt D. Litcerat. 3. P 72. n. $136.1 \mathrm{Se}$,

54 P. Umbrofa. Die dunkle Parella. da Costa, tab. 7. fig 4. ISt.

6

5 P. Ninbaje. Die Cchwarze Schein Pa. relle. Gmel rp. 196. P. nimbofa. Knort Vergn. d. Augen IVI tab. 29. fig. 4. Mas. tins I. tab. II. fig. 90 . 8 St

6 P. Radiata. Die Strabl.Patelle. Gmel. Sp. 144 . Parelle radiata. Chemn. Conch. 10. 9.329.1.168. fig.1618. ISt.

\section{Coftatce. Geribte.}

87 P. Limbasa. Dis gefäurare Parelle. Parella porgbyrozenias: P. 203. Martini ITh. rab. I2. fig. 102. 3. 4. $17 \mathrm{St}$. 


\section{$x+\frac{1}{2}+2$}

9 8 P. Rubeota. Die rörhliche Parelle. Parella refea: fp. 204. Martini I. rab. 12. fig. 1054 St.

10 9 P Vaviegat a. Die geripp:e Parelle. I St. il ro P. Veridena. Die grude Patelle. $3 \mathrm{Se}$. 12 II P. Viridss. Die grüolicbe Patelle. I St. 3. Clathrate. Gegitterte.

13 12.P. Rudis. Die plumpe Parelle. Gmel. Parella jamoicenfis; Ip. 200 . Martini 1. tab. I r. fig. 94. Lifter Conch. 1. 2. 528. ค. $6.3 \mathrm{St}$.

13 P. Cancellara Die gegicterte Patelle. Ginel. Pusella greca; Sp.J95. Marsini I. tab. 11. Iig 98.2 Si.

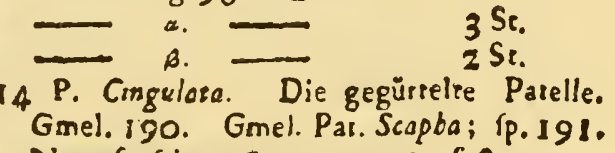

14 P. Cingulare. Die gegürrelee Parelle. Gmel. 190. Gmel. Par. Scapba; '́p.191. Naturforfsher 18. p. 10. t. 2. I.8.

4. Levignta. Glatre.

Is P. Ferula Die Schiese. da Coßta, t. 7. fig 7.2 St.

$1915 \% \mathrm{P}$ Purroletra. Die beftaubce Parella. $2 \mathrm{St}$.

\section{Patella. Schuifel. Mufchel.}

1. Perforatos. Durchbobrte.

- Foramime excentrico. Die Oefnang fectwărts,

1. Elevata. Erhöhte.

2016 P. Clauzs. Das Hŭnerauge. $2 \mathrm{St}$.

2117 P. Lacrymalis. Die Thranen-Parelle. $1 \mathrm{St}$.

22 is P. Graca Die griechilche Patelle. Gmel. Tp. I9s. Martins 1. tab. 11. fig 98.101. 104. Knors Vergn, 1. tab. 30. fig. $3.3 \mathrm{St}$. 


\section{4toratos}

2. Deprefir. Gedrikrel

23 19 P. Nummularis. Die Grofcheo oder weiffe Schlurfelloch Pateile. Gmel. Patella puftula; f f. 194. Chersn. 10. t.. 168. f. 163233.3 St.

20.P. Craviolaris. Die socbe SchlüfrllochParelle. Griel. Parella punula; fg. 194. ק. Martioi Befchr. Berl: Naturf. 2. i. i 2. f. 4. 5. Parella unguis. Pesiv Gaz. เab. 3. fig. 12. I St.

21 P. Puftula. Die Blatter. Gniel. rp.194. Chemn. Conch. 10. p. 338.t. 168 . fig. 1632. 33. Schröt. Einleisung in die Conch.2. 2. 5. Gig. 8. Lifter 528. fig. 3. I St.

\section{Patella. Schüffel-Murchel.}

2. Imperforata. Undurchbohrte.

- Margine edextujo, mil ungezdhnten Rand.

1. Vertice, roftrato, mit gefchnörkelten

Wirbel.

*. Uncinazc. Hakenförmige.

22 P Hungarice. Die Zoors. Kappe Gmel. 1p. 89. Patella ungarica. Liffer Conch. r. 544. r. 32. Knorr Verg 6. rab. I6. fig 3. Martiai 1. tab. 12 fig. 107. 108. 6 St.

27 2; P Pansouica. Die Narren Kappe. Lifter t. $545 .{ }^{f} 37$ 2St.

23 - P. Figura Die eingefchnicene Parelle. Gimel. 192 Lifter Conch. I 543 f 28. Martini I. tab. 12 fig. 109.110. ISt.

23 " P. Tricerinata. D e Grenadies-Müeze. Gmel. \{́p. 92. Schiörer Eıol. in die Conch. เab. 5. fig. 2 . da ofla tab.4. fig.9. $4_{\text {St. }}$ 


\section{Lethothers.}

30124 P. Cornu Copier. Das Fülltorn, da Cofa sob. 5. hig. 7 . 4 St.

3525 P. Rugofa. Die runzlicle Parelle, Gmel. Parella mitrula; 'p. 82 . Lifter : 544. f. 31. Maitini 1. 136. 12. fig. 111.112. 2 Si.

$26 \mathrm{P}$ Loctec, Die Nachikappe. Gmel. Sp.224. Patella lactea. Schröt n. Listrer. 3. P. 152. 0 294. Lister tab. 544. fig. 32. Pavande tab. 4. fig. B ISt.
B. Labiesce. Gezüngelee.

27 P, Egueftris. Die Fifchweiber-Haube. Fmel. ip. 1. P. equeftris Airgenv. Conch. *. 2. F. IR Cabochon, Marrini 1. sab 13. 6. 117.118. I St. 28 P. Undulara. Dle Matrofea-Mürze. P. equeftris. Gmel. Sp. Io (mit Aumpfes Spitze) Matsini I.t. I 3. fig 119.120 .35 t. 29 P. Creceulara. Die Giocke. Gualshi, rab. 9. 6ig. X. 2 St.

30 P. Conzorsa. Die Ziczack. Pacclle. Gmel. Parella contorta; fo. I8. Naturfoifch. 9. p. 199. c. 3. f 3. 4. Kämnerer Mufeum P. Radolte. p. 23. tab. 2.198 .2 Se.

31 P. Spiralis. Die Schneckea - Parelle. Gmel. Patella conzorta; $\gamma_{2}, 11$. da Cofta rab. 6. 6g.2. Ist.

32 P. Dispar. Die gezüngelte Kappe. Gmel. Patella contorra ; Tp. I1. Nasurforfeb. 18 St. tab. 2. fig. 13. 4 St.

33 P. Turcica Der Turban Gmel Parella finenlis; \{p. 3. Martini I. zab. 13. fig. 123. 124. 3 Se.

40 34 P. EleEtrita. Die Bernftein. Parelle. Gmel. Parella finentis; 'p. 3. 2 St.

4135 P. TEttum. Chinenfe Das cbioefifche Dach. Ginel. 'p. 1. Equeftis. Knorr Verg. 6. 8. 35. fig. 4. Martisi 1. tab.13. 


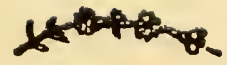

6g. 125. 126. Cbemn. 10. 13b. 168. $1630.31 .2 \mathrm{St}$.

36 P. Cbiwenfss. Der Regeo-Schirm Gmel. Patella finentis; 'p. 3 Argenv, tab. 2. Ge. F Matrini 1. ab 13. fig. 121.122. 4 Stücke.

43

37 P Mammula, Die Bruft. Warze. Gme!. Parella mammillaris; $\Gamma_{p} 91$. Lifter c. 537. f. 17 . Favanne tab. 6. fig. 63. 35:.

38 P. Plicasa. Der Kragen Det Kragen. Gmel, 'p. 13. Patella plicata. Nacutforfcluer 18.tab.2. fig. 12.12a. Favanne rab. 4 Gg. CC. 2. 2.1 St.

39 ! B. Hispida. Die Srachel Parelle. Gme!. Pacella aculeata; . p. 6. Favanoe tab. 4 . fig. B. 3. I Se.

r. Nerisoidec. Kammerförmige.

40 P. Fornicara. Die Pantoffel - Patelle. Gmel. Pacella Foroicaea; Tp. $^{5}$. Knorr 6. 1.21. fig. 3. Matrini 1. 28 b. 13. fig. 129. 130. $5 \mathrm{Si}$.

47 41 P. Porcellenc: Die Porzellan-Patelle. Ginel. Parella porcellana; fp. 4. Martini I. rab. I 3. fig 127. 128. Chemn. Couch. 9. sab. $124.1082 .5 \mathrm{Sc}$

42 P. Nuclens Die Kera-Parelle. Gmel, Pacella potcellana; f 4 . Favanoa tab. 4. fig E. 2. 3 St

43 P. Crepida. Die durcblicheige Parelle. Gmel, 'p. 17. Crepidula. Adanl. Serreg. cab. 2 fig. 9. Gasnot. Favanoe rab. 4 . fig. G.22, St.

so 44 !P Cymbeala. Das Kāhochea. I St.

5145 ! P. Appendierileta. Die Zunge. ISt.

5246 ! P. Rericulasas Die Netz-Parelle. da Colts. rab. 6 fig 4.2 St.

$5 3 \longdiv { 4 7 }$ P. Haliorordá Die gef́naörkelre Parellz. 
Gmel. Parella aculeata; rp. 6. Chemn. 10. tab. 168. fig. 1624. 25 . da Colta. sab. 6. fig. 1. J. $4 \mathrm{St}$.

No. 44.45 und 46 . find feht feltene Srüeke.

2. Vertiec abjoleto. Mit unkenntlichen

\section{Wirbel}

«. Ovaro foliacer Eyrund blattörmig.

47 * ! P Umbracula Der Sonnen-Schirm. Gmel. Pacella umbellata; fp. 146. Mar. tini 1.tab. 6 fig. 44 Chemn. 10.tab 169. fig. $1645 \cdot 46.3 \mathrm{St}$.

rehr felten.

\section{Lade 2.}

3. Ovato compreffe. Eyrund, feitwärts gedrückie.

48 P. Compreffa. Der Ellbogen. Gmel. Parella compreffa; $r_{p}$. 136 . Knotr Verg. 6.t. 28 f. I. Lifter t. 54 1. f. 25 Mar. tini 1. tab. 12. Ag. 106. Knots 6. 5.28. f. I. $5 \mathrm{Se}$.

49 P. Lucea. Der gelbe Hut, v. Born, tab. 18 fig 8 . 2 St.

50 P. Stvicta Der braune Hut. I St.

5 I ! P. Ulna. Die Wanne; viel ähnliches von $P$. umbella; $f$. 7 I. nur ift diefes Exemplar mehr gewölbe wie die Ellbogen- $\mathrm{P}_{\mathbf{a}}$. ielle. I Si.

rehr felten.

52 ! P. Gondola. Die Gondel. da Cofta tab. 3. fig.9 ISe. fehr felten.

60

53 P. Pectinata. Die Kamm- Patelle. Gmel. fp. 93. Schrörer Einl, in d. Conch. tab 5. 6. 3. Martini I, tab. II. fig. II5. I I6. S. St, 


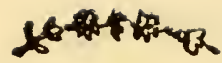

y. Ovaso fimplices. Eyrund einfacbe.

54 P. Smaragdina. Der Smaragd. da Costa tab. 4. fig. 4 4. 5 St.

55 P. Micans. Die Schimmer-Patelle. Schroter Einleitung in Conch. 2. tab. 5. fig. 13. 9 St.

56 P Pellucida Die durchfichrige Patelle. Gmel. 133. Knorr Verg. 6. t. 28. f. 6. Chemn. Conch. 10. t. 168. Ag. 1620.21. v. Born tab, 18. fig. 92 St.

64

57 P. Teftudinaria. Die Schildktöten-Patelle. Gmel. 134. Knors Verg. 1. tab. 2 i. f. 1 \& 3. t. 30. f. 2-5. Argenv. Conch. 8. 2. f. P. Martini 1. เab. 6 . fig. $45-48$. 9 St.

65

58 P Intersexzc. Das Gewebe. Gmel. 135. tefudiualis; Chemn. Conch. 10. t. 168. f. 1614 is. Favanne lab. J. fig Q 2. 8 St.

59 P. Diopbang. Die Warze. Lifter 8. 543. f. 26. A. 2 St.

60 P. Nova Zeelandia. Die Neu SeelandsPatelle. 7 St.

68

61 P. Tairenfis. Die Otbaheite. Favanne sab. 1. fig. N. N. 3 St.

69

62 P. Capillacea. Die Haze Parelle. Gmel. 176. Martini 1. tab 7. fig. 58. 59. 4 St.

70 $6{ }^{4}$ P. Digirale. Der Fingerhut. Gmel. \{p. 176. 6. Martini J.tab.7. f.57. 6St.

7164 P. Capillata. Gmel. Ip. 176. Martini fig.56.57 It.

7265 P. Papillaris. Die dicklinigte Parelle. Gmel. P. leucopleura; Ip. 34 Lifter t. 539. f. 22 Knorr 6. t. 28 . f. 9. 1 Si. 73 66 P. Morio. Det Mohs, ISt, 
$74[67$ P. Africena. Die anicanifche Parelle. $2 \mathrm{St}$.

7568 P. Nigrite. Det Neger. Favadae sab.1. fig. B. I. I St.

76169 P. Bifurca. Die Gabel. 2 Sr.

7770 P. Fnlgur. Die Blizz-Patelle. IS.

7871 F. Fungoides. DEI Filz. Lifter 539. fig. 232 St.

8072 P. Aranea. Die Spinae. 2 St.

8173 ४. Clypeus. Die Schild-Patelle 3 St.

8274 P. Crocer. Die rafrangelbe Patelle. P. croces; Gmel. Ip. I74. Schböter D. Lietaras. 3. P. 83. 0.159. J St.

83 75. P.Contus. Der Kegel. Gmel.P vulgara; Ip 23. Martini r. sab. 5. f. 38. Koort Verg 6.

8476 P. Cuprer. Die broncirse Patelle. Pats cuprea Gmel. Sp. 6I. I St.

8577 P. Cornea. Die Hurn.Parelle. 3 St.

8678 P. Oculus Cart. Das Katren-Auge. I St. febe feltea.

8779 P. Patere. Die Schale. Gmel. Pacella refludioarsa; Sp. 134. Mattini I. tab. 6. f. $45: \quad 1$ St.

79 * P. Aurora. Die Morgentöthe. I Se. fehr febön und felsen.

89

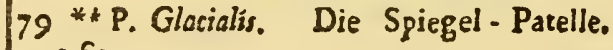
15 .

\section{Lade 3.}

90

80 P. Circkinfcripra. Die eingefafsre Parelle. Gmel. P. lurinamenfis; fp. 128. Matt. I เab. 7. fig. 50. $2 \mathrm{St}$.

81 P. Hidatis. Die Blafe. Lifter Conch. tab. 502. fig. 26. I St. 


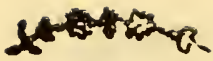

\section{Pateila. Schüfrel - Mulchel.}

\section{Imperforalce. Undurctbohrte.}

- Mergaze madulete. Mit gewelitem Rand.

\section{Simplices. Einfache.}

9282 P. Rogea. Die rofentothe Pazelle. Gmel Patella umbella; fo. 71 . Martini $1 .: 8$. Gg. $63.15 \mathrm{Se}$

83 P. Rubicunda. Die rothftrahlichte Pa. tella. Goel. Patella umivella; $\int_{p .} 71$. Marotini I. tab. 7 fig. 52.53. 32 St.

83 * P. Discer: Der Teller. Gmel, Parella unibella; 'p. 71. var. ISt.

84 P Variabilis. Die verāadnrliche Patelle, wie vorbergehende nar fant rofeorbch: mis braunen Siteifen. Gracl. P. umbella; ip. 71. var. 9 St.

85 P. Grifea: Die graue Patelle. Gmel. P. gifiea; Jp. 188. Adanf, Seneg. 1. t. 2. f. 5o 3 St.

86 P. Plumbea. Die Aahlgraue Patelle. Gmel P. margarinacea ; fp 80. Marcini.1. tab. 10. fig:8j. a 10 St.

87 P. Silicine. Die Feuerftein farbene $\mathrm{Pa}$ telle. Gmel. P. margaricacea ; fp. 80 . M:5tiai I. t. 10. 6g.85.b. 2 St.

\section{Lade 4.}

9988 P. Gigas. Die groffe Patelle. Favanne tab. 2 fig. D. 1.

89 P. Marmorea. Die marmorweiffe Pacelle. Favanne tab. 2. fig. D. 2. $3 \mathrm{St}$.

10190 P Senilis. Die abgenutzte Patelle. Gmel Parella judica; f́. 127. Martial I 7 . fig. 49, I St. $4 \frac{1}{2}$ Zoll gtofs. 
$102 / 90 *$ P. Crepidula.

103 - Martini I. tab 8.

104
105
106
107 $9_{92} \begin{aligned} & \text { P. Nodofa } \\ & 92 . \text { Punctulata }\end{aligned}$

Gmel. (p. 68 \& 132. Patella punftulata; 'p. 68 Martini Conch. 1.t.7. fig 55. Lifter 537 . fig 20.3 St.

108 . 93 P. Ajpera Die rauhe Patelle. Faranoe tab. 2 . fig G. $2 . \quad 3$ St.

$109 / 93$ - P. Solaris. Die Sonnenftrahlen Parelle. Gmel.P. tenuis; fp. 8I. Martiai 1. t. 10. fig. 87. I St.

11093 P. Luratis Die Mond Pacelle. I St. II 94 P. Nubila. Die wolkigre Parelle. 3 St. 11295 P. Cerasa. Die wachsgelbe Patelle. 2 St. 11396 P. Ruffica. Diegemeine Patelle. Gmel. P. ruftica; fp. 137. Schröter Einleit. in Cooch. 2. p. 428. t. 5. F. 4. Schrôter n. Litter. 3. P. 108. 208-2 I I. Gualti. tab. 8 Sig. P. Gualti tab. 9. Sig. C.

$$
\text { - } 8 \text { St. }
$$
$115 \overline{30.68}_{5} \beta_{16 \mathrm{St}}$ Mnort Verg. 3. $\mathrm{rab}$.

11697 P. Squamata. Die fchuppigte Patelle. Gmel. P. Iufitanica; (p. 123. A) Martio ni fig 35. a. b. 2 St.

11798 P. Granuleris. Die kornichee Pacelle.

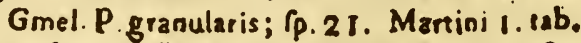
8. fig.61. Lifter Conch. 536.f. 15. 9 St.

1899 P. Fulva. Die rothgelbe Patelle. Gmel. fulva; Pp. 102. 2 St.

119100 P. Magellanica. Die Magellanifche Pa. telle. Gmel.P. magelanica; Sp. 52. Gualt. teft. t. 9. f. 8. Martini 8. tab. 5. fig. 40. a. b. 

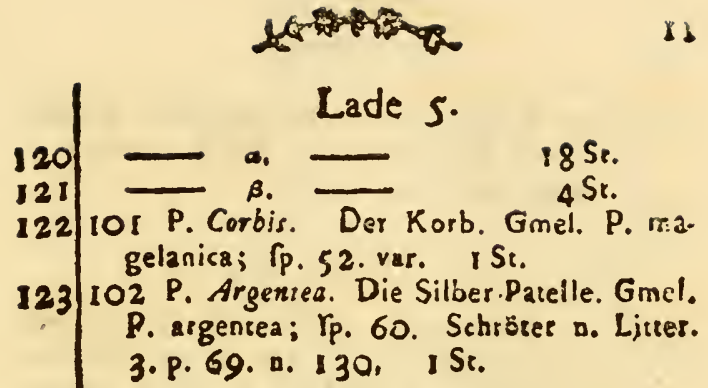

2. Appendiculata. Angeferze.

124103 P. Cocblear. Det Pferdekopf. Gmel. fp. IS 5. Schröter n. Litter. 3.P. 105. n. 204. Korr 2 tab. 26. Gg. 3. 5 St.

12.5104 P. Rudicula. Der Löffel. Favaone tab 97. fig. B. I St.

\section{Patella. Schüfrel-Mufchel.}

2. Imperforatce. Undurchbohrte.

-es Mergize deudaro. Mit gezahneltem Read.

1. Polygone. Vielwioklichte.

126 Ios P Medufe. Das Medulen Haupr. Gmel. P. feruginea; Ip 73. Knorr Verg. 3 tsb. 30 fg. I Martini I t.8.fig.66. I2 St. 127106 P. Fysea Die dunkelbraune Patelle. Gmel. P fufea; fp. 138 . I St.

128107 P. Modulus. Die Form-Patelle. Gmel. P. monopis; fo 78. fehr felten. A) Li. fier Conch. $2 a b .532$. fig. I 1 . I St.

129108 P. Laciniofa. Die vielftrahligre Parelle. Gmel. If 18. Knorr Verg. 6. t. 30. fig. 2-4. Martioi Conch. 1. 10 . fig 81. $2 \mathrm{St}$

130109 P. Lamellata. Die blätrichte Patelle. ros Buí iab. 28, fg. I. 6 St. 
I3I/10 P. Spinerum Die dornichte Patelle. Gmel. P. barbara; fp. 20. Knotr Verg. 5. tab. 13 . fig. 5 . 4 St.

\section{Lade 6.}

I32 II I P. Granatina. Das Bocks-Auge. Gmel.

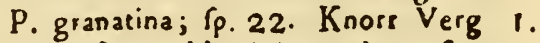
t. 30. fig. 2. Martini I. lab. 9. fig. 71 . 72. $21 . \mathrm{St}$.

$133112 \mathrm{P}$ Munitialis. Die Feftungs. Patelle. Favanne tab. 2. fig. B.2. $3 \mathrm{St}$.

134 II3 P. Caftrenfis. Die Lager. Patelle. 9 Se.

135114 P. Obfidionalis. Die Hornwerks. P2. telle. II St.

I36 I 15 P. Canaliculata. Die ausgeböhlie Parelle $3 \mathrm{St}$

I 37 I16 P. Depreffa. Die fache Patelle. Knorr 4. rab. 30. fig. 4. 5 St.

138117 P. Nivalis. Die Milch-Patelle. 4 St. 139118 P. Sidus. Die Geftira. Parelle. -

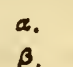

1 Se.
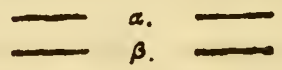

2 St.

2. Stellata. Sternförmige.

J42 119 P. Corrufca. Die Glanz. Parelle. ISr.

143120 P. Radians. Die Sirahl. Patelle. Knorp Vergn 3. tab. 29. fig 3. $6 \mathrm{St}$.

14412 I P. Afrolepas Die Siern. Parelle. Gmel. P. monopis. fp. 78. Martini I. 1. 9. fig 80. 4 St.

145122 P. Polaris. Der Polarftern. Gualtiv. rab 9. Ag. F. S St.

146123 P. Stellaris. Der Ordenftern. Gmel. raccharina; fp. 19 Lifter Conch. tab. 532 . Gg. 10. Favanne cab.2. Gg 2.3. $4 \mathrm{St}$. 


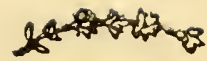

\section{Lade 7.}

Haliotis. See-Ohr.

I47 I H. Gigas. Das groffe See-Ohr. Gmel. \{p. 18. gigantea.Cheinn Conch. 10.1 167. fig. 1610.11. I Siück. grofs 6 Zoll.

$1482 \mathrm{H}$. Elephanezra Das Elephanten Ohr.

$1493 \mathrm{H}$. Iris. Das Regenbogen See. Ohr. Gmel fp. 19. Chemn, 10. 1ab. 167. fig. 1613.5 St.

$1504 \mathrm{H}$. Mide. Das Midas. Ohr. Gmel. Sp. I. Mattini I. tab. 14. fig. 136. Gualt. tab. 69. fig. B B. 4 St.

ISI $5 \mathrm{H}$. Tuberculate Das knolligte See Ohr. Cmel. 'p. 2. Martini I tab. I5. 16. 6g. 145-149. $18 \mathrm{St}$.

\section{Lade 8.}

$1526 \mathrm{H}$. Sirtata. Das geftreifre See Ohr. Gmel. ip. 3. Martini Conch. 1. t. 14. f. 138. Knorr Verga. t. 17 fig. 2.3. $21 \mathrm{St}$.

$1537 \mathrm{H}$ Cocblear. Das löffelförmige See-Ohe. I St.

1548 H. Rugofa. Das sunzlichte See-Ohr. 3 St. Ij59 H. Plicara. Das gefaltene See.Ohr. Gmel. [p. I3. Schräter Einleit. in VerAtei. 4. p. 278. t. 3. f. 9.2 St

$15610 \mathrm{H}$. Rudis. Das plumpe See-Ohr. I Se.

I57 II H. Calata. Das gemeiffelse See. Ohr. Gmel. fp. 17. ovina Chemn. 10. t. 166. fig. $1609.3 \mathrm{St}$.

$15812 \mathrm{H}$. Incrustata. Das gedeckte See-Ohr. 2 St.

I59 $13 \mathrm{H}$. Cingulata. Das gegürtelte See-Ohe. Gmel. Ip. 7. parva das grüne. Mart. 1. tab. 14. fig. 1 40. Knorr Verg. 1. tab. 20. fig. 5. 2 St. 
160/14 H Rubicunde. Das rothe See. Ohr. Gmel. Sp. 7. patva Knost Verg. I. tab. 20. fig. 5. Martini 1. tab. 14. fig. 1/a 3 St.

$15^{3} \mathrm{H}$ Aleata. Das würffichre See - Ohr. Gmel. Sp 9. $\mathrm{H}$ auftralis Chemn. 10. tab. 166 fig. 1603.1604 . I Sh

$16 \mathrm{H}$ Virginea. Das niedliche See. Ohr. Gmel sp. 16. Chemn, 10. tab. 166. fig. 1607 1608. 2 St.

163 I7 H. Granulata. Das bucklichte See Ohr. Gmel. H. varia Ip. 4 Martini 3. tab 15. fig 144.6 St.

164 18 H. Marmorala. Da; gemarmelte SeeOhr. Gmel. Ip 5.H marmorata Knorr Verg. 2 t. 17, f. 4 5. Martini 1.tab. 14. 6g 139. 145. 10 St.

$16519 \mathrm{H}$. Pilta. Das unvergleichliche See Ohr. Gmel. fp. 14. H.glabra Chemo. JO. tab. 166 fig 1602 . I St.

I66 $20 \mathrm{H}$. Afining Das Elets Obr. Gmel.rp.6. Martini I. tab. 16. fig 150 . Knors Verg. 3.t. I6. fig. I.

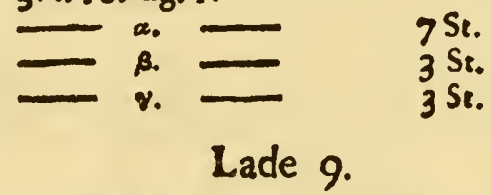

Sinum. Milch-Näpchen.

I70. I S. Fuscum. Das weilsbraune Milch - Nappchen. Gmel. Helix. 'p. 152 haliotoidea Knorr Vergn. 4. 5 17. fig. 5. Martini 1. tab. 16. fig. $151-154$. $5 \mathrm{St}$.

IyJ 2 S. Haliotoideum. Das See-Ohr rörmige Milch-Näpchen. Gmel. Helix haliotoidea fp. I52. Knort Vergn. 6. 1. 39 fig. 5. $5 \mathrm{St}$.

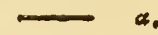

ISt. 
Bulla. Die Blafe.

173 I B. Aperra Die durchíchtige Blafe Gmet. rp. 8. Martini Conch. 1. P 266 vign. 83. fig. 3. Chemn Conch 10.t. 146. fir. 1354. 1355. Gualti. tab. 13. fig. $\mathrm{E} \mathrm{E}$ 4 St.

1742 B. Naucum Die Schwimm Blale Gmel. fp. 7. Knorr Verg 6. t. 38. fig. 2. 3. Martini I. tab. 22 fig 200201.4 S:

1753 B. Hydatis. Die Waller - Blafe. Gracl. rp. 9. Chemn. 9.t.118. f.rolg. Martini I tab. 21 . fig 199. $6 \mathrm{St}$.

$176 / 4$ B. Cylindrica. Die cylinderförmige Blafe. Gmel. ip .38. Chemn. Io 1. 146 . . 1356. 57. Linter 714. fig. 70.2 St.

1775 B. Lignaria Der Zrollkuchen. Gmel. fp. II. Knort Verg. 6.: 37. \{. 4.5 . Martini t 2T. fig 194 I95. 7 St.

1786 B Umbilicata. Das lange Kibitz-Ey. Gmel. Ip. IO. ampulla 3. Martini J. tab. 22. fig. $202-204.3 \mathrm{Si}$

$179 / 7$ B. Pbyfis. Die-zerbrechliche Blafe. Gmel. [p. 12. Argenv. Conch. t. 17 fig. 1. Martini I. tab.21. fig $196.197 .6 \mathrm{St}$.

1808 B. Cingulara. Die gegurrelte Blare. Gmel. Sp. 36 Velum. Chemo. 10. 1. 146. fig. 1348 49. Martin I. cab. 2 1. fig. 198. $4 \mathrm{St}$.

1819 B. Ouum Vanelli Das Kibitz.Ey. Gmel. [p. I0. Ampulla. Martini 1. t. 2 I. fig. $188.189 \quad 12 \mathrm{St}$.

38210 B. Ampulla Sirrara. Das gefteifte Ki. birz Ey Gmel. Sp. 10. Ampulla $S \mathrm{Se}$

183 I B Fascrata. Das gebānde't Kibitz Ey. Gmel. Ip 10 Ampulla. Martini 1. 2 . 1. fig 190. 19I. 5 St.

184 r 2 B. Buila Rubra Das rothe Kibitz. Ey. Gmel. (p, Jo. Ampulla $14 \mathrm{St}$ 
$185 \mid 13$ B. Flommea Das geflammre Kibitz-Ey. Gmel.'P 10 Ampulla. 2 St.

Nerita. Halbe Mond -Schnecke.

- Cleustro glabro. Mit glatter Decke,

I Labio crenutoso Clovicula elongare.

Mit gezahneltes Lippe und verlängerter

Spindel.

1861 N. Corona. Die Dornen-Krone, Gmel. N. fp. 26 Rumpf mul, L. 22, f. O. Maltinig. t. 124. 6g. 1083. 1984. 2 St.

187 N. Spinula. Die Rachlichte Nerite, Gmel. N. corona; fp. 26. var. 2.Se.

1882 N. Serpentine. Die Schlangen. Nerite. Geve tab. 24 fig. 250-52. Lifter 606. fig. 35. 11 St.

1893 N. Cardinalis. Die Purpur. Nerice. Gmel. Tp. 29. Auviatilis. Martini fig. 1088. h.i. I St.

1904 N. Latilinea. Die breirlinigte Nerire. Gmel. 'p. 29 Anviatilis. Martini 9. tab. 124. fig 1088. a. c.

1915 N. Poralella. Die gleichlinichte Nerite. Gmel, 'p. 29. Auviatilis $\delta$. Martini 9. tab 124. f. 1080.81 .

$1925^{*} \mathrm{~N}$ Ziczac. Die Zierack-Nerire. ISt.

1936 N. Usnea Die molsfarbige Nerite. Lifer 605. fig 33. ISt.

$1947 \mathrm{~N}$ Aracbnoidea. Die Spinoeweben. Ne. rite 1 St.

1958 N. Dubie. Die Bänder Nerire. Gmel. Ip. 34. Marriai 5. cab. 193. fig 2019. 2020. $8 \mathrm{St}$.

1969 N. Patella. Die Mürze. Gmel. N. viola. cea; fp. 68. Martini 1. t. 13. fig. I 33 . 134. 4 St. 


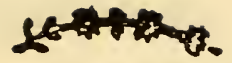

197/10 N. Hians. Die weitmündige Nerite. Lifter 604. fig 24. I St.

198 II N. Oculus. Die Augen. Nerite. Gmel. P. 35. pulligera. Martini 9. tab. 124. fig. 1078. 79. 2 St.

19912 N. Crocea. Die Saffran-Nerite. Geve tab 23. fig. 242. a \& b.

\section{Lade IO.}

2. Labio densato, clavicula deprefJa.

Mit gezabnelter Lippe und gedrückter Spiodel.

20013 N. Fusco fasciata. Die tothe Band - Nerice. 2 St.

20114 N. Allo Lineate. Die weife Band-Ne. site ISt

202 I5 N. Nigro cinkfa. Die fthwarz gebandete Nerite. Martini 9. tab 124. fig. 1089. List. $X .2$ St.

20316 N. Olivacea. Die gelb gebandere Nerite. 2 Se.

20417 N. Polisa. Die bunte Nesite. Gmel. fp. 43. Geve tab. 22. fig. 224. a \& b. Knorr Vergn. 3ter Theil. t. 1. fig. 4.

20518 N. Fulmmer. Die getammie Nerite. 1 St.

20618 N. Nebulofa. Die wolkigte Nerite. $3 \mathrm{St}$.

$20718^{* \hbar}$ N. Fascia lare. Die breire Binde. I St.

20819 N. Dendrites, Die gemahlte Nerite. I St.

20920 N. Monile. Die Ketten.Binde. I St.

21021 N. Aurautiace. Die Orange - Netite 
$21 / 22$ N. Opaca. Die helldunkle Nerise. Gmel.

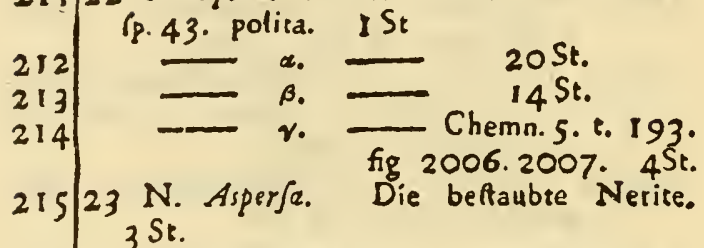
$21624^{3}$ N. Cbryfoforna. Die rothmündige Ne. rite $6 \mathrm{St}$.

21725 N. Arriaca. Der fehwarze Flor. Gmel. ip. 66. bifosciata. Chemn, 5. tab, 193. 2015. ISt.

Nerita. Halbe Mond Schnecke.

* Claufira murisato. Mit geperlter Decke.

Labio erexclaro rugofo clavicula deprefja.

Mit gezahoelter und gerunzeleer Lippe und gedruekter Spindel.

21826 N. Alabaftrina. Die weille Nerice. I St.

21927 N. Trariegata. Die rehäckigte Nerite. Gmel. Ip. 48 Groffa. Geve tab 23. fig. 239 a \& b. Martini 5. t. 191. 1968. 1969 . $5 \mathrm{St}$.

$22028 \mathrm{~N}$. Nutecula. Die rufzfarbene Nerite. $25 t$.

22129 M. Angulata. Die winkligte Nerite. Gmel. 'p.65 reflellata. Chemn. 5. t. 192. 1998. 19992 St.

$22230 \mathrm{~N}$. Imperferta. Die unvollendere Ne. rite Gmel. fp. 45 Albicilla. Chemn. 5 . t. 1932000 . a. b. c. d. Is St. 


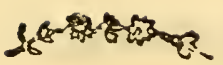

Nerita. Halbe Mond-Schnecke.

* Clauftro rkgofo murisato. Mit gerunzelecs

und geperlice Decke.

Labio rugofo-dentato clavicule elongara.

Mir gesunzelcer und gezähnelter Lippe, verlängerter Spiodel.

22331 N. Peloronta Die aufgeblafene Nerite. Gmel. Sp 44. B. 2 St.

22432 N Papilio. Der Schmetterling. Gmel Ip 44 Peloronca Chemn 5 tab 92.1977. $2 \mathrm{St}$.

$22533 \mathrm{~N}$ Cruenta. Die blutige Nerite $2 \mathrm{St}$

22634 N. Virginea Die Blumen - Nesite Ginel. Ip 44 Peloronta Chemn.5.t. 92 1978 - $1980.20 \mathrm{St}$.

$22735 \mathrm{~N}$ Mufica. Die Noten-Nerice. Gmel fp 63. flammea Chemn. 5. 1.192.1994 $7 \mathrm{St}$.

22836 N. Teffellata. Die würflige Nerice

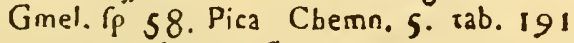
196419653 St.

$22937 \mathrm{~N}$ Mille punţata Die raufendfacl punctirre Nerite 1 St.

23038 N. Fascia DieBinde. I St.

23139 N. Maculnta Die gefleckre Nerite. 2 St.

$23240 \mathrm{~N}$ Pulcbra. Die fchône Nerite. I St.

23341 N. Nigrita. Die Neger-Nerite. Gimel. fp 46. Hiftrio Martini 5. t. I9I fig. 1960. 1061 II St.

$23542 \mathrm{~N}$ Confpurcara. Die befchmutze $\mathrm{Ne}_{\mathrm{e}}$ rite. I St.

23643 N. Chamereon Die vielfarbigte Nerite. Gmel. 'P. 49 Chemn. 5. tab 192. fig. 1988 I 99 1. Regenf, t. 3. fig. 26. I St. B 2 
237/44 N. Murscate. Die Arebligte Nerite. $2 \mathrm{Se}$

23845 N. Pecten. Die Kamm-Nerite. ISt. 23946 ! I N. Scbmideliana finiftrorsa, folfilis. Die linksgewundene Schmideliche Nerite. Gmel. Ip. 72 Chemn. Conch. 9. t. II 4. $2 \mathrm{St}$.

Ag. 975.76 . Naturforfcber 6.p. 165 .

\section{Lade II.}

24047 N. Nigreola. Die Ebenhoiz-Nerite. Gmel. 1p. 62. Antillatum. Chemn. S. tab 192.1987 I St.

24148 N. Eburnea. Die Elfenbein - Nerite. Gmel. 'p. 47. plicata. Favane tab. 10. fig. 0. 3. Lifter 595 . fig. 3. 5 St.

Albula. Halb-Nerite.

- Tefticulata. Hodenartige.

243 I A. Albumen. Die eyweiffe Halb. Nerite. Gmel. Nerita. 'p. S. Martini 5. t. 189. 1924. 1925. Knors Vergo 4. t. 7. f. 4:5. 3 St.

2442 A. Vitellus. Der Eydoster. Gmel. N. orientalis. Sp. 12. Chemn. 5. t. 188.1898. 1899. 8 St.

2453 A. Didyma. Der braune Hoden. Gmel, N. glaucina $\{p .3$. Favanne tab. 10. Lit. K. L. Chemn, 5. t. 186. fig. 1856.57. $6 \mathrm{St}$.

2464 A. Mammilla. Die Zitzen Halb-Nerıte. Gmel. N. S. 6. Cbemn. 5.t.189.1928 193 I. 9 St.

2475 A Adufia. Die angebrante Halb - Nor rite. 2 St. 


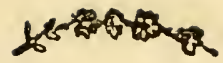

$248 \mid 6$ A. Aarantium. Die Pommeraze. Gmel.

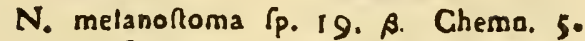
t. 189. fig. 1934. 1935. Koorr Vergo. 4. t.6. fig. 3. $4 .-2 \mathrm{St}$.

Albulc. Halb-Nerite.

* Umbilicata Genabelte.

7 A. Heparica. Die blasgelbe Halb-Nerire. Gmel. N melanoftoma fp. 19. Chemp. 5. rab. 189 fig 1932.33. I St.

2508 A Mammata. Die Bruft Drijfe. Gmel. N. melanoftoma í. 19. $x$ Cbema. 5. t. 189 fig. 1936.37 .7 St.

A. Fasciat a. Die gebandete $\mathrm{H}_{\mathbf{a}} \mathrm{lb}$ - Netite. Gmel. N. melanoftoma f́p. 19. .. Chemn. 5.1. 190. fig 1940.4J. I St.

$25210 \mathrm{~A}$. Trenquebarica. Die gläferne Halb. Nerite. Gmel. N. Papilla Yp. 20. Chemn. 5 tab. 189.1939. 2 St.

Cyphoma. Halb-Porcellane.

253 I C. Gibbofa. Der Höcker. Gmel. Bulla. f. 6. Martini 1. tab. 22. fig 213.214. Knorr 1. tab. 14. fig. 4. 13 St.

2542 C. Dorfata. Der Hochrücken. Gmel. Bulla gibbofa fp. 6. Martini 1. 1.22. fig. 211. 12. Knors 1. tab. 14. 6g. 3.

\section{Volva. Das Ey.}

2551 V. Cygnea. Das Schwanen - Ey , mik brauner Mündung. Gmel. Bulla Orum. Sp. 1. Martini 1. t. 22. f. 205. 206. 6 St.

2562 V. Ovum. Das Gânfe-Ey, dünnfchalige, mit weiffer Mündung. Gmel. Bulla ovum; SP. I. 5 St. 
$257 / 3$ V. Hyperemium. Das Wind Ey. I St. 25814 V. Biroftris. Der faltche Webertuhl. Gmel. Bulla. Kp. 3. Markini I. tab. 23. .217 a. b. Lifter 711 . fig 66 ISt.

2595 ! V. Textoria Der ächee Weberlpubl. Gmel. Bulla Volva. 'p. 2. Martini r. tab. 23 f. 2 I8. Knotr Verg. 5. t. I. f.2. 3. 6. t. 32. f. 1 .

2606 V.Perla. Die Perle. Gmel. B. verrucofa. f. 5. Martini 1. t. 23. f 220. 21. Lifter 7I2. fig.67. Kaors Vergn.4. 1. 26. f. 7 .

\section{Cypraa. Die Porcellane.}

* Globofe. Kugelformige.

I. Irregulares. Unausgewachfene.

261 I C. Ouum. Das Hüner-Ey Gmel Bulla ferruginofafp 35. Mart.1.1 22.209. $\beta$. ISt.

2622 C. Nivea. Das Tauben-Ey 2 St.

2633 C. Limbate. Die gefäume Porcellane. ISi. 2644 C. Fuliginofa Das geflammte Ey Gmel. B. ferruginofa ip 35. Martini I 1.22. f. 209. $210.2 \mathrm{St}$

265 s C. Pafcbalis. Das Oreer-Ey Gmel. Bulla ovata fp. 34 Martini 1. t. 22. $\{.207$ 208. Knorr Verga 6. t.18.5 2. t. 27.6.6. 2 St.

$266 / 5$ * C. Prunus. Die pflaumenförmige Potcellane. Gmel C. Pulla. fp. 61 Martini 1. täb. 26. f. 269270,2 St.

267 5 ** C Bullata Die gebandete Porcellane. Gmel. C cinerea Ip.16, Mare I tab.25. 5254 55. $1 \mathrm{St}$.

2685 C. Cartbaginenfis. Das Klip - Hoen Gmel. C. Mus. Ip. 43. Martini I. t. 23 - f. 222. 223. Knorr Vergn, 3. 8.12. .. 3. 3 St.

26977 G. Tuberofa Die puckligie Poncelline. ${ }_{3}$ St. 


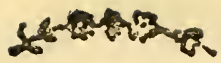

$27018 \mathrm{C}$. Maurisiane. Der grode Schlangen. Kopf. Gmel. Cypr. Pp. 41. Knorr 2. 1.37. fg. 5. Martini 1. 2. 30. f.317.319.

\section{Lade 12.}

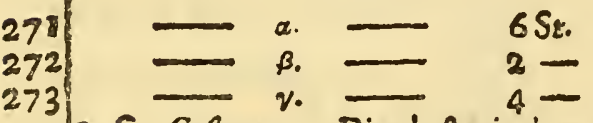

2749 C. Caftanea. Die kaftanienbraune Pozo cellane. Gmel C. Ip.41. var.

27510 C Caput Serpentis. Der kleine Schlan. genkop:. Gmel. C. Ip. 39 Knorr 4. rab. 9. fig. 3. Martini Conct. I. \&. 30. f. 316. 23 St

27611 C. Oculata. Die vielängigre Porcellane. Gmel. C. Histio rp. 120. Chemn. 10. t. 145. E. 1346.47. Favanne 3. t. 12. fig E.2. 2 St.

27712 C. Hiftrio. Der Harlekin Gmel. Ip. 120. Knors Vergn. tab. 16. fg. [. 2 St.

27813 C. Stellate. Die geftippelre Porcellanse. Gmel. C. helvola. fp 90. Marrini 1. 8.30. f. 326. 27. Lister Conch. 691 . f. 38. $18 \mathrm{Si}$.

27914 C. Stercorerie. Det Hochrücken. Gmel. íp.6. Martini 1. t. 38 . fig. 332 6 St.

280 is C. Ligare. Die bebandere Porceltane. Gmel. C. Stercoraria fp. 6. I St.

28116 C. Crenare Die gekerbte Porcellane. Gmel. C caurica. Ip 83. Martini I. 8. 29. f. 303.3 St.

28217 C. Lentigo. Die Sommerfprofte Gmel. C caurica Sp. 83. I St.

283 is C. Annulus. Die Ring Porcellane. Gme!. C. fp. 82. Martini 1. 1.24 6.239. $24018 \mathrm{~S}$ :.

284 19 C. Manete. Die Münze Gmel.C. Tp.8t. Martini I 131 f. 339.340 .4 St. 
285/20 C. Numisma. Die Pfennings Poreella. ne Gmel. C. monetz fp. 81. Martini I. f. $337.338 .5 \mathrm{St}$

28621 C Vorioloja. Die Blatter. Porcellane. Gmel, C nucleas. Ip. 95. Mart. 1.t. 29. 318. Kuort 4. 2. 17.6. 7.6 St.

28721 * C. Lima. Die Feile. Gral. C. \{g. 95. var. 3 St.

$28822 \mathrm{C}$ Oniscus Der Kellerwurm. Gmel. C. 'p. 93 var. 3 St.

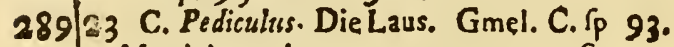
Martini I tab. 29:310.31!. 2 St.

29024 C. Pulex. Der Floh. $2 \mathrm{Se}$.

2. Regulares. Ausgewachlene.

$29 \mathrm{~J} 25 \mathrm{C}$ Caralicxlnea. Die dünnfchaligre Por. cellane. Gmel 'p. 14. fragilis. Guatei. tab. 16. Gg. G. G. $6 \mathrm{Sr}$.

29226 C. Cruentata. Die blutrothe Porcellane. Gmel. C. fqualioa. 'p. Ior. Das Emmerlings. Ey. Gualti. tab. I6. fg. R. Mastini I. t.25. E.250. 5 I,

\section{Lade 13.}

29327 C. Onea Gmel. C. rigiris. Ip. 44 Mart. I t. 24. f. 234. ISt.

29428 C. Tigris. Die Tieger - Purcellane. Gmel. C. Ip 44. Mart. I. 1. 24. f- 232. 33. 14 5r.

255129 C. Pardus. Die Parder - Parcellane. Gmel. C. SP. 44. Mart, I. t. 24. .. 235. $6 \mathrm{St}$.

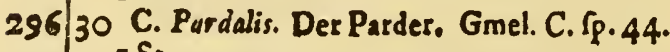
5 St.

2973 I C. Lynx. Die Lux - Porcellane. Gmel C. IP. 48. Marcini I. t.23. 230.3I. II Sa. 
298132 C. Virellus. Die weifsgeffeckre oder Salzkorner Porcellane. Gmel. C. guttaca. rp 15. Mast. 1.t. 23. f. 228. 29. 13 St.

299 a. Martini r. t. 25. f. 252 . I St.

$3 \mathrm{Cc} 33$ C. Ocitlata, Die geäugelte Porcellane. Gmel. \{p. 9x. Mziruini I. 1. 31 8g. 333. 34. 4 Sr.

30134 C. Coeca. Die blinde Porefllenz. Gmet. Cypr poraria Fp. 92. B Mattinil. s. 24. f. 237 . I St.

Lade 14.

302 35 C. Ziczack. Dic Ziczack-Porcellabz. Gmel.C TP. 54. Mart, 1. E. 23. โ. 226. 27. 3 St.

30336 C. Globulus. Die Kugel- Porcellane. Gmel. C Sp. 99. Martini 1. 6. 24. f. 242. Kwors 6. t.2 I. f. 7. ISt.

Cypraca. Die Porcellane. * Cyhndracear Walzenförmige.

3. Mueronala. Zugefpitzle.

30437 C. Tefudinarta. Die Scbildpart. Porsellane. Gmel. C f. 5. Martini r. c. 27. f. 27.8 .72 .3 St.

30538 C. Meleagris. Die Argus - Porcellane. Gmel. C. Ip. 1. exantbema. Matr. 1. t. 26. f. 257.258 . Chemn so.t 845 . f.1343. 3 $2 \mathrm{St}_{\text {. }}$

- - Knorr 1. cab. 5 sig. 3. $2 \mathrm{St}$

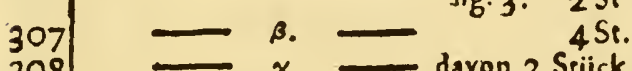

308 r. davon 2 Stiick ab.

309 3, Knorr 2. 8 St fig. 2. 1 St. 


\section{$\operatorname{sen} 2$}

$310 / 39$ C. Mappa. Die Landikarten. Porseilane. Gmel C. Sp 2. Martini 1. t. 25. 1.245. 4.6. Favanue $2 a b .29$ Gg. 4.3. 5 St.

\section{Lade 15 .}

$3 r$ go C. Arabica. Die arabilthe Buchitaben. Porcellane. Gmel. C. P. 3.

312 - $\square$ Mattini.T. t.23. f. $3282920 \mathrm{Si}$.

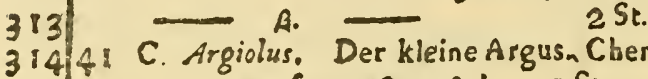
314 41 C. Argiolus. Der kleine Argus Cbemn. \% 44. . 1338 . a \& b 3 St.

315 f22 C. Argus. Der ächte Argus Gmel.

C Sp. 4. Martini \& 28.285.86. Chema. 10.t. $145 \cdot$ f. 1344.45 .8 St.

316,83 C. Glauca. Der Maulwurf. Gmel. C plum baa. \{p. 17 dickfchalige. Martuni $t$. t. $26 . f_{0} 256$ St.

31744 C. Zebra. Der Kapfobe Efel. Gmel. Sp. 17. plumbea dǔnníchalig. Martini $x_{\text {. }}$ t. 26. f. 256 . 8 St.

31845 C. Carneola. Die rothe Fuchs-Procellane. Gmel, 'p 7. Marsini I t. 28. 1287. 28812 St.

31946 C. Talpa. Dis rothe Maus. Gmel. Tp 9. Martini I. tab. 27. f. 273. 274. 5 St. 32047 C. Lurida. Die miesfarbigte Porcellane. Gonel. Sp. 11. Mareıni 1. t. 30. 8. 315 . $32,1 \circ 8$ C. Soricine Die Spirzmaus. 2 St. 322 . C. Ifabella. Die ifabelfarbene Porcellane. Gmel. [p.49. Martini 1. 1. 27. 8. 275. Knorr. 4. 8. 9. fig. 5.4 St

323150. C. Pyrum. Die birnförmige Porcellane. Gmel 6p. 59. Martini 1. t. 26. f. 267. 268 Knorr. 2 :5. .5.5 II St

32451 C. Undofa. Die gewellte Porcellane. Gmel. Amerhyitea, 'P. I0. Martini I. - 25 f. $247-249$. Knort. Vergn. S. ¿. $28.6 .5 \mathrm{SSt}$. 
$325 / 52 . C$. Amethyftea. Die smet? Porcellane, wie No. 58. nur abgefchils. fen. I St.

32653 C. Crenulare.. Die angefsbnittene PorPorcellane. ISt.

2. Umbilizatce. Genabeice 32754 C. Rubiginofa Die ronfarbene Porcel.

328 55 C. Ixterrupta. Die Türkspapier Pore cellane. I.St.

32956 C. Petechialis. Die.Fleckeo-Porcellaue. Gmel. C. Sanguinoleaca. Sp. 38. Mart. I. 8. 26. r. 265.266 . Si

33057 C. Stolida. Die Oliven. Porcellane. Gmel. Ip. 89. Martini I. t.28. 6.292.93. $10 \mathrm{St}$.

\section{Lade 16.}

33158 C. Olive. Der Olivenkero. 4 Se

33259 C. Cornus. Die Kornele. Griel. Cyoz. Scolida. 'p. 89. B. Martioi 1. tab. 28. f. 294.95 . ISt.

33360 C. Draco. Die Drachen. Porcellane. Gmel. C. Rubiginofa. \{́. 105. Mart. I. t. 29 f..305. ISt.

33461 C. Quingue fafciaro. Die fünfbandige Porcellane. Gmel. C. Stolida. Tp. 89 Martini r. 28. f. 292.293. 4 St.

33562 C. Morbillofa Die Marern. Porcellane. Gmel. C. caurica. fp. 83. \%. Martini is. 129. 5 303. 7 St.

33663 C. Erofa. Die gebeitzte Porcellane. Gmel. Sp 84. Martini 1. tab. 30. f.320. 328. Knoer, 6. t. 20. f. $4.13 \mathrm{St}$.

33764 C. Cribraria. - Des Goldtropfen. Gnel. Pp.80. Marini I. 8. 31. f. 336 . 6 St.

33865 C.sffllus. Das Efelcheo. Gmol. Sp.56. Mastioi :. t.27. f. 280 II St. 


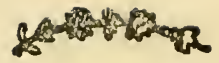

339]66 C. Ovwan Paferis. Das Sperlings.Ey. $6 \mathrm{Sr}$

Casfis, Die Sturmhaube.

* Verce. Aechte.

1. Triengulares. Dreyeckigte.

Lebio Lamellato, mit blasterigter Lippe.

340 : C. Capre-equinum. Der Pferdekopf. Gmel Buccin cosnutum. fp. 11. \& Mar. eini 2. t. 35. \& 362. Liftes 1008. $2 \mathrm{St}$.

341 $2 \mathrm{C}$ Hamata Der Fufrangel. Gmel. Buccín Cosoutum. S. I I. y. Lifter 1009. f. 71. C. $2 \mathrm{St}$.

3423 C. Caput Bovis. Der Oehfenkopf. 3 St. 3434 C Tuberefa. Die knolligte Sturmhaube.

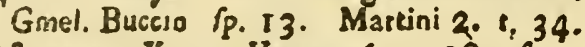
- fo 359. Knose Verga. 6. 1. 18. f. I. 5 St.

5 C. Cornuta. Die gehörnte Sturmhanbe. Grmel. Bucsin cosnutum.

rom.

Mart. 2. t. 33.

f. 348 . 349. 1St.

f. 362. ISt.

$3475^{*}$ C. Pullus. Die Kữer. Sturmhaube. Gmel. Sp. 43. Buccin. Martini 2. t: 41 . 6. 413 . Knorr. Vergn. 6. 2. 22 . f. 5 . s. Si.

$3485^{* *}$ C. Calloss. Die fchwieligte Surm. haube. Gmel. Ip. 43. Buccin. Knorr 6 1.22. א. 6. .

2. Fornicatce. Gewölbte Labio incraffaro, mit vesdikter Lippe.

349 6 C. Rufum. Die sothe Sturmhaube. Ginel. Buccin. If: 12. Martini 2. t. 32. 
f. 34I. Kaors Vergn. 2. t. 9. f. 2. $5 \mathrm{St}$.

7 C. Rufestens. Die bluttolhe Sturmhaube. Gmel. Bucsin. Ip 12. Mart. 2. t. 33. f. 346. 347. Knort Vergn. 2. t. 9. F. $2 \& 4$. t. I. ISt.

8 C. Marmorate. Die gemarmelte Sturm. baube. Gmel. Buecin tuberofum. '́p. 13. Martini 2. C. 38. f. 381.382 . Knort Vergn. 3 t. 10. f. I. 2 . 2 St.

9 C. Elammea. Die geflammte Sturmhaube. Gmel. Buccin. Ip. 14. Martini 2. t. 34. f. 353. 354. Knorr Verg. 4. t. 4. . . $1.9 \mathrm{St}$.

10 C. Tuberculosa Diewatzige Sturmbsobe. Favanne, t. 26. f. 11. 2 St.

355 it C. Plicar um. Die gefaltene Sturmhaube. Gmel. Buccio. rp. 10. Martini 2. t. 37. 379 380. Lifter Conch t. 1002. 6. 67. I St.

356 I2 C. Pileolus. Der polnifche Huth. (davon 3 Stück dünnf(haligte) Gmel. Buccin tefticulum: Sp. I 5: Martiui 2. \&. 37. 375. 376. Koorr Vergo. 4 ? 6. f. ro is St. I.St. a. Martini 2. e. 37. \&. 377. 78 .

13 C. Mitelle Polonica. Die polnifehe Mü. ize. Gmel. Buccin tefficulum; Ip. 15. Martini 2.t. 37. f. $375 \cdot 76$ 2 St.

14 C. Coronara. Die gekrônte Sturm. haube. Gmel. Buccin ceffellatum; P. $_{p} 20$. Mattini 2. : 36. f. $369 \& 37$. \&. 374 . $4 \mathrm{St}$.

35915 C. Pernata. Das tiurkfche Papier. Gmel. Buccin Pennarum. [p. 2 I. Martini 2. t. 36. f. 372. 373. Rumpf, 6.23, 6. C. 
$C a \int S i s . \quad$ Ssurmhaube.
$*$ Spurze. Unechte.

1. Globose. Klugelförmige.

a. Ceudatce. Gefchwänzre.

36016 C. Tyrrbenum. Die tyrrbenifcheSturm. haube. Gmel. Buccin. fp 180 Mart. 10. t. 153 . f. 14611462.2 St.

36117 C. Echinophora. Des Ygel. Gmel. Buccin, fp. 9. Martini 2. 1. 41. 6. 407. Knorr Vergn 1.8 17. f. 1. 4 St.

\section{Lade 17.}

18 C. Brumea. Die braune Srurmhaube. Gmel. Buccin echinophora. fp. 9. 8. Llo ner 1011 . F. 7 1. E. 5 St.

\section{B. Simplices. Einfache.}

36419 C. Glarea. Die Bezoar-Sturmbaube. Cmel. Buccin $\int_{p} 35.13 \mathrm{St}$.

a Eine fehr dünnfshaligre diro. Mart.2. t. 32. 342.43 . ISt.

$36520 \mathrm{C}$. Cancellata. Die gegitrerre Sturm. haube. Gmel. Buccin 'p. $79.2 \mathrm{St}$

36621 C. Guttate. Die weifsgefleckre Sturm. haube. 4 St.

367 \% ${ }_{C \text { Pomam }}$ a St.

36821 * C Pomum. Die apfelförmige Sturmhaube. Gmel Buccin; fp. 4. Martini 2. t. 36. f. 37071 . $6 \mathrm{St}$.

21 * Dünnfchaligte dito. Lifter 1016. f 75 . I St.

36922 C. Areola. Die Gartenbers Sturmhaube. Gmel. Buccin; f. 17. Martini 2. 5. 32. 344. 45. Knorr Vergn. 3. t.8.f. 5 . 22 a Weifte dito ISt. 


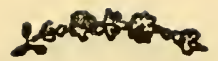

$370 / 23$ C. Malum Die Apricofe. Gmel. Bue cin; fp. 17. B. Lifer 1056. f. 9. 3 St.

37124 C. Globulus. Die kugelfärmige Sturm. haube. Gmel. Buccio gibbum; fp. 24. Martini 2. 1. 34. f. 351 1. 52. Lifter 999. f. $64.2 \mathrm{St}$

37225 C. Cepe. Die Zwiebe!. Gmel. Bncan gibbum; fp. 24. I St

37326 C. Glens. Die Eichel. Gmel. Buscin abbreviatum; ff. 181. Martidi 10.1. 153. 1463.1464 .3 St.

2. Elongas a. Verlângerte.

a. Planiusculas. Gedrlickre.

37427 C. Alec. Dos Bret'piel. Gmel. Buccin arepla ; fp. 17. Martini 2. : 34. f. 355 . 356. Kaorr Vergn. 3 t. 8. 6. 5. 5 St.

\section{Lade 18.}

28 C. Cancellata Die gegieterte Sturm. haube. Gmel. Buccin decurfatum; Ip. 16. Marsini 2 c. 35 f. 360 . 36I. Knort Vergn. 2. I 1U. 6. 3 \& 4 . 5 Sc.

37629 C. Flammoolum. Die Brand-Sturmbau. be. Grnel. Buccin decuffarum; ip 16. Martini 2. 8. $25 \quad 367.368 .25$ S.

377 30 C. Flammifera. Die gefteifee Scurmhaube. Gmel. Buccin Atrigatum; $r_{p} .179$. Martini 10. B. 153 . F. 1457.1458. 3 St.

37831 C. Rugofa. Die runzlichee Sturmhaube. Gmel Buccin plicatuin; $C_{p}$ 10 Martinilo. 8. I 53.f. I459.1460. Lither 10:14. \{. 78 . \& St, 


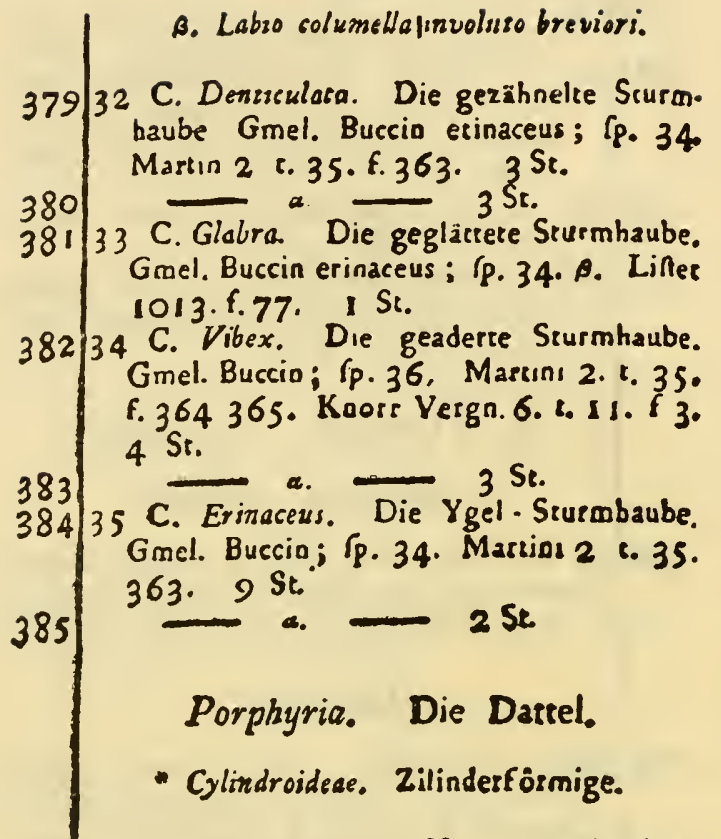

1. Cleverule depreffa, mil gedrucktes Spindel.

386. 5 . Porpbyria. Die Portobello - Dattel. Gmel. Voluta; fp. I6. Martini 2. t. 47. 485. 486. Knorr Vergn. 1. t. 15. 6. \$. 8 St.

3872 P. Fasciara, Die gebandete Portobel. 10. Dattel Gmel. Voluta Ip. 16. \%. I St.

3883 P. Ruffine. Die sothe Datsel. Gmel. Vol IP. 49. v. 2 St.

3894 P. Labradorienfis. Die Schiller - Datcel. Gmel. Volusa Oliva, 'p. 17. Lifes 731. S. 20. 2 St. 
$390 / 5$ P. Serices. Das Seidenzeug. Gmel. V. Oliva. YP. 17. 85. Martioi 2. t.51.f. 559. $561.8 \mathrm{St}$.

3916 P. Miniacea. Das Morgenroth Gmei. V. porphyria. Sp. 16 ค. Martiol 2. t. 45. f. 476477 . 9 St

3927 P. Carulea. Die himmelblaue Dattel. Gmet. V. Oliva Tp. 17. x. Martini 2. t. 48. E 518. Rumpf t.39.6.5. 13 St.

\section{Lade 19.}

39318 P. Variegaza. Die rchäckigte Dartel. Gmel. Voiuta. Ip. 17.8. Martial 2. :45. f. $478.479 .24 \mathrm{St}$. f 480.48 r. Knorr 5. t. 19. E. 4. 5 St.

3959 P. Variabilis. Diefpieleode Dartel. Gonel. V. Oliva. p. 17. 7 St.

396 Io P. Retículata. Die Netz-Dattel. Gmel. V. Oliva. Sp. 17.2. Martini2. t.48.6.512. 533. 9 St.

397 II P. PunEtate. Die geltuppelce Datrel. Gmel. V Oliva $\Upsilon_{p} .174 \mathrm{Si}$.

398 I 2 P Ornata Die gefchmüekte Dattel. Gmel. V. Oliva.fp. 17.2 St.

39913 P. Amoena. Die hübrche Dattel. Gmel. V. Oliva. Sp 17. 3 St.

$40014 P^{P}$ Hepnnce. Die lichegraue Dartel. Gmel. V. Oliva. rp. 17. 2 St.

$40115 \mathrm{P}$. IJabella. Die ifabellfarbene Dattel. Gmel. V. Oliva. P. $_{17}$. 3 St.

40216 P. Aurata. Die goldgelbe Dattel. Gme!. V. Oliva. '́p. 17. I St.

403177 P. Sepulsura Principis. Das Prinzen Beçräbnifz. Gmel. V. Oliva. 'p. I7. \&. Rimp! 5.39 .5 .4 . 
4051 B. - Martini 2. t. 5 t.

f. 563. Koors 5. t 26. f. 4. 4 St. $406 \quad \frac{}{480.481}$. Knors 5.t. 19 f. 1. 3 St.

$407-8$ 8. $=4 \mathrm{St}$.

$408-2$ - 2 St.

$40917 * \bar{P}$ 3.

41017 * P. Oliva. Die olivengrüne Dattel. Gmel. Voluza. SP. 17. ९. Martini 2. 2.45. f. 503.504 I $13 \mathrm{St}$.

118 P. Fismbriata. Eine schöne roftfalbigce Dattel mit jem Ssum. I St.

$4 I I 19$ P. Fisciata. Die Band Dattel, Gmel. V. fp. 17. Knorr 3. t. 17. f. 3. 6 St.

$41220 \mathrm{P}$. Vidua. Die ungarifche Wierwe. Gmel. V. \{p. 17. Martini 2. t.45. f. 472 . 473. $17 \mathrm{Sr}$.

$413 \longrightarrow$ a. $\longrightarrow$ ISt.

414 B. ISt.

4152 I P. Cingulata. Die gegŭrtelte Dactel. Gmel. V. Oliva. fp. 17. I St.

$41621 *$ B. Turgida. Die wulftige Dattel. Gmel. V. Oliva fp. 17. I St.

417 22 P. Feneftrata. Die gegitterte Dattel. Gmel. V. Oliva fp. 17. $\beta$. Martini 2. t 47. f. 502 . 2 St.

41823 Quercina. Die Eichenholz - Dattel. Gme!. V. Oliva Sp. [7. Knorr 5. tab. 26. fig. 4. $6 \mathrm{St}$.

fig. 5. I St. Knorr 5. t. 27.

2. Clavicula elevara, mit hervortretender Spindel.

42124 P. Lutaria. Die gefteckte Dattel. Gmel. Volura. utriculus Sp. 19. ". Martini 2.

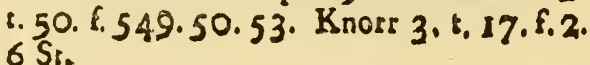


$422 / 24$ * P. Rubicunda. Die söthlishe Dătel. Gmel. V. usriculus $\int_{\text {f. }}$ 19, I St.

\section{Lade 20.}

42325 P. Spicate. Die Kornahre. Grnel V. oliva \{p. 17. Martini 2. 1. 48. £. 509. 107 Se.

42426 P. Grifeola. Die gräuliche Datrel. 3 St. 42527 P. Undulata. Die wellenförmige Dattel. $18 \mathrm{St}$.

42628 P Oculata Die geängelte Dattel. 12St. 42729 P. Dealbria. Die fchneeweirfe Dattel. Knorr 6. t. 34. f. 4 5. $6 \mathrm{St}$.

$42230 \mathrm{P}$ Turbinasa. Die gewirbelte Datkel. Gmel. fp. 22. V. nivea. Martini 2.t. 50. f. 558.4 St.

43031 P. Conoidea. Die kegelförmige Dattel. 1 St.

32 P. Ispida. Die blaumündige Datcel. Gmel Voluta fp. 23 Martini 2. e. 49. f. $524,25.30$. Knore 3, t. 19. 8. 3 . $21 \mathrm{St}$.

f. 535.3 St.

a. - Mart. 2. 1. 49.

Martini 2. 5.49

f. 522235 St.

43633 P. Mice. Die blaugeffeckle Dattel ${ }^{3} \mathrm{Gmel}$. Ip 23. V. Ispidula. 23 Sr.

437 f. $527-\alpha . \quad$ Martini 2. 1. 49 $438 \quad$ B. $327-529$.

439 I4 $_{44}$ P Ametbyfsina. Die amethuftarbene Datrel. Gmel, Yp. 23. V. isfpidula \&. Mar. tini 2. c. 46. f. 4.91 I. 492 . Koosr Vergn. 2. 5. $10.1677^{\circ} 145 t$. 
441/35 P. Tigris. Die Tiger-Datel. St. 44236 P. Palencea. Die itrohfabene Dattel. 44337 P. Pepyracea. Die buntpapierne Datrel. $44438 \mathrm{P}$. Vellus.aureum. Das goldne Vliefz. Gmel. V. Oliva Ip. 17. se. Martini 2. 1. 46. f. $4903 \mathrm{Se}$.

44639 P. Cafra. Die graublaue Datrel. Gmel, Voluca hiatula. \{p. 20. r. Martini 2.t. 50. f. 555 . Lifter t.729. f. 17. 2 St.

44740 P. Stapbylea. Die gelbe Dattel. Gmel. V. Oliva fp. 17. a. Chemn. 10. 1. 147. f. 1381. Lifter t.729. f. 17.18. 2 St.

44841 P. Cornea. Die borafarbeoe Dartel. I $\mathrm{Sr}$.

44942 P. Umbrofa. Die Liche - und ScharcenDattel. Gmel. Voluta ispidula \& $\{\mathrm{p}, 23$. Mattini 2. r. 49. f. 537 . Knort I. T. Is. f. 7.3 St.

Porphyria. Die Dattel.

** Ventricofa. Bauchigre.

1. Clavicula elevata, mis bervortretender Spindel.

450 43 P Aracbroidea. Die Spinneweben. Date tel. Gmel. V. Oliva $\pi$, fp. 17. Martioi 2 เ. 48. E. 509. Jo. ISt.

451
452
44 P. Listerata.

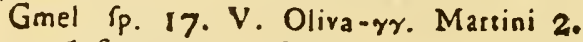
c. $46 . f_{488}$. I $4 \mathrm{St}$

45345 P. Fulgurator. Die Blizz. Datcel. Gmel. Sp. 17. V. Oliva .. Marsini 2.t. SI. f. 562. I4 St. 


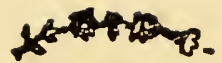

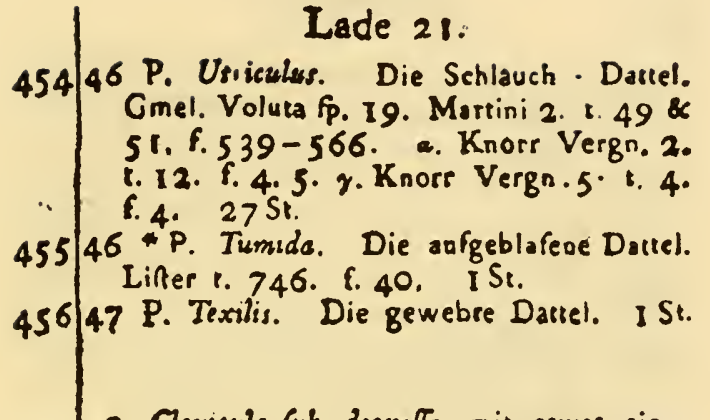

2. Clavicula $\int u b$ - deprede, mit exwas ein. gedruckter Spindel.

457 48 P. Urceus. Der Krug. Gmel. V. Oliva fp. 17. . Mattini 10. t. 147. f. 1367. Kammerer pag. 65. cab.4.f.I.2. $5 \mathrm{St}$.

459.50 P. Bulboja. Die knolligre Dartel. Gmel. fp. 17 . Oliva s. Martini 2. 1. 47. f. 507. 508. $2 \mathrm{St}$.

$460 \mid 51$ P. Tuberofe Die kanehlfarbene Dattel. Kammerer 6. 3. fig.7.8. 3 St.

46152 P. Ziczac. Die Ziczac Dattel. 4 St.

46253 P. Coffec. Die Kaffeebohne. Gmel. fp. 24. V. carneolus. Martiai 2. C. 46. f. 495.4 St.

\section{Cucullus. Die Tute.}

- Pyriformes. Biraformige.

1. Coronate. Gekrönte.

463 1 C. Imperialis. Die Kayferkrone. Gmel. fp. 2. Martini Theil Il. t. 62. fig.69092. Knors Verga. Th. 11. rab. IL. Gg.2. $14 \mathrm{St}$. 
$464 / 2$ C. Corona-Ducalis. Die Herzogs.

Krone. Gmel Conus imperialis. 'p. 2 y. Martini 2 t.62 1.693 .1 St.

4652 C. Regius. Die Känigskrone. Gmel. CP. 2. \%. Martini 10.t 139. f. 1289. 3 St.

$4663 \mathrm{C}_{\mathrm{C}}$ Corona Civica. Die Bürgerkrone. Martini 2 t. 61. f. 678 ISt.

467 4 C. Marmoreus. Die Herz Tute Gmel. Ip. I. Martini Theil II. tab. 62. fig 685. $19 \mathrm{St}$.

\section{Lade 22.}

469 B. 25 .

470 5 C. Proarcbithalajfus. Der Schout by Nagt Gmel fp 1. C.marmoreus, a. Mar. tini 2 t. 62 f. 686 . B. Knors 1. t. 7. f. 4.12 St

4746 C. Equeftris Der gebandere Schout by Nagt. Gmel. C marmoreus. Ip 1 r. Cbemn. 10.tab. 138. f. 1279.2 Se

4757 C. Torquatus. Der Ritter. G mel C Mar. moreus. 'p. I. $r$. Chemn. 10. t. 138. f. 1279. I Si.

8 C. Turrisus. Das Mauetwerk Gmel. C. coronatus. fp. 39. \$ Chemn 10 t. 139. f. 1286-1288. 3 St.

4789 C. Coronalis. Die Kranz-Tute. Gmel. fp. 39. I St.

470 a $\longrightarrow$ ISt.

47910 C. Araneofus Die Netz-Tute. Gmel. C. marmoreus. (P. I. 8, Chemn, 10.1.1 39.
f. $1292.3 \mathrm{St}$. 


\section{L2.}

480|11 C. Aracbroideus. Die Spinnweben-Tute. Gmel. fp 34. Knoer Vergn. 6. t. 4 . fig. 4 Martial 2. 1.6 I. fg. 676.4 St. $48112 \mathrm{C}$. Trapezium. Die Würfel. Tute. Lifter 773. f. I9. I St.

482 1 3 C. Spurius. Der BafatrAdmisal. Knore 6. t. 1. fig. 2.27 St.

48314 ! C. Geograpbicus. Die Eylands. Tute. Gmel, C. Infularis. Ip. 38 3 St. wovon ein vorzïglich fchön und felten. f. 683.8 St.

485 is !! C. Petreus. Der Srein - Admiral. Gmel. C. amiralis coronatus. fi. 10. b. 2 St.

vorzüglich fehön und felter.
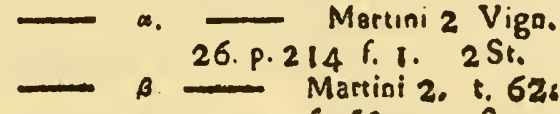

B. - Martioi 2, t. 626 f. $682.4 \mathrm{se}$.

- x. Knors 5. E. 25. fig 3 . $1 \mathrm{I}$ St.

$48915{ }^{*}$ C. Bitiofus. Diebraungelbe Tute. Gmel. C Nercus muscarum. fg 23. 8. Chemn 10. t 139. f 1294. I St.

49016 C. Monacbos. Dar Mönch. Gmel C. ru. ficus Cp 18. B. Martini 2. 1. 63. f 694. Knorr 4 t. 13. f. 3. $12 \mathrm{St}$.

49117 C. Meningeus. Die blutigg Tute. Gmel. C zeylanicus. Yp. 41 Chemo 10. t. 142. f. 1318.5 St.

49218 C. Fernanibucinus. Die bunte Holz.Tute. Martiai 2. t. 57 f $632 \quad 4$ St.

493 I 9 C. Virgo. Die Jungfer. Gmel. C. Sp. 5. 3 Martini 2.2.63 (701-2. 3. Knoss 4 . 1.16.6. 5 St. 


\section{Lade 23.}

49420 C. Arenofus. Die fandige Tiste. Gmel. C. \{p.23 Martini 2. 8.63. f.696. 2 St.

49521 C. Stercus - musearum. Det Fliegen. dreck. Gmel. C. $\{$ p.23 y. Martini 2.t.63. f. 697. Rumpf t. 33. F. AA. IOSt.

49622 ! C. Grenulatus. Die granulirte Tute. Gmel. C. IP 52. I St.

49723 C. PunEtulatus. Det Mŭckendreck. Gmel. C. Ip. 23. B. C. Seereus musearum. Matt 2. t. 63 f. 698.699 .2 St.

49824 C. Granulofus. Die Gänfeheut-Tute. Gmel. Ip. 24. C. varius $\beta$. Martini IO. tab. 138 fig 1284 . 9 St.

49924 * C. Annularis. Die getingelte Tute. Gmel. Ip. 24. C. varius. I St

$500 / 25$ C. Radula. Die Reibe. Gmel. 'P. 24. C. varius 2 St.

50I 26 C. Cenaliculatus. Die gerillte Iute. 5 St.

50227 C. Landoideus. Die Winkel - Tute. 2 St.

50328 C. Folliculus. Die Schore. I St.

2. Simplices. Ungekrôhnte.

50429 C Betulinus. Die Butterwecks - Tute, Gmel. C. Tp. 20. Martini 2.t. 60 f. 665 . Knorr 2. 1. It. f $3.12 \mathrm{St}$. eine von aufTerordentlicher Gröđe. 505: 50 C. Tigris Die Tieger-Katze. Gmel. C Sp. 20 B. 2 St.

s06 31 C. Fraxineus. Die graublaue Tute. Gmel.rp. Is. Chemn. 10. เ. 138 \&. 1277. 1278 i St.

507132 C. Glaucus. Die ciperfehe Katze. Gmel. 507132 C. Fp. I5.Chemn. IO. t. I38.f. 1277.78 2 Si.

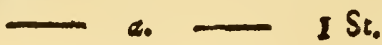




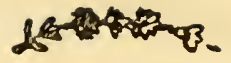

509/33 C. Pavimensum. Der italienifthe Es. trich. Gmel Tp. 5. C. Virgo. Martini 2. tab. 59 8. 653.654 . Knorr 2. t. 12. f. 3. $12 \mathrm{Ss}$.

51034 C Lacteus. Die milchweiffe Tute. Gmel. Ip. 75. C. glaucus. Mastini 2. 1. $6 \mathrm{I}$. 6. 673 . 5 St.

Sil 35 C. Quadratus. Die Rauten. Tute. Gmel. PP. 27. C. leoninus. p. Chemn, rO. 8. 140. f. 1300 . I St.

51.2 36 C. Quadrazulus. Die Blumen. Tute. Gmel. Tp. 15. C. glaucus. Martini 2. เ. 61. 5.670 .671 . 6St.

\section{Lade 24.}

51337 C. Syriacus. Die fyrifche Schrif. Gmet. Pp. 67. C. Spurius. Martini 2 t. 56. f.627. Krors 3. เ. 18. f. 5. .. 9. f. 6. t. 22. f. 3. 9 St.

Ko. Kort 3. 26.

51538 C. Ferugineus. Die roftige Ture. Gmel. rp. 67. C. Reurius. var. Martini 2. t. 56. f. $626-6282$ Se.

51639 C. Listeratus. Die Buehtraben - Tute. Gmel. Sp.3. Martini 2. t. 60. f. 668. $23 \mathrm{St}$.

517 fig. 3. a. Knorr I. 8. 16. 51840 C. Byffinus. Die arabifche Tute, Gmel. IP. 3. C. likteratus B. Martini 2. t. 60. f. 669 . 4 5t.

51941 C. Pardus. Der Parder. Gmel. SP. 3. C. litteratus. Máartini 2. Ł. 60. f. 667. Knorr I. I. 16. ؟. 3 . 3 St.

52042 C. Leopardus. Der Leopard. Gmol. fp. 3. C. litteratus, Martini 2. 4. 60. f. 666.5 St. 


\section{Lacie 25.}

52143 C. Sphinx. Die chinefirche Baftard-Tute. Gmel. C geruatuus. f́. 14. Mattni 2. r. 56. f.624.625. Knorr 3. t. I. f. I. $13 \mathrm{St}$.

52244 !! C. Papilio. Der Papillions- Flūgel. Gmel. C. genuanus Papilio. Sp. I4. \&. Marrini 2. t. 56. f. 623. Argenv, t. 12. f. V. 3 St.

52345 !! C. Genuinus. Dic àcbre chinefifcbe Tute. Gmel. rp. 68. C. vexillum. Lifter 763. f. 12. A. Chemn. 10. $\therefore$ 144. A. f. $\mathrm{m}$. n. die Flagge der dreyzehn vereinjgren Staaten. I Sr.

524 46 C. Ebrous Die Bauern-Mufrk. Gmel. Ip. 22. Martini 2.t.56. 6.617. Knorr 5. 2. 6. f. 2 . II St.

52547 C. Chaldaus. Die 3 St. C. Chaldaus. Die Chwarze Ture. Gmel.
C. princeps. Tp. 9. Matrini 2. 2. 63. f. 699.700. Knorr 3. t. 4. f. $2 . \quad 5$ St. 52648 C. Figulinus. Die Eichenholz Turs Gmel. If. 21. Knorr 5. t. 25. fig. 2 4 Se.

$527-\alpha_{0}-7 \mathrm{St}$.

$528-\beta$. $-3 \mathrm{St}$.

$529 \quad !-\%$ I St.

S36 49 C. Buxezs. Die Buxbaumbolz-Tute. Gmel. Ip 21 . Knorr 3. t. 11. fig. 2. Martini 2.e 59. f.656. $658 . \quad 5 \mathrm{St}$

53150 C. Cardinalis. Die Cardinals. Tute. Gmel. C. aurifiacus. fp.56. Chemn.10. t. 144. A. f. I. 2 St.

532 II C. Virgo. Die Menonilten. Ture. Gme: P. 5 Martiai 2. t. 53. $\{585$. Koorr 2. t. 24 f. 4 \& 3.t. 22 . f. 1.6 St.

53352 C. Gualterianus Die gualterifche Tute. C. Spurius. Gmel. fp. 67. Gualkiv, 21. fig. F. I St. 


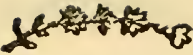

53453 C. Cinamomens. Die kanehlfarbene Tuste. Gmel. C. eapisaneus. Sp. 6. ro. Martini 2. i. 57. f. $680.68 \%$ i St.

53554 C. Crnonicus. Der Domherren-Mantel. Gmel. C. vexillum. fp 68. Martini 2. t. 57. 6.629. Koorr 3. r. 1.6.3. 4 Se.

33655 .C. Miles. Der Soldar. Gmel. 4.8. Martini 2. t. 59: f. 663.664. Knots Y. 8. I5. f. $4 \& 3$. r. I. f. 3. $13 \mathrm{St}$.

\section{Lade 26.}

$\$ 3756$ C. Capitaneus. De: Haupemann. Gmel. Sp. 6. Martunt 2. t. 59. f. $660-662$. Knorr I. t. 1 5. f.3. 9 St.

538

539

540

541

542

543

f. 1280 .

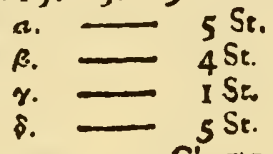

- a. $5 \mathrm{St}$.

$=\beta_{0 .}={ }_{1}^{4} \mathrm{St}_{\mathrm{S}}$

$=\delta_{5}={ }_{5} \mathrm{St}$.

3. $\quad{ }_{1}^{5} \mathrm{St}_{\mathrm{St}}$.

$544 \longrightarrow 2 \mathrm{St}$.

54557 ! C. Arcbitbalafjus India. Der wertindifche Admiral. Gmel. C. Thonix. Sp. 70. Cinemn. 10. t. 138. f. 1282.83 . roSt, 54658 C. Medufa. Der Medureukopf. Gmel. C. betulinus. medura. '́p. 20. B. Mart. 2. t. 61. 6. 675. Chenun. 10. t. 142. f. 132 I. ISt.

54759 C. Acbotinus. Die Agat . Tute. Gmel. C. lenninus. If 27. 3 . Martini 2. c. 55 . f. 605.636 . $\$ 3$ St.

$54859^{*} \mathrm{C}$. Baratbrum. Die weimundige Tute. Gmel C. leoniaus. SP. 27. Mastini 2. t. 55. f.607. I St.

54960 C. Theobroma. Die zerrifene Neez. Tute, Gmel. C. zeylanicus P. $_{\text {p. }} 4 I$. Chemn, JO. t, 142. f. $1318.2 \mathrm{Sc}$ 
Lade 27.

55061 C. Flammeus. Die flammigte Tute. Gmel. P. 27. C. leouinus, Martini 2. t. 55 . f.606.607. ISt.

551 62 C. Leoninus. Die kliminende LôweoTute. Gmel. ip 10. Ammiralis americanus. Martini 2. 4. 57. f. 640. 2 St.

$55^{2} \sigma_{3}$ C. Ifabelle. Die ifabelfarbene Tute. I St.

55363 * C. Bifasciasus. Die zweybandige Tate. I S.

554 ! 64 C Cedo Nulli. Die Cedo.Nalli.Tute. 3 St.

$55565 \mathrm{C}$. Annulus Die Ring-Tate ISt.

55666 C. Pulverulentus. Die geftippelte Tute. Gmel..P.57. C. magas. Martini t 58 . f. 641. knorr 6. \& 16. 6.5. \& t. I. E. 34 St.

55767. C. Cereolus Die Wachskerze. Gmel. rp. 4. C. gederalis B. Chemb, I0. t. 140. f. 1301 . I St.

55868 C. Orleanus. Die orange Tute. Gmel. IP. 6. C. capitaneus 8. Martini 10. t.144. f. 1298. Koorr 3. t. 18. f. 3. 6St.

55969 C. Filofus. Die umlponnene Tute. Favanne I5. fig. C. I St.

$56070 \mathrm{C}$. Ornatus. Die gefchmückte Tute. Gmel. rp. 4. C. generalis $\beta$. Marr. ta t. 140. f. $130 \mathrm{~T}-1303$. 4 St.

561 78 C. Dux. Der Feldmarfchall. Gmel. rp.4. C. generalis. Martini 2. t. 45 . f.648. I St.

56272 C. Generalis. Der General. Gmel. IP. 4 . Martini 2. 8. 58 . f. 646. Knose 1. t. 7. f. 3 2.t.5 f. 2 \& 3.t. I7. f. 45 \& t I8. f. 3. 4. II St. 
$564 / 73$ C. Locumtenens. Der Lieurenaot. Gmel. fp. 4. C. generalis. Martini 2. t, 58. f 645.2 St.

56574 C. Ebstrneus. Die elfenbeinetne Tute. Gmel. Tp. 48. C.ochrolzkcus. Mark. 3. t. 52 f. 573.2 St.

56675 C. Amadis. Die Amadis. Tute. Gmel. Sp. 32. Chemn. 10. t. 142. f. 1322. Knors 6.t. 5. . 3.9 St.

56776 C. Vicarius. Der Vice.Admiral. Gmel. Sp. IO Ammiralis Americanus b. Mart.2. t. 57. f.638.639. Knorr 5.t. 24. f. 4 . 5 St.

$568 \quad \alpha_{0}-2$ St.

56977 C. St. Thomee. Die St Thomas - Tute. Gmel.1p.70. Chema 10.t.138. f.138 T. D.2. Mart. 2.t.53. . 590.2 St.

57078 C. Pulcbellus. Die fchöne Tute. Fa. vanne tab. 79. fig. L. 2 St.

57I! 79 C. Ammiralis. Die Admirals. Tute. Gmel. C. Ip. 10. occidentalis c. Martini 2. t. 57.6 .635 . St.

572! C. Summus. a. Der Ober - Admiral, mit 3 Bänden. Gmel. fp. II. C. Vicarius. Chemn. 10. t. 141.5 I 307.4 St.

573 ! C. Princeps. B. Der Rang-Admiral, mit 4 Bândeo Gmel. C. ammiralis fum. mus.a. Ip. IO: Favanne.t. I7. f. S. Knorr. T. t 8. f. 2. 2 St.

574 ! C. Coronatus 8. Der gekrönse Admiral, mit 5 Bänden. Gmel. Sp. IO. Cherso. 10. $t$ I 4 1. f. 1308 . 1309. 2 St.

575 ! C. Imperialis * . Der kayferlicbe Admiral, gelblich mit 4 Banden. I St.

576! C. Granulatus 7. Der granulirte Admiral, mit 4 Band a. I St.

577! C. Torquatus \&. Der excsa Admiral, mit 6 Bānden. Favanne. Sab. 17. 58.4 . 2 St. 
$578 /$ C. Equeftris $\xi$. Det Ritter. mit 5 Ban den. Favanne. tab. 17. fig S. 2 ISt

579 ! C. Larvatus9. Der verlarve Admiral. mit 4 fchmalen Banden Gmel $\Gamma_{p}$. IO.e. Martini 2. 1.57. f. 635. a Favande. iab. 17. fig. S. 3. I St.

580 C. Dubiratus n. Der After-Admiral, I St.

581 80 C Crenulasus. Die gerillite Tute. 2 St. 58281 C. Tornarus. Die gedrechfelte Tute. 2 St.

58382 C. Minutus. Die kleiae Ture. Gmel, Ip. 16. C. monachus. Martinl 2 t 55 . f 612 a. $8 \mathrm{St}$.

$58483 \mathrm{C}$ Nubecula. Die gewälkre Ture. Gmel. fp. 66. Marsıni 2. t. 56.f 622. 7 St.

585 84 C. Paruus. Die kleine Tute. Gmel. Ip. 66 . ISt.

58685 C. Nubilus. Die dunkle Tute. Gmel, 'p. 27. C. leoninus 8. Mastioi 2. 8.55. f. 6106 St.

58786 C. Pellucidus. Die durchfichtige Tute. 2 St.

588187 C. Clavarus. Die afchgrave Tute. Gmel. 5p. 18. C. rufticus a. Mastini2. c. 52 f. 578 . 2. Si.

\section{Lade 28.}

58988 C. Cinereus. Der Afchen Păfet. Gmel. fp. 18. C. rufticus. Martini 2. 1. 52. f. 578.4 St.

89 C. Cinerarius, Der Afchentopf. Knorr 3. c. 16 fig. 3 grawe Monnik. I St.

59190 C. Spętrum. Das Gefpentt, Gmel. fp. 62. Martini 2. c. 53 . r. $581-583$. Knoss 2. t. 8. f. 4. 9 St.

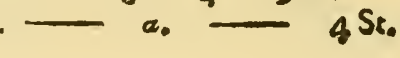


$593 / 91$ C. Lineazus Die linirze Tute. Gmel. fp. 62. C. SpeQtrum. Chemn.10.1.140. f. 1304 Knorr 2. 1. 8. f. 4.3 St.

59491 * C. Albeolus. Die weiffe Kerze. Gmel. ip 44. C terebellum. Martini 2. t. 52.
\&.577. I Se.

59592 C. Cbinenfis. Die chinefilthe Scbrift.

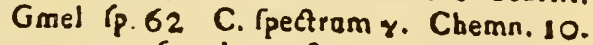
t.144. a.f.g.h. 3 Se.

59692 *C. Ansillarum. Der englifche Admi. 821. Gmel. '́p. 47. C. latus. Koorr 3. 16. f. $5.7 \mathrm{St}$

59793 C. Carota. Die sothe Rübe. Grael. Ip. 62. C. Spectrum. Martini 2. t. 52. f. $58 \mathrm{r}$. I St

59893 *!! C. Aurifacus. Der Orange - Admiral. Gmel. ŕp.56. Knorr 5. .. 24. f. 1. I St.

59994 !C. Venuffus. Die liebliche Tute. I St. Eine böchtreltrae weine dünnfchaligte mir blafsrotben Wolken bezeich. nete Tute zum Gefchleche des Amadis, doch etwas bâuchige.

60095 C. Daucus. Die gelbe Rübe, orange farbigr. I St.

60196 C. Sponfa. Die Braut. $3 \mathrm{St}$.

60296 * C. Purpureus. Die blutrotbe Tute. Gmel. Sp. 64. C. tulipa $\beta$. I St.

60397 C. Metcalor. Der Kaufmana. Gmel. fp. 19. Martini 2. t. 56. f.620. Knors 2. t. I. f. $4.5 \mathrm{St}$.

60498 C. Aurelius. Das goldne Netz. Gmel. fp. 19. Martini 2. t. 56. f. 62 I. Knorr 2. t. I. f. 43 St.

60599 C. Mille punEtatns. Die taufendfach punctirte Tute. Gmel. Ip. 36. C. leuco. stictus s. Chemn, 10. 1. I\$30. 6. 1305. 6 St. 


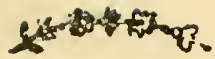

$608 / 100$ C. Caffer. Die zimmetbraune Ture. Gmel. fp. 31. C. coffez. Martini 2. :56. F.618. $2 \mathrm{St}$

607101 C. Anosymms, Die samedlofe Tute. $2 \mathrm{St}$.

608 102 C. Novem firiatus. Die gmal gefreifre

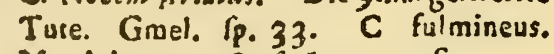
Martini 2 t. 58 f.644. 2 St.

609103 C. Torwains. Die ausgedrechfelte Tute. ISt.

6:0 804 C Faspideus. Die körnigıe Tute. Gmel. Sp, 28. Martini 2. e. 55. f.612. St.

$611 \log$ C. Circundutfus. Die weiffe Schnur. 2 Se.

6I2:105 C. Politus. Die geglätrete Tute. I Se.

613106 C. Circumvolutus. Die graugrüie Tute. I St

6r4 107 C. Saphirus. Der Saphir. ISt.

615108 C. Glaucus. Die bläuliche Tute Gmel. Sp. 17. C. minimus. Martini 2. t. 55. $66133 \mathrm{St}$.

616 109 C. Cefsurs. Die weifzblaue Ture. Gmel. fp. 25 C. achatious. Martini 2. 8. 52. f. 580 St.

617 110 C. Crucifer. Die Kreuzrtâgerin. Gmel. [p. 27. C. leonimus $\beta_{0}$ Martioi 2. t. 55. f.605. 2 St.

6181370 "C. Camelinus. Die kaneblfatbene Ture. I St.

6:9|1 II C. Carsis anguina. Die Schlangenhaut. Gmel. \{p.27. C. leoninus, Martiai 2. t. 5.5. 6.605. 5 St.

620 I 12 C. Maculofus. Die bunte Ture. Gmel. fP. 29. C. nebulofus ... Kaoss S. t. 18. Sig. 4 Knort 3. t. 16. E. 3. 8 St.

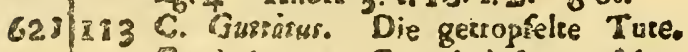
Gmel. fp. 29. C. neluslofus $\beta$. Mar. tini 2. 8.55.8.613.614. ISt. 
$623 \mid 114$ C. Ventricofus. Die bauchigre Ture. Gmel, if 25. C. achatinus. Chemn. 10. c. $142.81317 .2 \mathrm{St}$.

Cucullus. Die Tute.

- Cylindraced. Walzenformige:

I. Coarctase.

624115 C. Magus. Der Zauberer. Gmel. Sp. $625 \quad$ 57. Martioi 2. .5 58. . $64 \mathrm{I} .25 \mathrm{St}$ $626-\beta-1$ St.

627116 C. Arenatus. Die Staub. Tute Gmel. Sp. 23. C Atescus mulcarum. Marcini 2 r. $64: 711.71295 \mathrm{Se}$

$628116{ }^{\circ}$ C. Sabilla Die rothe Sand - Twre. Gmel. 1p. 23. C. Atercus mufcarum. Martini 2 t. 64. f. 7 I $3.3 \mathrm{Se}$

629117 G. Nobilis. Die buote Madam. Gmel. 1.. :3. Mart. 2 + 62 f. 689. Chema. 10.: 141.8 .13124 St.

630 II C Aureus. Das goldne Zeug. Ginel. Ip 60. C au seus is St.

631 Gmel. (P. 60. \%) Mart. 2. 2 54.f.60r. 2 St.

632 I19 C Ausiger. Die verbrelimte Tute. Ginel. ip. 59. C rextilc. Martini 2. t. 54.85496 Sc.

\section{Lade 29.}

633120 C. Gluria maris. Die Gloria Maris Iure, 2re Sorte. Gmel. Sp. 59. C. tex. tile. Martini 2. t. 54. f. 598.600. 602. $\beta$ Chemn. $10 . \mathrm{r} 143 . \mathrm{f}_{0} 1326$ a. $2 s \mathrm{St}^{2}$

634121 C. Aurifer. Die vergoldete Tate. Gmel Ip 60. C. aulieus. Korr 2.1 I. E $1-3.8 .3$. t. 19. S. X. 16 Sto

D

635 
$635 \mid 122$ C. Auratus. Diegüldifehe Tute. Gmel. S. 59. C textule. Rnorr 2 t. 8. . 3. 8. 3.t I 8. f. 2. 7 St.

636123 C. Textile. Die Goldlaks Tute Gmel. fp. 59. Martini 2.t. 54. f. 598. To St.

637 \%. Chemn 10. tab.

638124 C. Aulicus. Die bunte Tute. Gmel. fp. 60. Martini 2. 5. 53. f. 592.595 . Knorr 3. t. 19 \& 1.13 St.

639125 C. Gentilis. Die kurzgefpindelte Brunet.Ture. Gmel 6. 60. C. aulicus a. Martini 2.t. 53. . . 591 . 6 St.

\section{Lade 30.}

$640 / 26$ C. Striatus. Das Wolkhorn. Gmel. Sp. 58. Martini 2.t. 64. f. 714-716. Knosr 1.t. 18. f 1. \& 3 t. $12 . \mathrm{f}$ 5.t. $24 . f_{.} \mathrm{I} \cdot \mathrm{I} \cdot 18 \mathrm{St}$.

641127 C. Nuffatella. Die Sand Tute Gmel. fp. 43. Martini 2.t. 5 1. \& 567. Knort 2 . 4. F. $_{7}$ : 9 St.

$642128 \mathrm{C}$. Terebellum. Der Küperbohrer. Gmel. fp. 49. C. laevis. Martini 2. t. 52 f 572 i St.

643129 C. Condidus Der weifte Küperhobrer. I St.

644 I30 ! C. Purpuratus. Der vice OrangeAdmiral. Gmel. fp. 50. C affinis. Mar. tini 2. t. 57. . .571. I St.

645131 C. Cbryfalis. Der gelbe Küperbohres. ISt.

\section{Laxae.}

646132 C. Geographus. Die Agat - Bake. Gmel. Sp. 65. Martini 2.6.64.6.717. Knorr 3.t. 2I. E. 2. IOSt. 
$647 \mid 133$ C. Tulipa. Die Tuipe Gmel fp. 64 . Martini 2. t. 64. 65. f. $718.72 \mathrm{I}$. Knorr 3. t.11.6 4 \& 5.1. 20 . f. 1.2. II St.

648 134 C. Leganum Die purpu Bake Gmel. fp 63. C. bullatus. Knorr 5. : 11.84 . ist.

649135 C. Bulletus. Die orange Ea:ze. Gmel。 5 63. Chemn. BO. t, 142. 8.1315. 1316.2 St.

650136 C. Capisetus. Dex Srockknopf. I St.

\section{Lade $3 \mathbf{I}$.}

Ptorygia. Halbe Fligel-Schnecke.

651 I P. Glabella. Die glatte halbe FlügelSchnceke. Gmel tp. 32 Voluta Glabella. Martini 2. t. 42. f. 429. Knort 4. c. 21. f. $2 \& 3$ \& 4 St.

6522 P. Nebulosa. Die wolkigie halbe FlligelSchoecke. Gmel. f. 32. Martini 434. $435 \cdot 10 \mathrm{St}$

653 P ${ }^{2}-4$ St.

6542 "P Vulgaris. Die kleine gemeine b $F$. Schnecke. Gmel. if 35 Voluta mercsto. ria. Marrini 2. $244 \quad 456457 \quad 2$ St.

6552 *.P. Amana. Die büblcheh. E Schoecke. Gmel. Volua mercatoria fp. 35. Martini 2 t 44.6458 . 2 St.

6563 P Erminea. Die Hermelin halbe FlügelSchnecke. Gmel Voluta faba. Ip. $_{3}$ I. Martini 2 t 42. f. 432 433. Koorr 4 . t. $17 f 62 \mathrm{St}$.

$6573 * \mathrm{p}$ Subrerranea. Die unterlirdifebe b. F. Schnecre Favanne Tab 56 f. J. 1. Mastini 4. 5. 142. 1330.3 St. 
65813 **. Effoffa. Die ausgegiabene halbe Fl. Sclonecke. Favanne Tab. 56 f. J. 1. Mas. rini 4.t. 42 . f. 1330 . 4 St.

6594 P. Prana. Die Pfaume. Ginel. fp. 33. Voluta prunum Martini 2.t. 42. f. 422 . 423. $5 \mathrm{St}$.

6605 P. Perficnla. Die gefâumte b. F. Schnecke. Guvel. fp. 29. Voluta perficula y. Martini 2. $42 . \mathrm{f} 416.2 \mathrm{St}$

6616 P. Craffa. Die doppelt gefaumte h F. Schnocke. Gmel fp. 29. Valuca perficula a. Martial 2 t. 42 . f 42 I. Chemn. 10.4 150 f. 1421 . I St.

66277 P. Folfilis. Die Follil b. F. Schnecke. $8 \mathrm{Sr}$.

$66374 \mathrm{P}$. Turris. Die langgeftreckte h. F. Schnecke. I St.

6648 P. Magellanica. Die magellaniche $h$ F. Schnecke. Grasl. Voluta. fpectabilis $f_{p}$. 142. Chemn 10,t I 48. F I 383.884 . Kammerer 7 6g. !. Knors 4.229 f. I. 2. I $\mathrm{Si}$

5659 . H. Peregrisa. Die gelbe ${ }^{1}$ magellanifche b. F. Schnecie. Gmel. Volura, Spectabi.

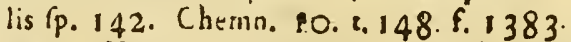
\& 84. Kinot.r 4 t. 29 f. I. 2 . 2 St.

665 a $9 \propto$ ! I P. Fulgura Die Blitzintahlen h. F. Sclinecke. Ginel fp. 106. Voluta, ru. peftris Martini 3. 5. 98. f. 941.8942. St.

666/10 P Ancilla. Die getlammte magellani. fobe h. F. Schnecte Gmel fp. 110. Volusa magelanica Chemn. Tom, X r 148. 1 1383.1384. Knoir 5. 1. 23. f. 2 isc,

667 I I P Volva. Die werlle bi F Schnecke. Gmel. fo 126 . Volura Gluemn. 10.0 148. \%: 138913902 St.

$66812 \mathrm{P}$. Edencula. Die zahalofe b. F. Sebn $5 S t$. 
669|13 P. Nucella. Die Nuls Gmel. \{. 25. Voluta dactylus. Lifter r. 813 f. 23. Chemn 10. ¿. 150 f. 1411.12 .35 . 67014 '' Pyriformis. Die unreife Birne. 2 St, 67115 P. Conordes. Die braungtieckte Birre. Gnel Ip 140. Voluta conus. Cbeann. 10. 8. 150. f. $1415 \cdot 16.2 \mathrm{St}$.

Morun. Die Maulbeere.

672 I M. Purpureum Die purpusfarbene Matb beere. Gmel. Sp. 18. Strombas onifus. Maıtuni 2. c. 34. f. $357 \cdot 358$. Kacir 4. ז. 12. 8. 4. 6 St,

\section{Galeodes. Der Helin.}

6731 G. Melongena. Das Betrzeng. Gmel. Murex Melongena. Ip. 50. 674 f. 393.397 .3 Se Marrini 2. 4. 39.

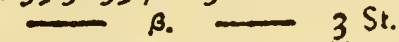

676 \% — Knors I. C. I\%. f $55 \mathrm{St}$.

677 8. Martini 2, t. 39. 6. 391. 9 Si.

\section{Lade 32.}

678 E. - Chemn.10.2.164 f. 1568.7 St.

6792 G. Tuberofa. Der knolligre Helm. Gmel. Murex hippocaftanurg. Ip 48. 3 . Marciai 3 i 100. . $956 . \mathrm{A}$ : Sc。

680/3 G. Mespilus. Die Mispel. 2 St. 


\section{(4-2)}

Thais. Thais.

I. Cleviczla deprefa. Mit kurzer

$$
\text { Spindel. }
$$

68 I I T. Lena. Die Kuplerin. Gmel. Tp. 44. Murex fucus. Martini 3. .. 100. \&. 959. 960. 7 St.

6822 T. Meretricile. Das Fseudenmädchen, Gmel. Tr. 44. Murex fucus. Martini 3. e. 100 6. 961.962 . I St.

2. Clavicula elevare. Mit hervorter. tender Spiadel.

6833 T. Pesula. Der Weiremund. Gmel Buc. cinuns pasulum SP S1. Martini 758759. Knore 6 t. 24 f. 1. 7 St.

6844 T Perfica. Die Schürtruft. Gmel. Buc. cinum perficum. Sp. 49. Martioi 3. 8. 69 , f $760.6 \mathrm{St}$.

685 5 T Panama Die Panama Gmel. Bucci. num bxmaftoma. f́p. 52 . Chernn, 10 t. 154. f. $1467.68 .5 \mathrm{St}$

686 5 T. Grijea. Der blasgraue Weitemund. Gmel. Buccin. hæmaftoma. Sp. S2. Mart. 3. t. 101 . f. 964.2 St.

687.7 T. Metallica. Das Metal-Horn. Gmel. Buccin hainaftoma. Íp S2. Martini 3.4 10. f. 965.966 .7 St.

$688-2$ St.

$689-$ B. -1 St.

$690-y_{0}-\mathrm{St}$

6918 T. Stellara Das gezakte Metal. Horn. Gmel. Buccin. barmaftoma. Í. 52. Argenv. t. 17 f H. I St.

69219 T. Nebullofa. Das dunkle Metal-Horn. Gmel. Buccin。, bemaßroma, 'p. 52. I St. 
693110 : T. Monoceris. Das Einhorn. Gime:. Buccio. monodon. Í 50 Martini $3.16 \dot{y}_{0}$ f. 761. Chemn. 10.t. I54. F. 1469. 70. Knoss 4. t. 30. ร. 1. 2 Se.

\section{- Drupa. Die unreife Olive.}

I. Columella deprefsa. Mit stoge. deuckser Spindel.

6941 D Morzm. Die Maubeere Gmel: Mus rex De Hordeus. Fp. 43. Martini $3: 101$. f. 972.978 . Knots 1. t. 25. 6. 5. 6. 5 Se.

6952 D. Trabulus. Die Waffernufs. Griel Mus. rex ricinus. 'fp. 41. Rumpf to 24, f. $\Gamma_{0}$ Gualt. t. 28. r. N. 2 St.

$6963 \mathrm{D}$ Rubus rafius. Die Hindbeere Gmel. Murex neritoideus. Martial 3 6. 801 \&. 9769774 St.

6984 D. Rubus ideus. Die weifie Hind'sers. Gmel. Murex hyftrix Ip 46. Marsin: 3. c. 101.f. 974.975. Knort 6 เ 24 f. 7 . 4 S:.

69215 D. Fragum. Die Eidbeere. Gme?. Mussez nodus 'p. 42 vas 1 Se.

7006 D. GroJfularia Die Stachelbecre. Gmel. Murex nefitoideus fp. 43. Mastiai 3.60 102. 978979.2 Sto

2. Columelle elevase. Mit barvor tretender Spindel.

701 7 D Chamemoras. Die fchwarze Maulbeete. Gmel. Murex mancinella. F. 47 Lifer 954. f. 4. Knort 4.t-26 E. 2. 2 St.

7027 D. Botroides. Die Wearraubs, Pavanne Tab. 33. fig. B. 2 St. 
70318 ! D Uve. Die Weinheere. Gmel. Mu. sex mancinella. Sp. 47. Martini 3.t. IOT. 8970. Lifter 0 954.6 5 I St.

7049 D Cornus. Die Kaneel. Kirlche Gmel. Mus ex mancinella. Ip. 47. val. Mattini 3. I. 101. f. 971. 2 St.

706 10 * D. Murricine. Die purpurfarbene Maul. beese. Gmel. Murex Sacellum rp 164. Chemn 10 t. 163. f. 1563.62. ISt. 70710 *D. Glans. Die Eichel. Gmel Murex despe\&us Sp. 74. Chemn. 10 t. 163. f 1598 . I st.

708 II D. Mantinilla. Die weiffe Mauibeere. Grael. Murex macinella. ip. 47. Knors 3 5. 29. Gg. 6. 4 St

70912 D. Trape. Diegrolle Maulbeere. Gmel. Murex Lippocananum Ip 48 B. Martini 3. 8. 100.1 .9575 St.

710 13 D. AEscribs Die dunkle Maulbeete. Gmel. Murex hippocaftabum. ́p. 48. var, $3 \mathrm{St}_{0}$

\section{Lade 33.}

Vasum. Die Wafe.

711 : V. Uma Der Afchentopf. Gmel. Vo*

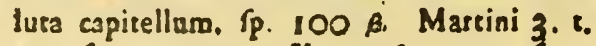
99. \& 949. 950. Kaoss 6. 8. 35. ร. 1. 7 S.

712 2 V. Capitzllum. Die gesakte SchweitzerHort. Gmal. Voluss capiellum. Sp. 100. Marsini 947948.6 St.

713: V. Iurbiscellus. Der kurze Mosgepftera. Gme!. Voutua Eurlyneilus. 1p. 99. Mast. 3. 8. 99. 6. 944. Knors 2. 1. 2. 6. 3. $14 S_{8}$ 


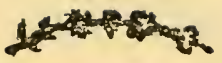

7J4/4 V. Ceramicum. Der geftreckre Morgen. ftern. Gmel. Voluza ceremica 亿p. 103. Martini 3. t. 99. f. 943. Knorr S. t. 4.

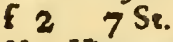

7IS $5 \mathrm{~V}$. Hippocaftane. Die wilde $\mathrm{C}_{2}$ Ravie. Gmel. Murex bippocattanum. rp. 48 Martini 3. t. 99. f. $945.946 .58 t$.

$7166 \mathrm{~V}$. Caffaneum. Die Cafrasie. Gmel. Murez hippocafranum. \{́p. 48. $\beta$. Martiaj 956. 958.4 St.

\section{Volema. Die Pfundbirne.}

1. Pyriformes. Birnförmige.

717 I V Currangniona. Die Cureajjoa - Birne. Grnel. Volura pyrum. Ip 102. 2 St.

7182 V. Myriftica. Die weille Muscatnurs. Greel. Murex hippocaftanum. $f_{p}$. 48. Matsiai 2. t. 40. f. 400 . 40 r. Knotr 6. t. 24. f. 2 , 2 St.

7193 V. Aromazica. Die graue Museatnufs. Gmel. Murex hippocaftaaum 'p. 48. Martini 2. r. 40. f. 399.4 St.

720 - Knorr 6. Tab. 35. fig. 3. I St.

7214 V. Nux-mosebate. Die Merseatnufs. Gmel. Marex hipposartanum. Ip. 48 Ma:o tini 2. $\mathrm{t}$ 40. f 398 . $6 \mathrm{St}$.

722.5 V. Mioschoselling. Die 'sleine MuscaraursGmel Murex bippocaftacum f́p. 48. 3 S?. 723.6 V. Cotonea Die graue Birne raic braun. Lidiea, Gme!. Murgx Gicus, Spo 66. Mart. 3. t. 94 . f. $91 \mathrm{I} . \quad$ Q St.

7247 V. Pyrum Die glaste Eirne. Exel. Mus. ses: fi us. fp. 66 \%. Martuni 3. t. $94 . f_{\text {. }}$ 215.215 a. Chemn.10.1.263. f, I564. 65. \& Sto 
$725 / 8$ \%. Paradifraca. Der Paradies. Apfel. Gruel. Murex ficus. Sp. 66. Martini 3. l. 94 6. 909.910 .2 Si.

Pomiformes. Aepfelförmige.

726 G. Phicase. Das Mantehetten-Horn. Gmel. Buccinum bezoar. 'p. gi. Martini 3. t. 68. F. 754.755. Argenville \& 15. f. $\mathrm{G}$. $9 \mathrm{St}$.

$72710 \mathrm{~V}$ Conful Der Börgeimeinter Gmel. Mures conful. Ip. 159. Chemn. 10. 6 160. 2 1516. 1517. 7 St.

\section{Lade 34.}

$72811 V$. Alouina. Das Alouin. Horn. Gmel. Murex hipposaftannm. 'p. 48. Maruni 3. t. 101. f. 967.68 . $6 \mathrm{St}$.

$72912 \mathrm{~V}$ Glocialis. Die gefrohrne Birne. Gmel. Mulex hippocaftanum. 'p. 48. Martiog 3. L IOI. f. 967.68 . var. I Se.

Pyramis. Die Piramide.

730 I P. Lucifer Das Kamehl. Horn. Gmet. Strombus lucifer Sp. 19. Martiai. 3.6 90. $\mathrm{f} 881.13 \mathrm{St}$.

73. $\quad$ a. 5 St.

732 2 P. Gallice. Die frabzöfilche Piramide d'Argenv 33 fig. 10.6 St.

73312 "P Harpa Die Halfen-Piramide. ISt. $734 / 3$ P. Striase Die geftreifte Piramide Gmel.

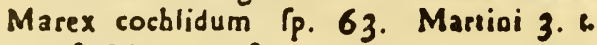
90 f 882.5 St.

7354 P. Volusara. Die eingerollee Piramide Koorr 5 t. 9 fig. $5.6 \mathrm{St}$.

$736 / 5$ P. Conoidea Die kegelförmige Piramide. Martiai 3.t. 91. f. 893. 4 St, - 


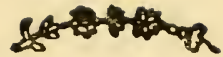

7376 P. Crenulata. Die gerille -Piramide. Martiai 3. 6. 90. f. 883 . 3 St.

Plejona. Die Mufik.Schnecke.

1. Columella multiplicera.

738 I P. Fofflis. Die verkalkte Mufik-Sclinecke. d'Argenv. 33. Gg. 10. I St.

7392 P. Ebrea. Die walde Muák-Schnecke. Gmel. Voluta hebraza. (p. 98. Martini 3. t. 96 f. 924.925 . 5 St.

7403 P. Lineata. Die 4 liniirte Mufik. Schnecke. Gmel. Voluta mufica PP. 96. Martiai 3. t. 96. f. 928.29 .8 St.

\section{Lade 35.}

741/4 P. Muffer. Die 5-linigre Mufik-Sebr. Gmel. Voluta mufica. Ip. $96.12 \mathrm{St}$.

7425 P. Clorse. Die Aarke Mubk Schnecke. Gmel. Volura mafica. Sp 96. $4 \mathrm{St}$.

7436 P. Incarneta. Die Aeichfarbene Mufik.

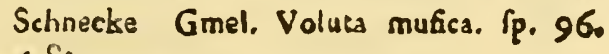
4 St.

7447 P Maculata. Die grobe Mufik Schnecke. Gmel. Voluta mufica ' $P$. 96 . 5 St.

7458 P Levigata Die glare Musik Schnecke. Gmel. Voluta mufica. Sp. $96 .{ }_{3}$ St.

7469 P. Reticulara. Die Neiz-Mufik.Schn. Gmel. Vol. mufica. 'p. 96 . 5 St.

747 1O P. Rofea. Die rothe weifsgelprenkelee Mufik-Scbnecke. Gmel. Vol. mufica. Ip. 96. I St.

748 is P. Confufa. Die fallehe Mufik.Schnecke. Gmel V. mufics. 'f. $96.3 \mathrm{St}$.

74912 P. Turbasa Die verwisshce Mufik. Schnecke. Gmel. Vol. mufica. íp. 96. I Duschifchnittene. 4 St. 
$750 / 13$ ! P. Mixza. Die giūne Mulak.SchneckeGmel. Vol. mufica. Sp. 96. Mart. 3. r. 97. f. 932933 . $4 \mathrm{Se}$.

Vorfteliende find alle von vorzüg.

751 is IP. Aurifrece. Gröfe und Schönheit.

Voluta vexillum (p. 104. Marrin 120. f. 1098. Cbemn. Io. vign. 20. \% B. Koort 5 t. I. f t. ISt.

75215 IP. Lapporica. Das Lapborn Gmel. Vol. lapponica. Sp. 103. Mart. 3. t. 95. f. 920921 . Chemn. 3. 4. 89 . F 872 . 873 Kinots 6. t.-11 א. 2. I St.

2. Calumella quedruplioesa.

16 !! P. Perfica. Das perfifehe Warzen. Horu. oder Bartard Gonäel. Gmel. Vol. feapha. Marrini 3. t. 72 f. 774-776. \& St.

75417 P. Effodja Die Fledermaus von Cout. tagnion Grmel. Vol. vefpertilio. \{p.97. d'Argeuv. 33. fig. 10. I St.

75518 P. Vefpertilio Die Fledermaus. Gmel. Vol. vefperrilio. Mare 3. t. 98. 6. 937. $8 \mathrm{Se}$.

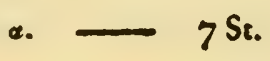

Lade 36.

75719 P. Vampyrus. Der Vampir Gmel. Vol. vefpernlıo. fp.79. Martuni 1397.1398. $6 \mathrm{St}$

75820 P. Cylindracea. Die geftreckee Fledermaus. Gmel. Vol. velpertilio. Ip. 97. I St.

759 P. Murina. Die bunte Fledermaus. Gmel Vol. vefpertilio. Ip. 97. Marc.940. 1.4 St.

- -7 St.

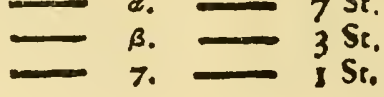




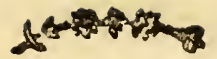

$763 \mid 22$ P. Suilla. Der Schweiosrüfel. Gme!. Vol. velpettilso. Ip 97.13 St.

$764-2.0$ Martiai 3. 1. 98. f. 938.939 .2 St.

22 - P Aracbnoidea. Der Schäekigre Schweinskopf. Gmel. Vol. vefperzilio. Ip $972 \mathrm{St}$

76623 ! P. Bergamotta. Die Bergarnot-Birn. Gmel. Vol Ravicans. Ip. 105 Martini 3. C 95 f. 922.923. I St.

76724 P. Variegeta. Der buntchäekigte Schweinskopf. Die gearackre Bergamot. Bira, Gmel. Vol, verpertillo. Sp. 97. I St.

Lambis. Die Flügel.Schnecke.

I. Alis-retrecsis. Mit ein-

gezogenem Flligel.

768 I L. Pugilis. Der Fechter. Fleich.Scbn. Gmel. Strombus pugilis. Ip 13. Martini 3. t. 84. f. 838. 39. 4 St.

Lade $37 \& 38$.

$770-$ B. -2 St.

$771-\gamma$.

7722 L. Venufta. Die gebandete Flügel.Schn. Gmel. Sriombus pugilis. Tp. 13. I St.

7733 L. Elegantifima. Die felar fchóne FlüSclioecke Ginel. Scrombus falciatus. Ip 9. Martint 3.t 78. \& 800-802. Chcms. 10. \&. 155. f. $1483 \cdot 14847$ St.

7744 L. Lubuana. Die l.öhonifche Flügel. Schnecke. Gmel. Serombus lubuanus (p. 16. Martini 3 t. 77 . f 789790 . II St. 6. 791.7 S. 


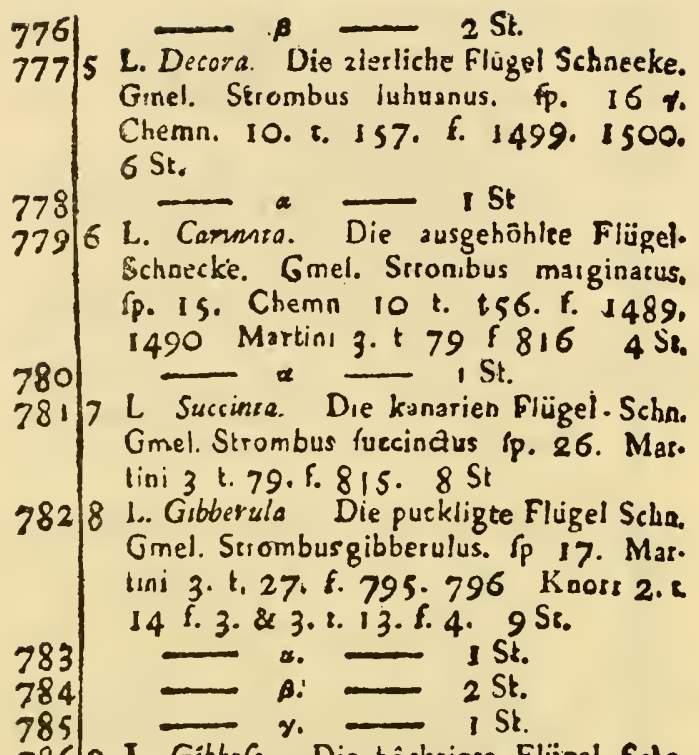
Ginel. Strombus luhusnus. fp. $16 \%$ Chemn. 10. t. 157. 6. 1499. 1500. $6 \mathrm{St}$.

7796 L. Carnnta. Die ausgehōhlee Flügel. Schoecke. Gmel. Srronibus marginatus, fp. Is. Chemn 10 t. t56. f. 1489 , 1490 Martins $3 . t 79 \& 816 \quad 4$ St.

$780 \div-1$ St.

7817 L Succinze. Die kanarien Flügel.Schn. Gmel. Strombus fuecindus 'p. 26. Mas. lini 3 t. 79. f. $815.8 \mathrm{St}$

7828 1. Gibberula Die puekligte Flugel Scha. Gmel. Strombusgibberulus. Ip 17. Mar. tuni 3. t. 27. f. 795.796 Kaost 2.2 14 f. 3. \& 3.8. 13. f. 4.9 St.

$783 \quad 2 \quad 1$ St.

$784-$ a. -2 St.

$785 \quad-\quad \% \quad$ I St.

786 9 L. Gibbofa Die hôckrigre Elügel-Scha Gmel. Strombus gibberulus. fp. 17. Martini 3. t. 27. א. 794 . II Se.

10 L. Albida, Die weifle Fiügel.Schnecke. $6 \mathrm{St}$.

790 I I. Denraza. Die gezähnelse FiŭgelScinecke. Gmel. Stromb ciavus. If. 7. Chemr. 10. 8. 157. 6. 1501. 1502. 2 Se.

$791-2$ St.

$79212 \mathrm{~L}$ Fragilis Die zerbiechliche Fligel. Schaecke. Ginel Srrombus tridentarus. tp 30. Chems. 10 \& 157. f. 1503. 3 St.

79313 L. Pigla Die gemahlce Flügel. Scha. Gmel Sirouib Iatiflimus. Ip. 25 . Martiai 3 t. 89 f. 874. 2 Si. 


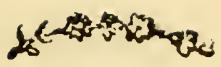

$7938 / 13 \propto$ L. Ventricofa. Die bauchigte Eiùgel-Scbrecke St

794 14 L. Rofec. Die rofenroche Flügei. Scbnerke. I St.

79615 L. Lenziginofa. Die Sommerprofre. Ginel Sirombus leniginolus, fp. 8 . Martıni 3. r. 91. . 892.3 Sr.

797 16 L. Rubiginofa. Die coltfarbene Flii. gel. Schnecke. 2 St.

79817 L Coriacea Die leberfarbene Flügel. Schnecks. ISc.

79918 ! L Puellaris. Die jugeadliche Flü. gel Schnecke. I St.

80019 L. Grisea. Die graue Fiugel Sehnecke. 2 St.

80120 : L. Cingulasa. Die gegürtelte Flügel. Senneckè. 2 St.

80221 L Rana. Der Frofel Gmel. ftromb. lentiginafus. íp 8. Matuni 3.8.80. 81 f. 8278287 St.

$803-2$ a. $2 \mathrm{St}$.

80422 L. Pipa. Die Krōte. Gmel. Aromb. lentigınofus 'p. 8. Martin 3. ז. 80. f. 825.826 .2 个t.

805

80623 L Labiata. Die Dicklippe. Gmel. ftromb urceus 'p. 29 Marcini 3 t. 78 f $804.805 .85 \mathrm{~s}$.

80724 L. Urceus. Der Scbwarzmund. Gmel. frombas urceus. if. 29. Mattioi 3. e. 78 f. $803-6$. Knorr 3. t. I3. f. 5. $78 \mathrm{t}$.

80825 L. Carnea. Die hornigre Ftügel. Selinecke. Gmel, itromb. faiciatus, 1 p. 9 . Martiai 3. t. 82. 6. $833 \cdot 834$. 8St. 
80926 L. Carnaris. Die Fleifchratbene Fiü. gel Schnecke. Gmel. Stromb. fafsiatus. 3. 9. Mastui 3. t. 91. f. 893 . 3 St.

2. Alis expenfis. Mit ausgedehasen Flügel.

81027 L. Turrits. Die getbürmte FlügelScbuecke. Martini 3. ' 84. f. 841 . 42. I St.

L. Curruca. Die Mücke Gmel. Stromb. gallus. Yp. 11. Martini 3 t. 83. f. 836 . 37. Favaoue Tb. 21 . Gg. A. 4.4 Sto

813 a. $6 \mathrm{St}$.

81429 L. Bulla Der Bampßabar. Gmel. Stromb. auris Dianx. [P. 12. Mastooi 3. t. 84. f. 840.7 St.

81530 L. Stive. Die Plugfterze. Gmel. Stromb. auris Dizox. Kp. 12. $2 \mathrm{St}$

81631 L. Auris Diann. Der Dianen. Flugel, Gmel. Srromb. auris Dianz. f́. 12. Mut. tuin 3. t. 84. f. 838. 839. 3 St.

$817 !$ a rehr felrea. I5t.

818 - 3 - 5 St.

81932 L. Buris. Das Efelsobr. Gmel. Stromb. auris Dianz. TP. 12.3 St.

820133 L. Arasrum. Der Plug. Gmel. Stromb. suris Dizaz. Martini 1487. 1488. 1 St.

82134 L. Vomer Das PRugfebareifen. Gmel. Stromb. auris Dians. Martini 1485. 1486. I Sto

822 35 L. Gallus. Des Engelsä̈igel. Gmel. Stromb. gallus. Ip. II. Martini 3.8.84. f. 841. 842. Kaors 4. B. 12. E. J. $5 \mathrm{Sin}$ 


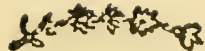

$823 / 36$ L. Velun. Das Segel. Gmel. Atrombas. gallus. Ip. II. Martini 3.t. 85. \{. 846 847.3 St.

\section{Lade 40.}

82537 L. Velamen. Dic eingerollte bunee Flï. gel-Schnecke. Gnel. ftrombus gallus. ip. II. 2 Se

$826 / 38$ L Lobazs. Die zerrifene Flügel. Schr. Gmel. Atromb gallus Sp. I I. Chemn. IO. 158 f. $1506.15072 \mathrm{Se}$.

827,39 L. Latifrina. Die grofte Flügel-Scinne. cke, imit breitumlchlagener Lippe. Gmel. Stromb. lacilîtnus. ip. 21. Mart. 3. e. $82 . \mathrm{f} 832.833 . \quad 1 \mathrm{St}$.

82840 L. Canariuın. Das Tãubchen. Gmel。 Stromb canarium. 'p. 24. Mart. 3. c. 79. $1817 \cdot 18$. Knorr 1. 6. 18: f. $5.38 \mathrm{c}$

83041 L. Canarienfis, Des Canatienrogel. I SP.

83142 L. Canaria. Der weifle Cabarienvogel. I St.

832 - a. Tehr montrtôfe. Is.

833/43 L. Turturella Das Turceltaubchen. Gmel. Stromb. cadarium. Sp. 24. Mari tioi 3.t. 79. f. 817.2 St.

83444 L. Epidromis. Das Befamsfegel. Ginel. Stromb. epidromis, f́p. 22. Martini 3. r. 79. E. 82 I. 7 St.

83545 L. Plicasa Det Wetterhaho. Gmel. Stromb. vittatus. If. $_{\text {. 25. }}$. Chem. 10. t. $157 \mathrm{f} 1496.2 \mathrm{St}$.

836 46 L. Minimus. Die kleine Flügel-Schnecke, Gmel, Stromb, minimus. fp. $_{2} 3$ E

Cbemn, 


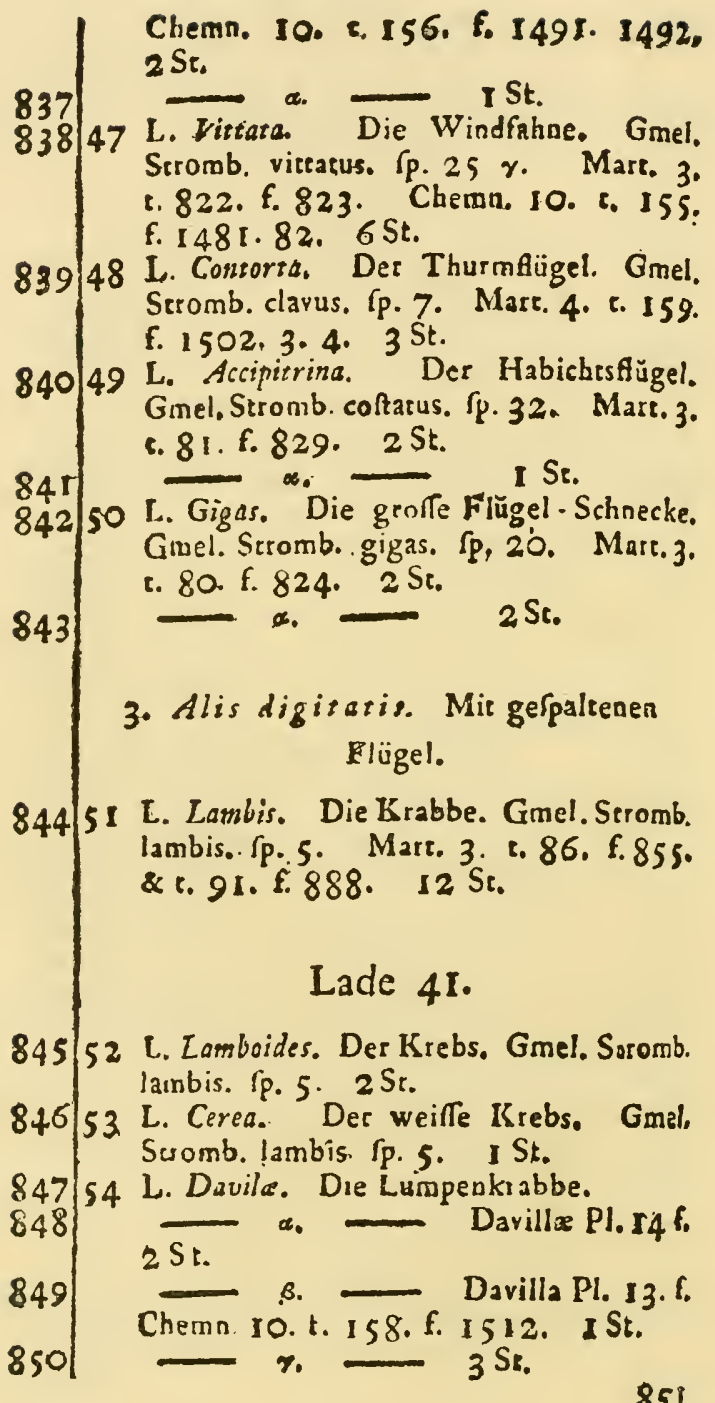



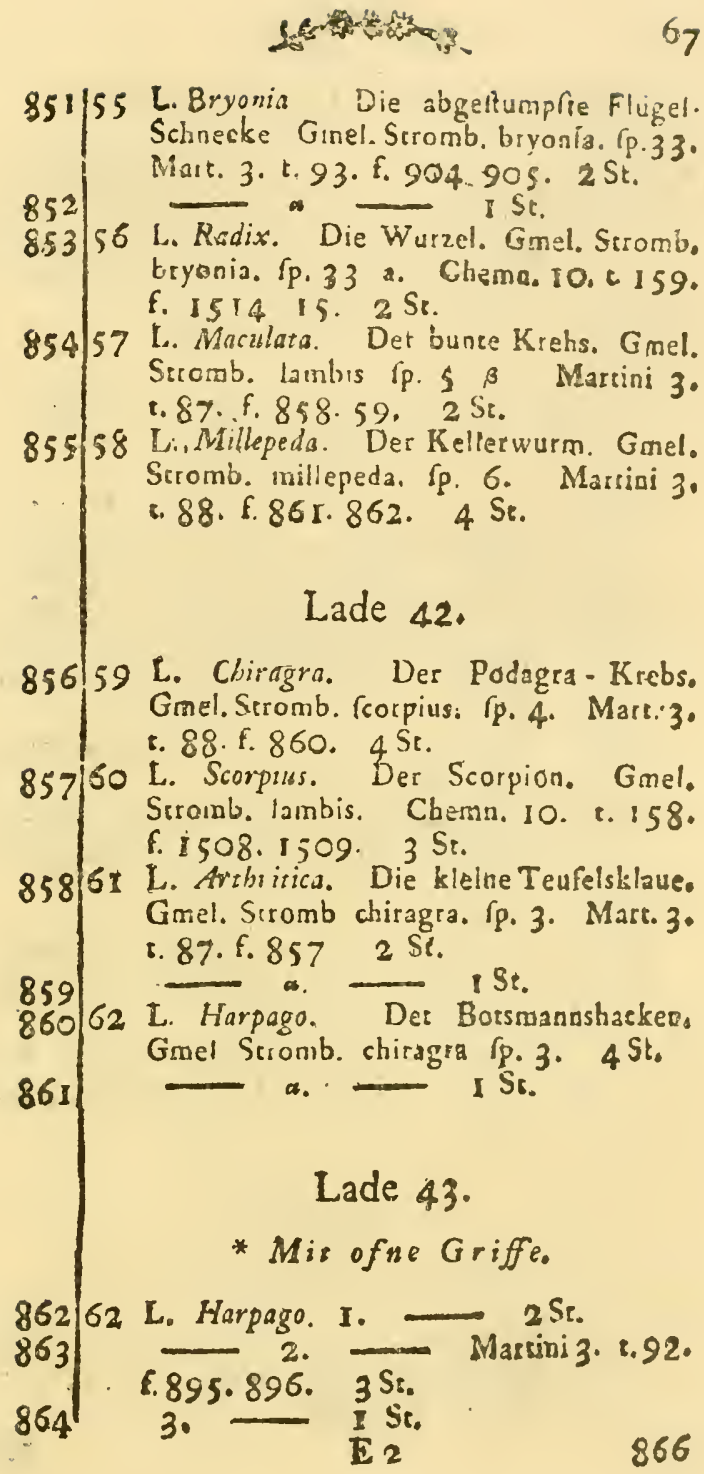
$866 ; 62$ I. Harpago. $4 .-{ }_{3} \mathrm{St}$ ISt.

86963 L. Pes.Prlicani. Dis Fünffiager.Schae. cke. Gmel. Stromb. pes peticam. If. 2. Mart. 3. t. 85. f. 848. -850 . $10 \mathrm{St}_{\text {t }}$

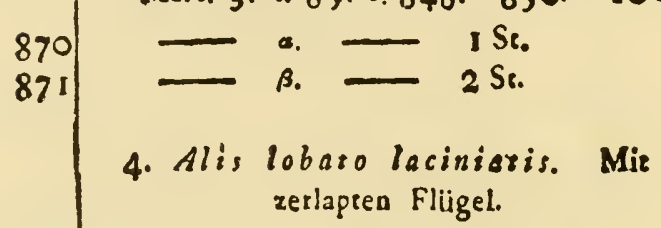

87264 L. Lobara. Die Flügel-Schnecke mis gefaltener Lippe. Ginel. Stronb. lainbss. IP 5 b. Mart. 3. t. 92. 6. 902 I Sz.

87365 L. Undulata. Die Flügel-Schnecke. nit wellenfôtmiger Lippe. Gmel. Stromb. lambis. I. . S. Mart. 3. t. 92. f. 898 .

$874 \quad 2$ L. Exemplare. $5 \mathrm{St}$.

87566 L Hermapbrodita. Eie Zwitter FlügetSchnecke. Gmel, Stromb, lambis. 1p. 5 1 St.

876 - 2.

87767 L. Laciniata. Die Flügel - Schnerke, mit gerunzelter Lippe. Gmel. Stromb. lambs. Ś. 5. a. I St. uncomplere Ex. 7 S.

\section{Lade 44.}

Dentalium. Der Zahn.

8791 D. Aprinums. Der Widderzatin. (NB. Mainoi Conch, lab. 1. f 4. B. ütert. Lin. vzi (p. 2.) 4.5 . 


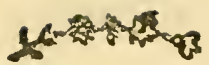

880 '2 D. Eburiseun. Det elfenbeinerne $Z_{\text {ahn. }}$ I St.

8813 D. Elepbantinum. Eer Elephanten.Zahn. Gmel. Dental. elephantinum. 1p. I. Mar. sini 1.t. 1. f. 5, 2. $3 \mathrm{St}$

8824 D Lupinum. Der Wolís-Zahn. Gmel. Dentsl. Ariaculum. Sp. I3. Mats. I. t. I. f. 5 ist.

8835 D. Extalis. Der kleine Zabn. Gmel. Deptal. Eafciatum. Ip. 10. Mast. 1. t. I. f. 3. b. $2 \mathrm{St}$.

Verpa. Der Venus-Schaft,

884 I V. Penis. Der Venus.Schafe. Gmel, Serpula penis. Ip. 17 Martiui I.t. 1. f. 7. Knotr 4. 2. 28 . f. 1. \& 6. 40. f. 1. 4 St.

Sespula. Die Wurm-Röhre.

885 I S. Intefinalis. Der dicke Darm Gmel. Serpula anguina. Ip. 15: $\beta$. Marriui J. t. I. f. I I. I Se.

8862 S Ileacea. Der dünoe Darm. I Sr

8873 S. Clava Herculis. Die Hercules KeuleNaturforfcber I3 St. tab. 1. fig. 10. I St. 88814 S Diodenolis Der 12 Finger - Darm. d'Argenr. Pl 29 fig. E 5 St

89915 S. Poiita. Der glatte Darm. Martini 3. 8. 3. Ix. 21 . ist.

89066 S. Lamellaza. Die blâttrige Wurm.Rühre. Gmel. Serpula contotruplicats. 1p. 10. Martini 3. fig. 24. A. $6 \mathrm{St}$.

8918 S Anguina. Die gefthlangelte Wurmröhre. Mart. 3. fg. 13. A. 3 St.

8927 S. Porofa. Der rothe Darm, Martini 2. fig. 13. C. ISt, 
893/9! S. Lumbricalis. Der Zeprer Jovis. d'At. genv. tab. 29. fig. J. I St.

89410 S. Colsibrina. Nie fcliwarze Wurn Röbre. Knorr tab. 22. fig. 1. IS.

895 IIS Contortuplicasa. Die pewundege Wurm Rolure Gmel Serpula consortupli. cata. ff. 10. Martini I. t.3. f. 24. A. $7 \mathrm{St}$.

89612 S. Glomeraza. Die gewickelse Wurm. Rôbre. M.ıruni 1. ז. 19. f. B. 5 St.

897 is S Corallordes. Die kosallenförmige Wurm Röhre. I Se.

898 I4 S. Merdarsa. Der Hünerdarm. 2 Sr.

Lade $45 \& 46$. b.

Nautilus. Die Naurel.

899 I N. Pompilius Die groffe Naurel Gmel. Nauril. pompilius p. I. Martioi 1. s. 18. f. $164.9 \mathrm{Sr}$.

f. 166.7 .

- Martioi 1. t. 18.

901

903 mologicch. ist. thologircb. "2. St. Blumen. I St. 9042 N. Spirule. $13 \mathrm{St}$.

\section{Lade 47.}

Argonauta. Der Segler.

905 I A. Argo. Die fchmalkieligre Papier-Nabtel. Gmel. Argonauta argo. Jp. Io \% Martini I. 8. 17. 6, 157. 2 St, 


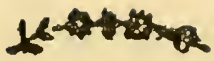

906/2 A. Tuberculara. Der Reisbrey-Nastel. Gmel. Argon, argo, rp. 1.B. Marcini I. 1. 17. .. Is6. 2 St.

9073 A. Papyracea. Der Papier-Nàurel. Gmel, Argon. argo. f́. I. $2 \mathrm{Se}$.

9084 A. Brbula Der Laefchpapier - Naurel. Guiel. Argon.aigo. 'p. 1.6. Mart. 1. t. 18. f. 160 . 3 St.

Angaria. Das Polthorn.

I. Columella depreffa, mit gedruckter

Windung.

9091 A. Squamate. Der ichuppigte Delpbia. Gmel. Tusbo delphinus 5.44 . Mar. tini 1730. 1731 Geve t. 4. f. 24 A. $3 \mathrm{Si}$

9rol2 A. Diftorra. Der rothe Delphin. Gmel. Turbo diftortus. f́. 46. Mass. 5. 1175. f $1737-1739.2$ St.

9 II 3 A. Delphinus. Der gezackte Delphın. Gniel. Turbo delphinus, fp. 44. Matr.5. t. 175. f. $17.84 .1735 .15 \mathrm{St}$.

9124 A. Rofea. Der rofenfarbene Delpbia. Gmel. Turbo delphinus. 'p. 44. Mart 5. t. 175. f. 1733 3t

3136 A. Arion. Ver Arion. Gmel. Turbo delphinus. Ip 44.\%. Mart. 5.t.175. f. 1736 . St.

9145 A.Cinta. Der gebandere Delphia, Gmel. Turbo delphigus. Ip. 44. Marr. 1732.

9157 ! A. Pentagone. Det fiiafeckigre Delphin. I St,

2. Columelle elevasa, mit exbabener

Windung.

916/8 A. Lineata, Vas !iniisze Pofthorn. Gmel. Helix ericeroums. fo. 65, Gualiv. Tb. 3. 

| fig. O Chemr. 9.t. 132. . I 193-I 195.
9179 A. Fasciata. Das gebandete Pnthorn. Gmel. Helix volvulus. Ip. 9r. Seba Tom. III. Tab. XL. fig. 18. Clema 9. t. I23. f. $1064-66$. I St.
$91810 \mathrm{~A}$. Pnlita Das gefaltene Pofthorn. Gonel. Helix lapicida fp. 2. Seba Tom. III. Tab. XL. fig 33.34. Chema. 9. c. I26.f. 1107 . I St.
919 II A. Bifascrata Das $2 \mathrm{mal}$ gebandete Pofthorn. Chemn. 9. t. 123. f. 1071. 72. I St.

Planorbis. Die Scheibe.

920 I P. Verus. Die kleine Scheibe. Geves. 4 f. 21 a. b. 22, 23.4 St.

$9212 \mathrm{P}$ Cornu Arietis. Das Widderhoro. Gmel. Helix cornu ariesis, Sp. 41. Lifter 136. fig. 40. 3 St.

9223 P. Corners. Die Horn-Scheibe Gmel. Helix cornea. \{p. 35. Chemis. 9. 1. 127. f III3-1123. $9 S_{t}$.

$923 / 4 \mathrm{P}$ Serpentinus. Die gefchlanngelte Scbeibe. 9244 "P Lacteus. Die weille Scherbe.

925 S P. Ungriturus. Die dunkelbraune Scheibe. Gmel. Helix ungulina. 'p. 75. Chemn. 9. c. 125 . f. 1098.99. Gualtiv 3. fig. 222. 2 St.

9266 P. Cirvinus. Die, citronengelbe Scheibe. I St.

$9277 \mathrm{P}$. Zunarius. Die umgürtete Seheibe. Gmel Helix zunaria. Ip. 63. Gualeiv 3. fig L L. Knorr 5 t. 21 f. 3.4 .6 Sto

9288 ! P Peilucidus. Die durcblichtige Scherbe mit einem monftiofen Eingang. I St,

9299 P. Bilinearus. Die zureybandigte Scheibe Simel. Helix zonaria. 'p. 63. I St 


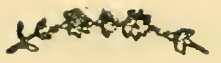

930 ro P. Pelhs - anguinea. Die Schlangen Haut. Gmel. Helix pellis ferpentus. Ip. 254. Chemn 9 t. 125. f. 1095. 1096 3 St.

931 11 P. Lapisida Die Aeinartige Scbeithe aus Rupill. Gmel. Helax planorbis ip. 20. Chemn 9. t. 126. f. 1102.3. 4 St.

$93212 \mathrm{P}$. Famaicenfrs. Die Scheibe aus Ja. malca. Ginel. Helix pamaicenfis $r_{p} 234$ Chemin. 9. t. 129 E. I 1 40.45 . Seba $\mathrm{XL}$ fig. 3:4.5. $3 \mathrm{St}$.

93313 P. Boltenionus. Die Bolsenficle Scheibe. Martini 92 r. 922.

93414 P. Effiffus. Die vielbandigre Scheibe. Gmel. Helix ampullacea. fp. 43 r. Chemn. 9. t. 129. f. 1144.1145. 8 St.

935 is P Cunctus Die umwandene Scheibe. Gmel. Helix cincta. 1p. 56.2 St.

\section{Lade 48.}

Pnmatia. Die Garien-Schnecke.

936 I P. Chrisata. Die gelbhraune Garen. Schnecke. Gmel. Helix cornu militare. Sp. 29. I St.

f. 1142.43. I St Chemu. 9 t. 129.

2 P. Niven Die rchneeweife Gareen. Schnecke Gmel. Helix nivea. Ip. 176. I St.

9383 P. Laćtea. Die milchweiffe-GartenSclusecke. 2 St.

$8394 \mathrm{P}$ Finnta. Die fadigte Garten Scbnecke. I St.

940 a. 2 St. 9415 P. Exorica. Die auslândilche Garrce 
942/6 P. Aramaria. Die befraubre Garter. Schnecke. Gnicl. Helix ladtea, fp. 237. Cliemn 9.t. 130. f. II61. 5 St.

9437 P. Deprelfa. Die platte Garren-Schnecke. 9448 P Frondofa. Die Büadclartige Gatten. Schnecke. I St.

9459 P. Ufra. Die gelbliche Garten-Schnecke. I St.

946 10 P. Horten/15. Die lichigelbe GattenSchnecke. Gmel. Helix bortenfis. Ip. 109. I St.

947 II P. Arverris Die Feld.Schnecke. Gmel. Helix hortenfis $\gamma_{p}$. 109. I Sr.

948 12 P. Nemorofa. Die röthliche GartenSclinecke Gmel. Helix bortearis. Ip. 109. I St.

949 I 3 P Silveftris. Die braungebandete Gat. ren-Schnecke. Gmel. Helix horrenfis. rp 109 I St.

950 14 P. Rbodia. Die rolentorhe Garten. Schnecke. Gmel, Helix rhodia. 'p. 239. Chemn. 9.t. 132. f. II 179 80. I St.

$95115 \mathrm{P}$ Balibeus. Die runzliche Garten. Sclinecke. Gmel. Helix algira. 'P. II. Chemn. 9. t. 125. f. 109394.2 St.

$95216 \mathrm{P}$ Cicatrzofa. Die narbigte Garten. Schnecke. Gmel Helix cicatricofa. Sp. 4. Clemn. 9. t. 109. f. 923.2 St.

95317 P. Succinea. Die bernfteinfarbne Gap. ten Schnecke. Gmel Helix citrina. Ip 49. Chemn. 9. 1. 131. f. 1169. I St.

95418 P. Rutpola. Die braunrothe Garten: Schnecxe. Gmel. Helix citrina, fp. 49. Chemn, 9. 2. 131. f. 1173 . I Si.

255 19 P. Dimidiasa. Die zwevfatbigte Garten-Schnecke. Gmel Helix citcina. fp. 49. Chemn. 9. t. I3 I. f. 1170.2 St.

956 20 P. Rutila. Die rothbraune GatrenSchnecke. Gmel, Helix citrina. Sp. 49. Chemn, 9. t. I3I. f. II 74. I St. 
957jar P. Fiaveola. Die blasgelbe Garren. Schnecke. Gmel. Helix cicrina. (P. 49. Geve t. 26. f. 278.2 St.

95822 P. Cisrina. Die citronengelbe GarrenSchnecke. Gmel. Helix cirriıa. íp. 49. v. Born Tb, 15. fig 3. 4. Chema. 9. c. $138.1 .1170 .2 \mathrm{Se}$

$95923 \mathrm{P}$ Paleacea. Die gelbe gebandere Car. ren.Schnecise. Gmel Helix cittina. IP: 49. Chemo. 9. t. 131.f 1170.2 St.

96024 P. Straminea. Die frobfarbene GartenSchnecke. Gmel. Helix citrina. Íp. 49. Geve t. 26. א. 280.4 St.

96125 ! P. Imbricata. Die gereifte GartenSchoecke. Geve't. 24. f. 243. I St.

96216 !P. Tranfparens. Die dutchǴchuge Garter. Sclinecke. I St.

Fantinina. Die Wald-Schnecke.

I. Elevara, mit erbabenea Zopr. 963 I J Violacea. Die blaue Wald-Schoecke Ip. 103. Knorr 2. Tb. 30. fig 23. $4 \mathrm{St}$

9642 ! J. Singularis. Die befonders feliöne Wald-Schnecke, fehr Selren. I St.

9653 J Turbinoidea. Die knopfarrige Wald. Sclinecke. I St.

966 4 J. Limbara. Die gefâume Wald Schnecke. ise.

9675 J. Cytberea. Die zarte Wald. Schnecke. Chemn. 9. t. 123. f. 1063. I5 St. 9866 J. Pellucida. Die durcífichrige Wald- 
2. Depreffiuscule, mir gedtacktea

$$
\text { Zopf. }
$$

9877 J. Una Der Afchenropf I St.

$9888 \mathrm{~J}$. Bombards. Die Kugel-Schnerke. ist.

9899 J. Siloperaria, Die kleine Kugel. 2 S6 9 * - I St.

29010 J. Pcrrarna. Die gebandere Wald. Schnerke. Gmel. Helis pomatia. Sp. 47. Chemn. 9. t. 128. E. 1137.2 St.

Chemn 9. t. 108. f. 908. 9. 10. 2 Sc.

992 - B. - varietas elon. gara. Gmel. Helix fcalaris. fp. I16. Cbemn. 9. t. 128. f. 1139. No. I. I St.

993 II J Flammeola, Die gefammte Wals. Schnecke. Gmel Helix ligata. ip 57. Chemo. 9. ז. 128 . f. 1137. II Sr.

99412 !! J. Fomaicenfis. Die Jamaica - Schn. Ginel. Helix pamaicenfis. Ip. 234. Chemu. 9. ז. 129. f. 1140.1141. I $8 \mathrm{c}$.

995 3. - a. I St.

996 1 3 J Linearia. Die liniixte Wald.Schn, 2 St.

$997: 4$ J Pitta. Die gezeichnete Wald - Scha. Gmel. Helix picta. Jp. 189 . ISt.

999 is J. Cortex ciwi. Die Cirronenfchale. Gmel. Helix prôa. fp. 189. Chemn, 9. k. 130. f. 1162. 1163. I St.

Lade. 


\section{0}

Lade 49.

Lampadion. Die Lampe.

r. Fauce fimplici, mir gewöhuliches

Oofnung.

1000 I L. Carocolla. Die braune Lampe. Grsel. Helix carocolla. fp. 26.2 Chemin. 9. i. 125. f. $109 \mathrm{I} .6 \mathrm{Sc}$.

1001

2 L. Tefsuceum. Die Nachtlampe. Garel. Helix carocolla. fp. 25. Chemn. 9. i. 125 , f. 1092. 3 St.

10023 L. Marginatum. Die gebandere Launpe. Gmel. Helix marginella. fp. 162. v. Born. t. I 4. fig 7. 8. 2 St.

10034 L. Orbiculare. Die runde Lampe. Gmel. Helix marginella, $\Upsilon_{2} .162$. Chemn, 9. 8. 125. f. 1097. ISt-

10044 * L. 3 Lineatum. Die gedrehte Lampe. 2 St.

10055 L. Sinicum. Die gekehlce Lampe. I St. $10066 \mathrm{~L}$. Cbinenfe. Die chinefifche Lampe. Gmel. Trochus veftiatius. Tp. 75 in allea 15 Stŭck. Chem. 5. t. 166. \& I60I. a. b. c f. g. h. Lifter t. I49.8C652. f. $44-50$. 3 Se.

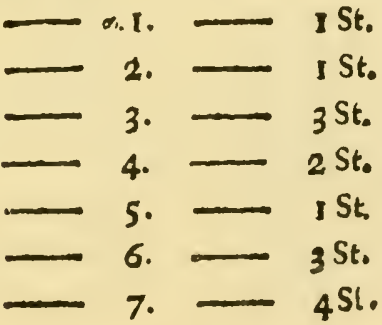




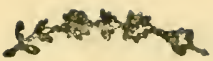

3027/4 A. Radiata. Die neifsfürsigte Perfpectiv. Schnecke. Gmel. T. bybridus. ip. 4. Chemn. 5. t. 173. E, 1704. 1705. Geve t. 25. 8. $274 \cdot$. $2.74 \cdot$ b. 3 S.

\section{Lade so.}

Aftrea. Das Sonnenhorn.

1028 I ! A. Imperialis. Das kayferliche Son. nenborn. Gmel. Trochus iniperialis. 4p 63. Tehe felten. Chera. 5. 2. 173. 174. f. 1714. 1715 . I St

10292 A. Sirises. Der Sirusftern. Gmel. Tio. chos tolaris. IP. 15. var. Tebs leltea. I St.

10303 A. Polaris. Das Sonaenhora des Rumpfs. Gmel. Troebus folasis. \{p. I5. Chemo.5. 2.173. $1.1700 .1701 .3 \mathrm{Se}$.

10314 A. Colcer. Des Spora. Gmel. Turtw aculearuz. P. 48. Favanoe PI. $12.6 \mathrm{gg}$ E.4. Chema. 5.t. 168. F. $\$ 554.55$. 4 Sto

1033 .5 A. Nicobarica. Der nicubarifebe Spora. Gmel. Turbo aculearus. $\{p \cdot 48$. Chemo 5 . t. 164. 6. 1554.1555 .4 St.

$3034 / 5$ A. Micars. Das grüre Sounealuora. I Se.

I0356 A. Venus. Die untergeiende Sonne. 3 St.

10367 A. Plocbia. Die aufgebeade Sunne. Gmel. Troshus folaris. To. 15. $\beta$. Cbemn. 5.t. 174. f. $1716.87 .8 \mathrm{St}$.

1037/8 A. Corrusca. Das giäazende Sonnzahorn. Goel. Trochus in:erais. 6. 62. Chemn. s. t. 173. 6. 1712.29930 1 St. 
1039/9 A Transa Das Titanshorn. Gmel. Tro. chus tolaris. Sp.65. Chemn. 5. 2. 174. f. $1718.19 .4 \mathrm{St}$

104010 A. Lapidifera. Der Steinträger. Gmel, Trechus conchiliophorus. Ip. 110. Chemn. 5. t. 172. f. 1688. 1789. $1 \mathrm{Sr}$.

1041 is A. Covcbyliopbora. Der Schneckentrât ger. Gmel. Trochus conchyliophorus, f. 1 10. Chemn. 5. 2. 172. f. 1688 90. Favanne PI. 12. fig. C. I, ISt.

104212 A. Corallopbora. Der Corallentrages, Gmel. Trochus conchiliophorus Sp. 110, I St.

\section{Trochus. Der Kräufel.}

1. Unbilico perforata. Mit offerlen Nabel.

1043 I T. Nilozicus. Der Nılkıāufel. Gmel, Trocbus nuloricus. Ip 1. Chemn. s. t. $167 . f_{1605} 9 \mathrm{St}$.

$1044^{2}$ T. Flammeus. Der geflammec K1 āufet, Gnel Trochus inloricus. \{p. 1. Cbem s. 8. 167. f. 1606. 1607. 9 Si.

10453 T. Undatus. Det gewellte Kraufel. Gmel. Trochus nulociciss ip. I. Geve t. 6.844 .244 . b. 6 St.

$10464 \mathrm{~T}$. Afper. Der granulirte Kräufel. Gmel. Trochus virgatus. 1p 83. Chemu.5. 1. 160. f. 1514-1515. 8 St.

10475 T. Grandinatus. Dcr fcharfe Kräufel. Gmel Trochus maculatus. Ip. 2. Chema,5. ?. 168.f. 1616. ISt. 
1049/6 T. Stellaris. Der geftirnte Iräufei. Gmel. I rochus ftellatus. fj. 26. Chemn. 5 169. f. 1630. $2 \mathrm{St}$.

I050 7 T. Gvenularis. Der bunte tauhe Krāurel Gmel. Trochus เadiatus. $\left\{p_{0} 33 \cdot \gamma\right.$. Chemn. 5. 2. 170. \&. 1642. a. B. $1 \mathrm{St}$

IOS 18 T. Vernalis. Der grasgrüne Krâufel. Gmel. Trochns vernus. $\int_{p} .22$. Chemn. 5. 1. 169. 6. 1625.1626. I St.

1052 9 T. Nafurus. Der lpitze Kräufel Gmel. Trochus confperfus. \{p. 2j. Chema. 5. t. 169 f. 1627. 14 Sc.

\section{Lade 50.}

$105310 \mathrm{~T}$. Gurrorus Der rropf. Krâulel. Gmel Tru.hus confperfus. Sp. 23. Geve i. 7 f 50. a. 50 b. 3 Se.

1054 II l' Cagtrenfis. Der geperlee Kräufel. Gmel Trochus ochiroleucus $r_{p} .25$. fehr felten Cbemn, 5. t. 169 f. 1629. i Se

IOS5 12 T. Tentrorialis Der gefaltene Ktāufel. Gmel. Trochus oebroleucus. $\int_{p} .25$. I Si.

I056 13 T. Turboidea Der gefchnürte Kräutel. Gmel. Trochus magus. Sp 7. Tehr felren. Chemn. 5.8. 171. f. 1658. 4 St.

I057 is T. Tectum cbinterfe. Das chinefrche Dach, rehr felceo. I St.

I058 $15 \mathrm{~T}$. Striatus. Der geftreifte Kraufel. Sp. 78 ist.

105916 T. Turritus. Der gethürmte Kràufel. Gmel. Trochus pyramis. Ip. 39. Chemn.

$1060 \mid 17$ T. Verrucofus. Det warzigte Kreufel. 
106I/18 T. Ingersupzriss. Det kleiafte Krärde1 I $\mathrm{St}$.

1062 19 T. Tracblea. Der gedrecifelte Kräufel. cehs felten.

106320 T. Declivis. Der fchiefe Krâufel. Gmel. Trochus agypios. 1p. 4 T. Cbamn. S. . 17 1. f. 1663 I664 ISt.

10642 I T. Lunatus. Der ausgekehlte Krâufel. Gmel. Tıochus jujubiaus. Ip. 19. Chema. 5. t. 167. f. $1612,1613.2 \mathrm{Sr}$.

I. Unbilico claufo. Mit gefchloterem Nabel.

106522 T. Plicatus. Der gefalsere Kräufel. Gmei. Trochus imbricards. Sp. 93. Chemb, 5: tab. 162. I $153 \mathrm{~T} .2 \mathrm{St}$

1066/23 T Rugofus. Det rwozlige Krâufel, Gme!. Trochus imbricanus. Tp. 93. B) Inors Verg. 3.c. 29 f.g. 1. 2 . 31 Bt. $106723 * T$ T. Spinofus. Det dornige Kraufel. fp. 18. 1 . Se.

106824 T. Anilis. Der alte Kräufel. Gmel. Trochus americanus. Sp. 94. Chemn. 5.e. 162. .. 1534.1535 .4 St.

107026 T. Forskoblii. Der forskolifche Kräu. fel. Gmel. Trochus foveolatus. Sp. 84. B. Tehr feltea. Chemn, 5. t. 161. f. $1518 . \& 19.2 \mathrm{St}$.

107127 T. Gozbicus Der Gorhifche Krâufel。 Gmel. Trosbus mausitianus (p. 99. Chenu. 5. t. 163. f. 1547.1548II Se.

1072 a. ISt.

107328 T. Ferieffrazus. Dsr rautea Kräufel. Gme:。 Trochus feneftratus. Tp. 100. Chosia. 5. 6. 163. . . 1589. 1550 . 5 St 


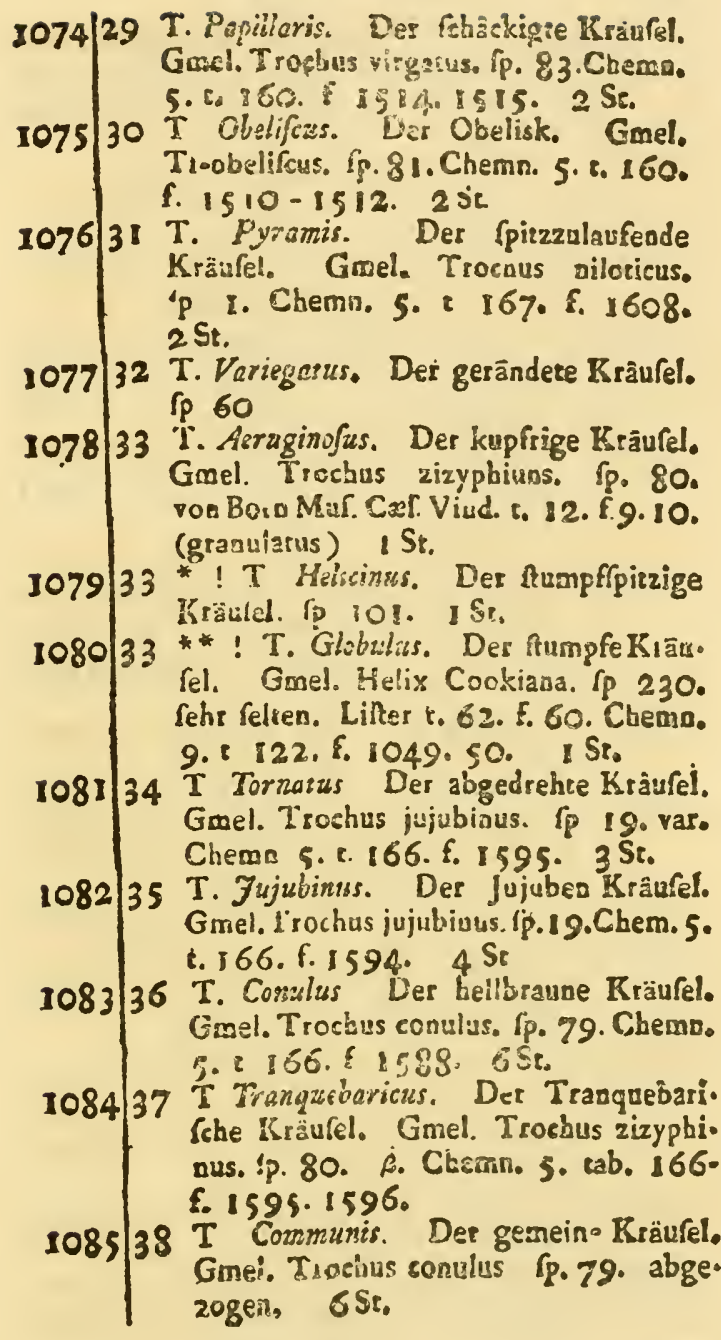


39 T. Novar Zeelandiae. Der seufeelandi. fche Krâfel. Gmel. T. Labio. 'p 76. Matt. 5. 1. 166.1579. I580. Telted.

\section{Cidaris. Der Iurban.}

I. Anfradizilus Spizofss. Mit dornigren Windungeo.

1 C. Cookii. Der Cooklehe Turban, Gmel. Trochus. 5p. 97. Chema. 5. 8. 164. f. 1550 . I551. 2 St.

10882 C. Pagodus. Die.Pagode. Gmel. Turbo pagodus. Sp. 12. Chem. 5. r. 163. 6. I 54 I. I542. 4 Si.

10893 C. Tetrum perficum. Das perfifebe Dach. Gmel. Turbo rectum perficum. 'P. II. Cbemn. 5. 1. 163. f. 1543. I544. 3 St.

1093 4 C. Spimula. Der Aachligere Turban. $3 \mathrm{St}$.

I092 5 C. Arabica. Der Arabifcbe Turban. Gnel. Trocbus xgyptiacus fp. 41. Chems. 5.1. 171 . 1663 1664. 6 St.

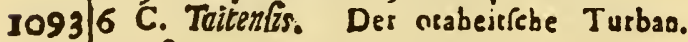
ISt.

10948 C. Rugofa. Der runzligre Turban Gmel. Turbo rugofus. É. 14. Chemn. 5. tab. 381. f. 1783.1783 .4 St.

10957 C. Celaro Der gemelifelie Turban. Gniel. Tiochos cxlarus. Ip. 95. Chemn. 3. 8. 162. f. 1536. 1537. Knort Verg. 5. F. 12. f. $3.8 \mathrm{St}$.

10969 C. Plicara. Der gefaliene Turioan. Gmel. Turbo rugolus. ip. 14. var. $9 \mathrm{~S}$. 10979 * C. Voblii. Der Atachlige Turbar.

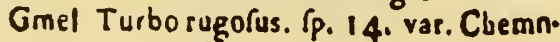
10. $\$ 1165.6 .1585 .86,2 / 5$ s. 

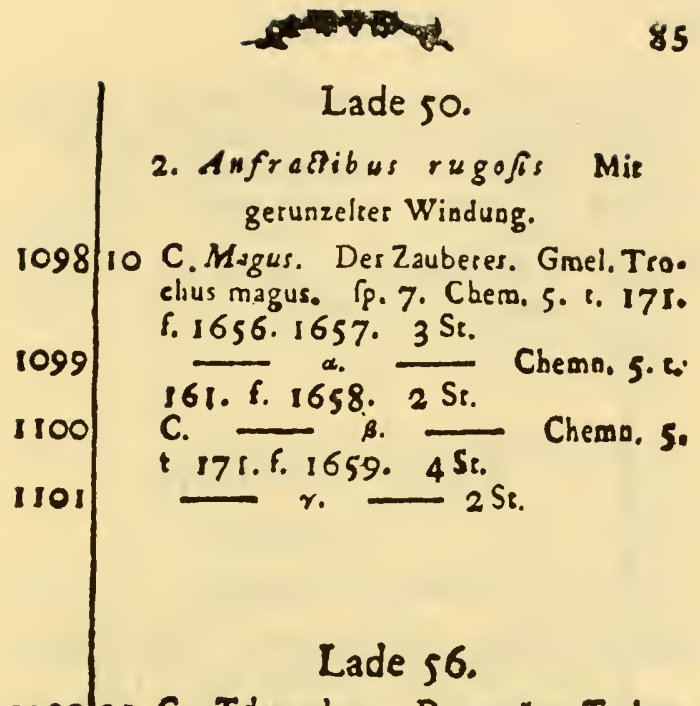

1102 II C. Tiberculase. Der grüne Turban. Gme!. Trochus Tuber. 'p. 77. Koors verg. 1. t. 3 f. 2.8 4. t. 4. f. 3 4. Chemn. 5. 1. 565 . f. $1572.73 .4 \mathrm{Sr}^{\circ}$ - a. olivenfarbigt. 2 St.

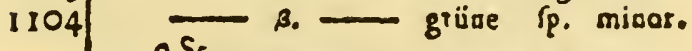
2 Se.

12 C. Nova Zeelendiae. Der aeufceländifche Turban. Gmel. Trochus Cookii. 5. 97. Der eine ilt ganz abgezogen. und der andere beynalic, Sebr felcen Cbemn. 5. t. 164. f. 1550. 2 St.

13 C. Flaminea Der gelammic Turbas. Gmel. Trochus magus. Sp. 7. Chemn.5. 171 f. $1659.2 \mathrm{St}$.

110814 C Punctulara. Der gentppelee Turban. Ginel. Trochus reñellatus. Sp. 52. Cbemso. 5. 8. J7 I. f. 1683.3 St. 110914 *C. Lineare. Der gelleckse Tarbsa. eod. I St. 

IIII I5 C. Granulata. Prinz Rudolph. 12. fig. 1. Gmel. Trochus alper. Íp. IOS. Chemn. 5. t. 166. f. I582. 6 8t.
IIII 16 C. Sinice. Det chinefifche Turbao. $\mathrm{Sr}$
III2 17 C. Purpkrassens. Geve 1. 19. F. 190 19I. 2. b. 10 Si.

Turbo. Der Knopf.

1. Fauce fimplici.

A. Elevato granulati.

113 I T. Pice Das Riefenobr. Gmel Turbo pica. Sp. 39. Chema. 4. i. I51. f. J420. 1421 . \& S. 2. 176. \&. 1750 . Ii St.

1114 176 f. $1753 . \overline{3 \text { St. }}$ Chems. 5. 1.

$11152 \mathrm{~T}$. Sarmaricus Das orange Auge. Gmel. Turbo farmaticus. íp. 16. Chemo.

$1116 \quad 3$ T. Taiticus. Der Neufeelandifcbe fchwarre Knopf. Ginel. Turbo fasmaticus. If. 16. tebr feltea. 2 Sto

\section{Lade 5 I.}

11174 !T Smaragdinus. Der fmasagdfarbne Knopf Gmel. Turbo Imaragdus. Ip. 30. Chema. 5. t. 182. F. 1815. 1816. felteo. 4 St.

1118 5 ! T. Merula. Der Sehwarze Knopi. Gmel. Trochus finenfis, Ip 103. Chema. 5. 8. 165. f. 1564.1565 .3 St. 


\section{Letoratar}

III9|6 : T. Liibantrax. 'Der kohlchwarze. Knopf. Gmel. Trochus argyroftomus. Sp. 102. Chernn. 5. to 165. f. 1562. 1563. 2St.

11207 ! T. Aerbiops. Der Mohr. Wle Nr.6. nur genabelt U, kleiner. $2 \mathrm{St}$.

$11218 \mathrm{~T}$. Agreftis. Der kleine Tchwarze Koopf. Gmel. Trocbus sufticus, Tp. 35. Chemo. 5. 8. I70. f. 1645. 1646. 4 St.

I 1229 T. Teffellazus. Der gefprenkeite Knopt. Ginel. 'Trochus teflellatus. $T_{p}$ ' 106. Cbem. 5. to 166. \&. 1583 - 1587t IOSt

1123 10 T. Venofus. Der ausgedrechfelte Kaopfo I St.

I 124 II T. Punczulazus. Des geftippelte Knopk. I St.

112512 T. Neapolitanus. Der aeapolitanische Knopf, Ir St.

B Elevati fomplices.

II26 I2 *. T. Horticola. Det gebandete Knopf. I Se.

112712 * * U. T. Pileus, Der Huth - Knopf. Gmel. Helix pileus. 'P. 89. Cbeno 9. r. 122. f 1048 . I St.

$112812 * * *$ c. T. Sylveftris. Der umwrundene IKnopf. Gmel. Helix pileus. 'p. 89. Cbemn. 9. t. 122. \&. 1047. IS.

112912 d. T. Gialerus. Der gebandere Hutho Knopf. $2 \mathrm{St}$.

113012 e. T. Lineola. Der weirfe Hutb-Knopr. I St.

II3 I 12 f. T Globulus. Des Kugel-knoopf. Gmel. Helix cookiana. Ip. 230 . Cbemn. 9 ז. I22. f. 1049. 1050. I St.

113212 g. T. Iris.er Dunvergleichliche Knopf. Gmel. Trocbus iris ip. 86. Chemn, 5 . 8. $161.8 .1522 .1523 .7 \mathrm{St}$. 
88

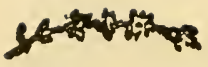

$1133 \mid 13$ T. Aurentiacus. Der bunts Knopf. 2 St.

113414 T. Granularus, Der granulirte Knopf. 8 St.

II 35 I5 T. Puftulatus. Der fehagrinirte Knopf. I St.

$113616 \mathrm{~T}$. Irregularis. Der überzukkerte Knopf. 1 St.

1137 I7! T. Granatum. Der Granat-Apfel. T. Martin. univ Conch, 2. f. 75 . I St

$113818 \mathrm{~T}$. Balaufinus. Der kleine Granac. Apfel. i St.

113919 ! T. Pulcberrimus. Der vortrefliche Knopf. I Se

II 4020 ! T. Perlatus. Der geperle Knopt. Gmel. Trochus diaphanus. Ip. 85. Chenn. 5. 1. 161. f. 1520. 152 \%. 3 St.

3411 2 I T Mugarzeceus. Det Scharfe Knopf. $\mathrm{ISt}$

II 4222 T. Uimbilicalis. Der genabelic Knopf. Lffer 632. f. 21 . Chemn. S. 1. 171. f. $1666.3 \mathrm{St}$.

114322 * T. Lutoreus. Der Holen Knopf. Gmel. Tuibo littorcus. 'p. 3. Chemn. 5. เ. 185. f. 185214 St.

$114422 * T$ Dourdis. Der kleine Knopf. Gnel. Turbolittoreus Sp. 3.r. Chem 5 . r. 585 , f. $185 ;$. a. b ISt.

II $4522 * * *$ T. Groenlandicus. Gmel. Turbo littoreus. Sp. 3.\%. Chemn.5. \$.185. 1855 . d. e.f.g. 2 St.

\section{Fauce perfonata.}

114723 T. Labeo Derfclackigte Knopf. Groel. Trochus labeo. Ip. 76. Chemn. 5. cab. 
c. 166. \& I579-8 ז. B.) Knorr I. t. Io.f.7. Geve t. I8. E. 165. a.6. 2 St.

$150 \quad \% \quad 2$ St.

$15 \mathrm{I}-$ 8. $\longrightarrow$ ISt.

$115224 \mathrm{~T}$. Labellum. Der grüne Knopf. I St.

I $5 3 2 \longdiv { 2 4 }$ * T. Sulcatus. Der gefurchte Knop\&. 4 St.

$154 \quad \%$ i St.

1 155 $25 \mathrm{~T}$. Tornatus. Der gedrecbfelec Knopf. Gmel. Trochusurbanus. 'p 48. Chemn. 5.t 171, f 1679. 2 St.

I is 526 T. Pharaonicus. Die Pharao.Schnecke. Gmel. Trochus pharaonis. fp.6. Cinems. 5. t. 17 1. f,1672.1673. Knorr I. t. 30. f. 6. \& 4. t. 26. f. 3. 4 . 3 St.

I 5727 T. Poripbareus. Der Kamifohlknopf. Ginel. Trochus pharonis. fp. 6. $2 \mathrm{Sc}$.

I I $5828 \mathrm{~T}$. Agyptiacus Der rothe Knopf. Gmel. Iroclius pbaronis. fp 6.Gever.I 3 . f 101 . a. b. I St.

I15929 T Subula. Der Hemdsknopl. Gmel. Trochus nodulus. ip. 50 . Chem. 5. ז. 171 . I I681. Geve ז. I3. ..104. a b. I St.

I I60 30 T. Senegalus. Der Knopf von Senegal. Ginel. Trochus guinenfis. ip. 49. Chemn. 5. I I71. f. I680. I St.

1 I6I 3 I T. Crenulatus. Der gereifre Knopf. Gmel. Trochus carneus. $\mathrm{p} .5$ I. Chemn.

II62 32 T. Elggans. Der zierlucbe Koopf. $2 \mathrm{St}$. 
8 C. Decollarum. Das abgeftumpfre weiffe Wickelkind. Ganel. Helix decol. lata fp. IIs. Chema, 9. c. 136. f. $1254.1255 .75 t$.

\section{Epitonium. Der Zapfen,}

I. Aperzura orbiculari. Mit Ziskel-

$$
\text { runder-Defoung. }
$$

I E. Scalare. Die echre Windeltrenpe. Gmel. Turbo fealaris. Ip. 62. Matr.4. t. IS 2. f. 1426. 27. I St.

2 E Breve. Die kurze Windeltreppe. Gmel Turbo fcalaris ip 62 . I St.

3 E. Listeatum. Die lineirteWindeltreppe. Gmel. Turbo Realaris. Tp. 62. r St.

4 E Medium Die geftreckte Windeltreppe. Gazel. Turbo fcalaris. Ip. 62. I St.

1178 S E. Principale. Die fein gerippre Win. deltreppe. Gmel Turbo fealatis. f́. 62. B. Martini 4.'t. 152, f. 1428.1429. 8 St.

II79 6 E. Vittazum. Die breitrippige Winde!treppe. Gmel, Turbo elathrus. โp. 6 \%. 5 St.

7 E. Clasbrus. Die gemeine Winde? creppe. Ginel. Turbo clathress if $\& 3$. Mart. 4. t. 153 f. 1434.1698.

I18r 8 E Elorgarstm. Die brăune fein gerippre Windeluseppe. Genel. Turbo clachrus. CP. 63. $3 \mathrm{St}_{\text {, }}$

1182 g E. Volentini. Die Valeutios. Windeltreppe. Gmel. Tuibo clashrus. Sp. $63 .^{6}$ Valentin sab. 1. fig.7. 2St. 
1183|10 E. Minutum. Die dünleiblgte Windel. rreppe. Gmel. Turbo olathrus. Sp. $63 ._{3}$ Martini 4. t. 155. f. 1435. 1436. 2 St.

\section{Lade 52.}

2. Aperure rotudata. Mit ründlicher Oefnung.

II E. Duplicarum Der doppelt gereifre Zapfen Gmel. Turbo duplicalus. Ip. 79. Martini 4. I I51. F. 1414. IISt.

I 8512 E. Acutarigulum. Det Ichatfwinklige zaplen. Gmel. Turbo acurangulus. 1p. 78. Martui 4. r. I5I. f. 1413. Knorr 3. 8. 19. f. 5. I Se.

$1186 / 3$ E. Dubium. Der biaune Zapfea. Gmel. Turbo replicatus. Sp. 77. Mas. tini 4. t. 151 . f. 1412 . Kootr 6. c. 25 f. 3 . 5 St.

1187 I 4 E. Terebra. Der Bohrzapfen. Gmel. Turbo terebra. fp 8r. Marrini 4. t :51. f. 1415 . Chemn. IO. t.165. f. 1591 . $5 \mathrm{St}$.

1188 is E. Tympanotonor. Det Trommel.Zapo fen. Gmel. Tuibo terebra. íp. 8I. Marcini 4. t. 151. \& 1416.9 St.

118916 E. Tornatunb. Der gedrechfelte: Z.apfen Gmel. Tu:bo terebi3. fp. 81. dArgenv rab. 14 fig. E. 3 St.

119017 E. Monocerns. Der dünfchaligte Zapfes Gincl. Turbo terebra. $\mathrm{fp}_{\mathrm{p}}$. 81. Marr. 4. t. 15 I. f. 1419 . $12 \frac{1}{2}$ Sr.

$119118 \mathrm{E}$. Infinitzum. Der Zapfen ohne Ende. Gmei. Turbo terebra, ip. 8 I 2 St. 
I192/19 E. Imbricatum. Der Senk - Zapfeo. Gimel. Turbo imbricatus. (p.76. Mar. tiai 4. to I52 f. 1422. t. 27. F. I. \& 6. t. 25. f. 2.3 St。

119320 ! E. Squamatum. Der Talchen-Zap. feo, Lifter 589. No. 53. I St.

I19.421 E. Rugofism. Der tunzligte Zspfen. Gmel. Turbo duplicatus, fp. 79. Mar. tini 4. t. 15 I. f. 1414 . J St.

119522 E. Varicgatum. Der braun und weirs gefickre Zapfen. Gmel. Turbo vieriegatus. 'p. 82. Martini 4. t. I52. f.I 423. Knotr 6. t. 16. f. $8.3 \mathrm{St}$.

119623 E. Margaritaceum. Der geperte Zap. fen. Gmel, Turbo variegarus. \{p. 82. var. 2 St.

119724 E. Brunneum. Der dunkelbrausue Zapfen. I St.

119825 E. Acuram. Det weife Charlgerillte Zapfen. Gmel. Turbo duplicstus. Tp.79. 1 St.

I19926 E. Marmorenm. Der gemarmelte Zap. fen. Gmel. Turbo imbricatus. Sp. 76. Martini 4. t. 152. f. 1422. 23. 7 Si.

$1200-\alpha$. 4 St.

120127 E. Eburneum. Der elfeabeinerne Zap. feo. 2 St.

120228 E. Vitreumn. Det gläferne Zapfen. $2 \mathrm{Sr}$. 120328 * 28. Fufillum Der kleine Zapfea.

3. Aperturs ooato-elongato eff $u$ sa.

Mit eysunder Oefoung.

120429 E. Maculatum. Das dicke Tiegerbein.

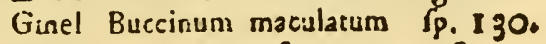
Matrini 4. t. 153. f. 1440. 7 St. 
1205130 E. Tigvinum. Das dünne Tiegelbeia. Gmel. Buccinum tigrinum. IP. 140. Martini 4 : 154 . . 448.3 St.

120631 E. Rubiginofum. Die szftige Penne. 2 St.

120732 E. Strintum. Die geftreife Penne. Gmel. Tusbo ftriatulus. is 67. I St.

120833 E Vittartun. Dic Kopfinadel. Ginel. Buccinum virtarum ip $134.3 \mathrm{St}$

120934 E Nova.Zcelendia. Die noufeelands Penne. 6 St.

$121035 \mathrm{E}$. Lafleunn. Die milchweifte Penne. Gunel. Turbo lacteus. Ip. 66 ISt.

36 E. Chalybeotum. Die Stah Mantel.

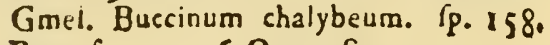
Rumpf. t. 29. f. Q. 3 St.

37 E. Lineatum. Die liniirte Penne. ise.

1214 38 E. Gurtncum. Die gefleckre Penne, Gmel. Bucrioum maeulatun \{p. $130 \%$ Martini 4. t. 153 . f. 14422 St.

I215 39 E. Dimidratum. Die orange Penne. Gmel. Buccinum dimidiatum. Ip. 138. Martini 4. 8.154 . f 1444.4 St

121640 E. Feldmanni Die Fildinaniche Penne. Gmel. Buccib. fubulatum Sp. 13 I. $\gamma$. Martion 4. t. 154 1446. $2 \mathrm{St}$

121741 E. Subularsin Die brauı gefleckre Penne. Gmel. Buccin。 fubulatum. Ip. 331. 4 St.

1218 a. - Marrini 4.t.153. f. $1441 \cdot 10$ St.

1219,42 E. Crenulatum. Die gekıòhute Pcoue. Gmel Bucen. crenulatum. ip. 132. Martini 4, t. 154. f. I445. 10 St. 


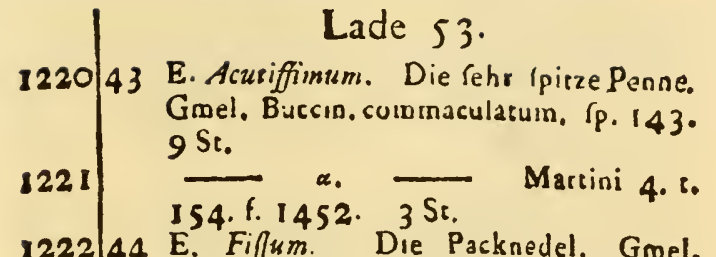

122244 E. Fiffum. Die Packnedel. Gmel. Buccin. Luccinctum. Tp. 142, Marsini 4. t. I54. f. I 45 I. $7 \mathrm{St}$.

122446 E. Avile. Diegelbbraune Penne. Gmel. Buccin. ftrigilatum. fp. 135. Martini 4. t. 155. f. I 456 . I St.

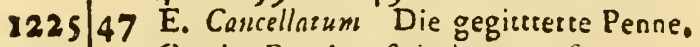
Gmel. Buccin. Arigilarum. Ip. 135. Martini 4.t. I55. f. I 456 . $2 \mathrm{St}$

122648 E. Pulcbellum. Die choüne fcheckigre Penne. $3 \mathrm{St}$.

122749 E. Minutum. Die kleine Penne. Gmel. Bucciu. haftacum. Ip. 144. Matriai 4.t. 154. f. $1454.3 \mathrm{St}$.

Plotia. Die halbe Penne.

1228 I P. Turrica. Die knobligte balbe Penne. I St.

12292 P. Lurida. Die fchwarzgraue halbe Penne. 2 St.

$1230 / 3$ P. Minuta. Die kleinliche halbe Penne.

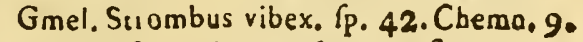
r. 136 f. 1261.1262 .2 Si.

12314 P. Fiscara. Die dunkelbraune halbe Penne. Chemn. 9. T. 135 . f. 1229. v. Born Tb. 16. Gg. 17. $2 \mathrm{St}$.

1232 5 P. Atra. Die fchwarzbianne halbe Pende. Gruel. Strombus arct. Sp. 39. Chemn, 9. 1. 135. 6. 1228; I Si.

12336 P. Fogilis. Die verkatkie halbe Penne. I Sr. 
$1234 / 7$ P. Dentaza. Die gezähnelte halbe Penne. $2 \mathrm{St}$

1235 8 P. Virginea. Die geftreckte Priuzew Flagge Gmel. Bulla virgineä. 'P. 24. Chemo. 9. t. 117 f. 1000.1003 . $5 \mathrm{Se}$

12369 P. PueHaris Die Prinzen Flagge. Gmel. Bulla virginea. Sp. 24 Chenin, 10.t 173. f. $1682-834$ St.

$123710 \mathrm{P}$ Lineete. Die linei. te halbe Penne. Gmel. Trochus dolabratus. 'P 113. Chemn. 5. t. 167. f. 1603. 1604. IOSt.

1238 II P. Filata Die umwunderre halbe Peone, Gmel. Trochus dolabratus. 'P. 113. 2 St.

1239 12 P. Puntiata. Die getippelte halbe Penne. Gmel. Trocbus dolabratus. fp. II3. I St.

1240 13 P Vistara. Die Kopf. Penne. Gmel. Buccinum viltarum. fp. $^{\text {134. Martini }}$ $4 \cdot t .155 \cdot$ f. 1462 . 12 St. - a. $^{4} \mathrm{St}$.

14 P. Tranquebarice. Die Penne. voa Tranquebar. Gmel. Buccin virıarum, IP I34. Martini 4. t. I55. E. J463. $4 \mathrm{St}$

1243 Is P. Dubia. Die Baftart-Pende. 3 St.

Strombus. Die Nadel.

I. Roftro recto. Mit grader Schaautze.

1244 I S. Paluftris. Der Loffel. Gmel Strom.

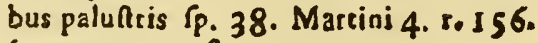
f. 1472.9 St.

Cehe felten. $2 \mathrm{St}$. 


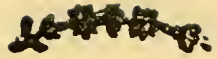

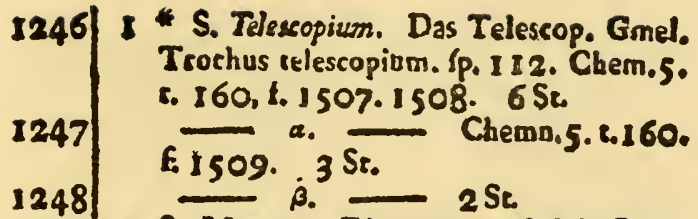

12492 S. Mangos. Die Mangos-Nadel Gmel. Mur.x molluccaous. fo 151 . Martini 4. t. 157. f. 1484 1485. Lifter Conch. r. 102 1. F 85 . I St.

1250 2 * S. Semiftriatus. Die geftreifre Na. del Gmel Murex molutcanus. 18 : 151. var. 2 St.

1251

2 * S. Tornetus. Die gedrechleite Na. del Gmel Marex radura. fp. 147. Fa. vanue t. XL. fig F. Schrôter Eunl. in Couch. 1. t. 3. . 67 St.

1252

*** S. Pifá. Der kleine Lôffel. Gmal. Murex cingulatus. If. 138. Martsi 4. r. 157. f. 1492. I Se.

12533 S. Aluco. Die Baftart Pabrktone. Gmel Murex aluco. Sp. I 34. Marr. 4. 4 I56. f. 1473-1477. 5 St.

$22544 \mathrm{~S}$ Bubo. Die ausgeknobbeire Nadel. Gmel Murex aluco. Ip. 134. vas. I St.

$12555 \mathrm{~S}$. Clave Herculis. Die Heskules. Keule. Gmel. Murex alueo. $\left\{p, 134 \cdot \gamma_{0}\right.$ Cbema. 10. t. 162. f. 1548 . 1549. 2 St.

1256 6 S. Muricatus. Die raube See-Nadel. Gmel. Murex aluco. Ip I34. Mars. 4. t. 156. f. 1475 . 3 St.

12577 S. Derticsldeus. Dis gazahoele N'sdel. Gael, Mirex aluso. ff. I39. Maro tini 4. t. 156. .. $147 \%$ 3 55

1258 S S. Granarius. Die gramulire Nadei. 2 Si.

1259 
126019 S. Sulcatus. Die geribte Nadel. 3 St. $126110 \mathrm{~S}$. Hexugcruss. Die vieleckigre Nadel. Gmel. Murex bexagonus, ip. 163. Chernn. 10. \&. 162. f. J554. I555. I St.

2. Roftro elevato. Mit erhabener

\section{Scbnautze.}

1262 Ir S. Nafurus. Die weiffe Schaeu.Peane. $2 \mathrm{St}$.

$12 \mathrm{~S}$ Vertagus. Die weiffe gefaitene Schneu Penge. Gmel. Murex vertagus. F. 133. Maltiol 4. t. I57. f. 1480 . 3 St.

13 S. Carminatas. Die bunte Schneu. Penne. Gmel. Murex vertagus. Ip. I 33. $\beta_{\text {. }}$ Martini 4. t. 157. f. 1482.3 St.

1266 if S. Fasctentus. Die gebandete ScbneuPenne. Gmel. Murex vertagus. If. I 33. $\beta$. Favanne Tb. 39. fig. C. I5. I St.

1267 I5 S. Gutsatus. Die gefleckte Schneu Penne. Gmel. Murex vestagus, '́p. 133. var. I St.

126816 S. Gramulatus. Die kornigre Schneu. Penne. Gmel. Murex granulatus. Ip. 149. Martini 4. t. 1 57. f. 14832 St.

126917 S. Cancellatus. Die gegitterte SchneuPenne. Gmei. Murtx granulatus. If. I 49. I Sr.

127018 S. Coronatus. Diegekrönte See-Nadel. Brand penne. Gmel. Murex aluco. Ip. 1340 Martiai 4. t. 156. f. 1478. 1479. 8 St. 


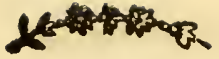

\section{Lade 54.}

19 S. Muricatus. Die ungefchliffene SeeNadel Gmel. Murex finenfis. Tp. 54. Martini 4 t. 157 . f. 1489.8 St.

127220 S. Anrulatus. Die geringelte See- Na. del. Martini 4. t. 157. f. 1486 . Mu. rex. Schrôters Eiol. in die Conch, I. P. 559.0.41. I Se.

3. Roftro eruncazo. Mit abgeftumpf-

$$
\text { ter Schnautze. }
$$

127321 S. Reticulatus. Die Atachligte See Na. del. Gmel. Murex fuenfis. Ip. 54. var. $5 \mathrm{St}$.

127422 S. Teftaceus. Die fchäckigte See-Na. del. Gmel Murex fordidus. 'p. 137. 2 St.

127523 S. Pellucidus. Die durcbfichrige See• Nadel. Gmel. Murex finenfis. SP. 54. var. 2 St.

1276 24 S. Perlatus. Die geperlte See-Nadel, Gmel. Murex finenfis. Ip. 54. var. I St.

127725 S. Ventricofus. Die bāuchigte Set. Na. del. Groel. Murex lordidus. Ip. I37. I St.

127826 S. Varicofus, Die warzigte See-Nadel. Gmel. Murex fordidus. [p. 137. Niate. 4. t. 157 . f. 1490.2 St.

127927 S Irreguleris. Die buntichäckigre See. Nadel. Gmel. Murex finenfis. fp. 137. I Se.

128022 * S. Fogitis. Die gefurchse See. Nab del. I St

128127 * S. Rofea. Die rofearotbe Sea-Nadel. 2 St.

1282 $27^{* * *}$ S. Bruned. Die braune See-N5del. I St. 
$1283 / 28$ S. Bolezus. Die Morchel. Gmel. Bucti. aamphallus. Ip. I46. Martioi 4.t. I55. f. 1464. 1465. Schröters Fluls Conch. P. 401.0 .194 . 5 St.

128428 ? S. Torrilis. Die ausgedrechfelre See- Nadel. I St.

Roftro Suberanceto. Mit verfchnitre. net Schoautze

128529 S. Spinofus. Die Sracbel-Nadel $3 \mathrm{St}_{0}$

$128630 \mathrm{~S}$. Turrssus. Die gethürmte See - Nà del. Gmel. Murex fluviatilis Ip. I 41. Lifter t 122. f. 18. \& 20. Favanne 39. Gig. C. 62 St.

128731 S. Nodofus, Die knorigre See - Nadel. I $S_{1}$

S. Pyremidalis Die Pysamide Gmel. Susombus aculeatus. Ip. 44. Clemn. 9. 1. 136 . f. 1267.1268 .4 St

128933 S. Babylonicus. Die babilonifsbe See Nadel ISt.

4. Rofro obfoleso. Mit kurzes Schantze.

$1290 / 34$ S. Obrufus. Die Atuonfe See-Nadel. Gonel. Serombus ausicus. Sp. 43. Favan. ge Tb. 39. fig. C. 19 3 Si.

1291/35 S Canalizulariș. Vie Knopf-Nadel, Gmel. Strombus lividus. Yp. 49. Chemn. 9 t. 136. f. 1269.1270 .2 St.

129236 S. Porcellanus Gmel. Strombus auricus. Ep. 43. Littet Conch. 1. 121 . f. 16 . 6 Si.

129336 a - a. Chemn. 9. i, 136. f. 1265.1266 .3 St. 129437 "' S Discoideus. Die Scheiben-Nadel. 


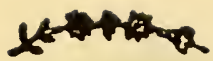

101

Schuóters Einl. io die Concb. T.p. 561 .

n. 47. Favanoe Tb. XL. Gg. IK. Lifter

c. 120. f. Is. ISt.

Lunatica. Das Knobbelhorn oder

die Mond-Schnecke.

I L. Maxima. Das groffe Knobbelhorn. Gmel. Turbo olearius. Ip. 17. I Se.

2 L. Viridune. Das grüne fchäckigre Knobbethosn. Gmel, Turbo olcarius. ip. 17. var. y. Chemn. 5. t. 178. f. 1773.1774 I St.

3 L Uudulaza Das wellenförmige Knob. belhorn. Grel. Turbo marmoratus. Ip. 15. Chemn. 5. t. 181. f. 1775 . 1776. 2 St.

4 L Marmorasa Das Schlaogeafell. Gmel Turbo inarmeratus. fp. I5. $5 \mathrm{Se}$.

5 L. Aruginofa Der grüne Naftaues. Chemn 5:183. F. 1833 . I St

5 * 2 Siluck dico, woraut Blu. meo gefehoitrtn. 2 St.

5 * L. Imperialis. Der kayferliche Sile bermund. Groel. Turbo imperialis. Sp. 20. Chemn. 5.6. 180. f. 1790. IS.

6 L. Sirmea. Der gedömere Silbermund. Gonel Turbo cornusus. \{p. 18. Cbemn. S. 5. 179. E. 1779. 3780 . $6 \mathrm{Se}$.

\section{Lade 55.}

13037 L. Cbryfofrome. Der Golumund. Amel. Tuibo chryforomus. $\mathrm{f}_{\hat{\mathrm{y}}}$. 10. Chemn. 5 . 1 178. 6. 1766. Koors Vesg. 2. t. 14. f. 2. 5.2 .13 1. 3. 35 . 
1304 7 L. Cbryfofeome, a. 5 St.

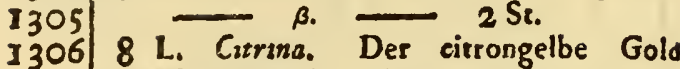
Mund. Gmel. Turbo chrytoftomus. Ip. IO I St.

9 L. Spinofa. Der Atacbligte Goldmund Gmel. Turbo fpinofus. Sp. 24. Chemo 5.t. 181 . f. 1797. 4 Sr.

1308 10 L. Granulata. Der granulirte Silber. Mund. Gmel. Turbo crenulatus. fp. 29. Chemn 5.t 182, f. I81 1. 12. 2 St

J309 II L. Tuverculata Det warzigte Silbes Mund, Gmel. Turbo Molckianus P. 26 Chemi. 5. t. I8I f. 1799. 1800. 2 Se.

1310 12 L Argyrofroma, Det geteifte Silbet. Mund Geve t. 17. f. $160.3 \mathrm{St}$

13 IJ 13 L. Argentata. Der glatre Silbermuad. Gmel, Turbo canalicularus. Sp. 22. Chemn. 5. t. 18 I. T. 1794 iSt.

131214 L. Maculata. Der gefleckte Silber. Mund. Geve t. 17. f. 159. 2. 159. b. \& 16 . 5 St.

1313 is L. Taiterzifs. Det othabeitifche SilberMund. 4 St.

$131416 \mathrm{~L}$. Verleulnm. Die fchwarze Bürfte. Gmel. Turbo fetofus. \{p. 23. Chemn. 5 . t. 181. f. 1795.1796 .2 St.

I3I5 17! L. Brunea. Der fchwarzbraune SilbetMund. Gmel. Turbo argyroftomus. Ip. 41. Chemn. 5. t. 177. f. 1764. I St.

$1316-\alpha$.

$131718 \mathrm{~L}$. Pita. Det buntfchäckigte SilberMund. Gmel. Turbo argyroftomus. Sp. 41. Chemo. 5. t. 177. f. 1763. I St.

131819 L. Squanofa. Der fchuppigre Silber. Mund. Gmel. Turbo argyroftomus. fp. 41. Chemn, 5. C. 177, f. 1764. 5 St. 


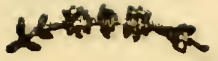

$131920 \mathrm{~L}$. Obscura: Der disakelbraune $N_{a}$.

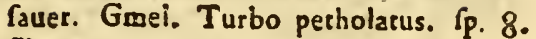
Chemn. 5. t. 183. f. 1830.31. I St.

132021 ! L Marmorate. Der fchö́n marmo. ritte Naffauer. Gmel. Turbo perbola.

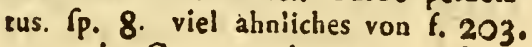
c. 20, im Geve, nur brauner. I St.

132 I 22 L. Porpbyria. Der Porphir-Naffauer. Gmel. Turbo petholatus. Tp 8. Chemn. 5. t. 184 . f. 1838 I St.

132223 L. Cinguleta. Der gegürtelte Nađauer.

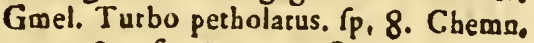
5.t. 183 f. 1832.2 St.

132324 ! L. Sericee. Der kleine fehône Naffauer. Gmel. Turbo petholatus. Sp. 8. I St.

I324 25 L. Destigrata Det fchwärzliche $\mathrm{Naf}$. faner. Gmel. Turbo petholacus. Sp. 8. Cbemn. 5. t. 183. C 1830. 1831. I St.

132526 L. Petbolata. Der giatte bandirte und marmoritte Naflauer. Gmel. Tusbo petholazus. fp. 8. I St.

f. $1828.1829 .13 \mathrm{St}$.
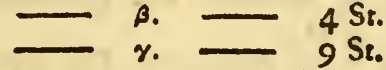

- 8. Chemn. 5.t. 183.

f. $1834 \cdot 1835 \cdot 4 \mathrm{St}$.

\section{Lade 56.}

Lunella. Die Mond-Schnecke.

$$
\text { I. Spira eleoata. }
$$

13301 L. Viridana. Die Atachlige Mondfchn. Gmel. Turto coronata Ip. 2 I. Chemn. 5. t. 180 . f $1791-1793$. 2 St. 
$1332 \mid 2$ L. Moniliformis. Die knotige Mond. fchnecke Gmel. Turbo granulatus. Sp. SI. B. Chemn. 5. 1. 176. I 1746. 3 St.

13333 L. Appendiculate. Die geftachelte Mond. fchnecke. Chemn. 5. 2. 171. F. 1665. 2 St.

1334 4 L. Ciduroides. Die geruuzelte Mondfchnecke. Cbeain. 5. 165. f. 1567. $682 \mathrm{St}$.

13355 L. Anomala. Die wulnige MondfchnGmel. Turbo cidaris. Ip. 34. viridis. Chemn. 5. ז. 184. f. 1842. 1843. 2 St.

6 L. Moslemica Die Enirs-Mondrchn. Gmot. Turbo cidaris. ' $p$. 34: Chemn. 5. . 184. f. 1842 . 7 Se.

2. Spira consigua.

7 L Piđaa. Die buntehâckigre Mond. fchnecke. Gmel. Turboverficolor. Ip 43. Clienin. 5. t. 176. f. 1740.1741. $3 \mathrm{St}$.

8 L. Striaza. Die gebandere Mondfehn. 2 Se.

13398 * L. Pulverata. Dic gepuderte MondScbnecke ISt.

13409 L. Cruenta Die mit Blut geficckte Mondfehnecke. $2 \mathrm{St}$.

$134110 \mathrm{~L}$ Lurcola Die geibe Mondfchnorke. Gmel. Turbo neracoides. Tp. 2 Chemn. 5. 1.185 f. 18541855 . 2 St.

f. 1854 . fig. 9. 10. $2 \mathrm{St}$

1343 II L Ncritrides. Die neritenförmige Mondfchnerke Gmel. Tuibo obrularus. fp. 1. Cheinn. 5. 1. 185 . f. 1854 . fige. 2 St. 
1344/12 L. Vitellata. Die weirs - und gelbgebandete Mondfchnecke. Gmel. Turuo neritoides. $\mathrm{r}_{\mathrm{P} .} 2$ Chemn. 5. i. 185. f. 1854. fig. 3. I St.

$134513 \mathrm{~L}$ Radula. Diefcharfe Mondfchnecke. Gmel. Nerita radula \{́. 27. Chemo. 5. ז. 190. 1946.1947 .3 St.

\section{Lade 57.}

pythia. Die Zauberfchnecke.

I P. Helicina. Die Hexe Gmel. Helir Scarabeus. If. 1. Chemn 9. t. 136. f. 124.9. 1250. Knorr Verg. 6. 1. 19. f. 2. 3. $6 \mathrm{St}$.

Ellobium. Das Midas-Ohs.

s. Cylindracea.

1347 I E Midre. Das grote Midas.Ohe. Gmel. Volura auris midx. Ip. I abge: fchliffeo. Mart. 2. t. 43 . f. 436.437. I St.

2 E. Ceramenfe Dasgrofre dünntchalige Midas Ohr. Gmel Voluta auris midx. Ip. I Martini 2.t 43. f. 438 . I St.

3 E. Tumidum Das dickbauchigte Mi. das Oht. Gmel. Voluca auris mida $r_{p}$. $f$. i Sr.

J350 $4 \mathrm{E}$ Labrofirm. Das kleine Midas Ohr. Gmel. Volura auris judx Ip. 10. Mart. 2. 1. 44 T. 449.450 .3 St.

5 E. Subrile. Das oleine dünnfchalige Midas. Olir. Gmel. Voluta auris judx. Sp. 10. Mauni 2.t. 4 4.f. 45 1. I St. 
13526 E. Ivflnmmarum: Das gebandere Mi. das Ohr. Gmel. Volura coffea. $\{p .15$. Lifter t. 834. f. 59. Chemn 9. t. I2 I.I f 1043.44 .2 St.

7 E. Barbedenfe Das Midas-Obr von Barbados, Gmel Voluta coffea. Ip. is. Matrini 2. t. 43. f. 445 . I St.

\section{Buccinotdea.}

8: E Aufrale Das peufeeländféhe $M i$. das Ohr. Gmel. Voluea auris Malchi. fp. I1. Selten, Chema. 9. I I2I. f. 1039. 1040: 1 Si.

9 E. Flammerm. Das bunte Midas Ohr. Gmel. Voluta glabra. Sp. 8. Mattini 2. t. 43 . £. $447 \cdot 448$. 4 St.

1356 10 E. Ceriona. Das fehneeweille MidasOhr. Gmel Helix labiofa. fp. IOI. Chemn 9. r. 135 . f. 1234.2 St.

135711 E. Virgineum. Das blasrothe Midas. Ohir. Gmel. Helix pudica. rp. 102. Chemn. 9 t. 121 . f. 1042. I Sh

Helix. Die Schraube.

I H. Hepatica. Die braune Schraube. Gmel. Bulla falciara fp. 25. I St.

$2 \mathrm{H}, \mathrm{Tefa} \cdot$ ovi Die weifte Schraube. Gmel. Bulla fatciata $f_{p .} 252 \mathrm{St}$,

3 H. Fesciera. Die Staaten. Flagge. Gmel. Bulla farciata ip 25 Chemn.9. t. 117. f. 1004-1006. 4 St.

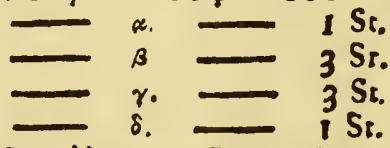

4 H. Oblonga. Das falfche Midas. Oht Rofenmund. Gmel. Helix oblonga, 亿p. 87. Chemn, 9. t. I19. f. IO22. I023. $4 \mathrm{St}$. 


\section{4tata}

1366 H. Globulus. Das kleine dünofebalige

1367 Falfchre Midas.Obr. 2 St.

13676 H. Fulice. Die banre Henne. Selten. I St.

$13687 \mathrm{H}$. Ellobioides, Das geftreckre Midas. Ohr. ISt.

$13698 \mathrm{H}$. Rallhus. Der bunce Haho. Gmel. Bulla Zebra. Sp. 31. Chemn. 9. 8. I18. f. $1015.1016 .85 \mathrm{St}$

3370

H. Tumida. Die kleine bauchigie Schraube. Gmel. Bulla derrita. Sp. 139. I St.

$137110 \mathrm{H}$. Elongata. Die lang geftreckte Schraube. Gmel. Helix detrita. Ip. 139. Chemn. 9. t. 134. f. 1225. a. ISe.

1372 Is H. Ligasa. Die gebandere Schraube. I St.

$137312 \mathrm{H}$. Brunuea. Die brauolich gebandete Schraube. I St.

1374 I 3 H. Albida. Die Tchmutzig weife Schrabbe. I Sc.

3375 1.4 H. Carmate. Die buntfcbāckige Schr. ise. a. I St.

I5 H. Detrisa. Die abgefobliffene Schr. Gmel. Helix detrita. 'p. 139. Cbemn. 2. t. 134. f. lit. c. d. 4 St.

$1378-2$ St.

1379 - B. links gewunden Seleen i St.

138016 H. Biliofa. Die vielfarbige links Scbr, Gmel. Helix perverfa. $\int_{p} .94 . \gamma$. Telten. Chemn. 9. t. Iro. f. 932.933. 2 St. $1381 \frac{210.8}{110.836 .937 .}$. St.

1382 I 7 H. Citrina. Die cirronengelbe rechre Schraube. I St. 
$1383 \mid 18 \mathrm{H}$. Dextra Die citronenfarbede Schraube. Gmel. Helix dexrsa. Ip 25. Cheinn. 9. c. I34. f. 1210.1211. 2 St.

$191 \mathrm{H}$ Perverfa Die linksgewundene fchwefelgelbe Schraute. Gmel. Helix perveifa. fo. 94. Felien. $\beta$. Chemn. 9. t. 110 . f. 934.935 . I Sr.

1386 a. - ganz weiffe. 2 St.

$138720 \mathrm{H}$. Inverfa. Die Areifigte Schraube. Gmel Helix inverfa. Cp. 97. Chemn 9. 1 110. f. $925.926 .6 \mathrm{St}$. $\mathrm{H} \longrightarrow$ \&. I St.

$21 \mathrm{H}$. Trfacer. Die caftanienbraune

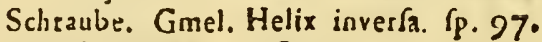
var. Sehr felten. I Se.

139022 H. Lava. Die umwundene Schraube. Gmel Helix lava. Sp 300 . Felten. Chemn. 9. t. II I. f. 946. 947. 2 St.

$139123 \mathrm{H}$. Interrupza. Die unterbrochne Scluraube Gmel. Helix contraria. Sp. 99. felten. Chemn. 9. t. II 1. f. 938.939. I Se

$139224 \mathrm{H}$. Speciofn Die vorzüglich fchone Schraube. Gmel Helix contıasıa 〔p. 99. relten. I St.

$139325 \mathrm{H}$. Liferi, Die Lifterfche Schraube. Gmel. Helix columna fp. 122 auffert felten. Lifter Conch. r. 38. f. $37 \& 39$. Chemn. 9. t. 112 . f. 9 i4 955 . 1 Sr.

$139426 \mathrm{H}$ Uftilago. Dic unarchte gefreckte Schraube. Gmel. Helix flammea 1 . 88. Cbemo. 9 t. I I.9. I 1024 25. ISt. $139527 \mathrm{H}$ Pbafiamus. Der Phafan Ginel.Buccinum aufrale. Ip. 173. Felten. Chemn. 9.t. 120 f. $1033.1034 .2 \mathrm{St}_{\text {. }}$ 


\section{Letor}

J396|27* H Variegato. Die gefprenkelie Schraube. 2 St.

139728 H Pulverulensa. Die gepuderte Schr. I St.

139829 H. Stagnalis Die dünne Flufscthraube. Spizzhorn. Gmel. Helix Itagnalis. ŕp 128. Chemn. 9 t. 135. f. 1237.1238. 5 St.

$139930 \mathrm{H}$ Fraglis. Die durcbfichrige Flurs. Seliraube. Gmel. Helix ftagnalis, Ip. 128. 2 Sc

1400 3 I H Faluftris. Die beaunnündige Schr. Gmel Heliz Rugnalis. fp. 128. Marsini 1239. 1240. 6 Ss.

$140132 \mathrm{H}$ Vivipara Die Rumpfe Scliranbe. Gmel Helix vivipara. Íp. IO5. B. Clientn. $91132 \& 1182$ II $83.5 \mathrm{St}$

$140233 \mathrm{H}$ Angaria. Die Hornlcheraube. Gmel. Turbo lincina P. 7 I. Chenio.9.8.123. f 1060 a. I St.

$340334 \mathrm{H}$. Butcimulum Die kleine gelbe Sctraube Gmel. Turbo lincina. Ifp. 7 I. Martui 1060. b. c. 2 St.

Thara. Die dreyfache Krone.

1404 I T. Amarrila. Die Rivier Pabft Krone. Gmel. Helix amarula. Sp. 126. Cbemn. 9 t. 134. t. 1218.1219. Rumpf t. 33 f. F.F. 7 St.

1405

$2 \mathrm{~T}$ Cancellata. Die gegitcerte Riviet Pablt-Kione. Gmel. Helix amarula. IP 126. * Chemn. 9. 2. 134 f. 1220. 1221 . I St.

3 T. Eximt6. Die lang geftreckte Rivies $\mathrm{Pab}$ ) $\mathrm{K}$ orre. Sebr fciren. I St.

1407 4 T. Fraftans Die gelbbraune Rivies Pabrt Kione. Tehr felcen. I St. 1408
$5 \mathrm{~T}$ Minuta. Die kleiwe Pabft. Krone.
3 St. 
110 14.tox

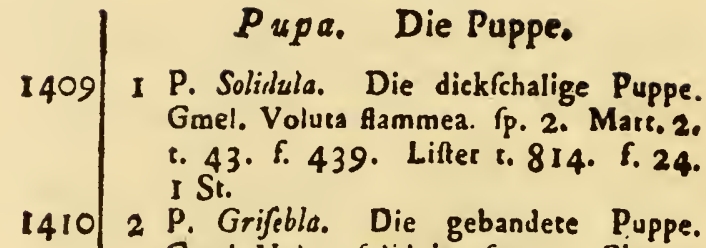
Gmel. Voluta folidula. If. I3. Chema. 10. t. 149. f. 1405. 2 St.

\section{Oleacina. Die Olive.}

I O. Volusara, Die Olive. Gmol. Bulla volura 'p. 40. Chemn. 9. B. 117. f. 1009. 1010 . ISt.

\section{Lade 58.}

Ampulla. Der Wafferkring.

I A. Aibatina. Das sothe Rebhubn. Gnel. Bulla achatina. ip. 32 ß. Chemn 9. t. 118. f. $1012.1013 \mathrm{~s} 5$.

2 A. Fianmea, Der geflaminie Warter. krug. Gmei. Bulla achatina. \{p. 32. 2 St.

3 ! A. Bombarda Der kugeiiormige Walferkrug. Gmel. Bulla achatina. Sp. 32. I St.

4 A. Lactere. Der milchweifre Wafferkrug. Gincl. Bulla acharina Sp. 32 I St.

5 A. Purpures Der purpusfarbne War. ferkrug. Gmel. Bulla achatin 3. 'p. 32. Matrini 1017.1018. 3St.

$14186 \mathrm{~A}$. Kambenl. Der geglectere Wafferkrug. Gmel. Bulla Zehra íp. 31 Martini 1024 1025. Lifter 578. f. 33 . 9 Si. 


\section{6.}

1419 7 A Zebre. Der grofte Kapiche Efel. Gmel. Bulla Zebra. Ip 3 1. . Chema 9. t. 118. f. $1014.7 \mathrm{St}$.

8 A. Quagga. Des geftreckte Zebra. Gmel. Bulla Zebra fp. 3I. I Sr.

9 !! A. Priamas. Det Priamus. Gmel. Bulla Stercus pulicum, (p. 45. Chem. 9. t. I20. f. 1026. 1027. I St.

\section{Lade 59.}

Buccinum. Das Kink-Horn.

1423 B. Undatum. Das welleoformige Kink. Horn. Gmel. Buccinum undacune. \{p, 93. $2 \mathrm{St}$.

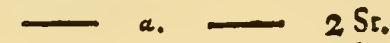

$\beta_{0} \longrightarrow 2 \mathrm{St}$

f. $1206-1210.1$ St.

2 B. Tumidum. Das bauchige Kink-Horn. 2 St.

3 ! B Elongasum. Das dünne aufgeblafene Kink. Horn mit 9 Windungen, relteo. I St.

4 B. Anglicrm. Das eoglifche Kiok. Horn. Gnel. Buccinum vulgatum. fp. 120. Mastini 4. t. 124. f. I 162. $1163.15 \mathrm{~S}$.

5 B. Porcatsm. Das gefurchte Kink. Horn. Gmel. Buccinum vulgatum. $\int_{p} .120$. 3 Marsini 4. ¿. 124. f.1165.1166. 2 St.

14315 * B. Dorfatum. Der Schilduäger. Gmel. Buccinum vulgarum. '́p. 120. Már. sini 4. c. 325 . F. 1194.95 .3 St.

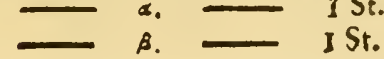


1434| 6 B. Quadridenticuletum. Das 42ahnige Kink. Hurn. Gmel. Buccinum vulgarum. fp. 120 var I St

14356 * B. Politum Das glatte Kiuk. Horn. Gmel. Bucsinum vulgatum. Ip. 120. I St.

14367 B. Dubiues. Das gefalzene Kink-Horn. eod. I. St.

14377 * B. Caffiarum. Das Helmförmige Kink. bord. eod ISt.

1438 ist.

14398 B. Cbryfoftomum. Das kleıne gegitterte Kink-Horn. Llfter 966. F. 21. eod. I St.

14409 B. Granulare. Das körnigce Kink. Horn. eod ISt.

144110 B. Striasum. Das geftreifre Kink.Hora. I St.

3442 a. 2 St.

J443 I B. Consortum. Die linksgedrehte Harfe, Sebi felten. I St.

144412 B Glaciale Das Kink Horn des Eis. meets Gmet. Buccinumglaciale. \{p:92. Cbemn, 10. 1. 152. f. 1446. 1447. 3 St.

144513 B. Islandicuin. Das sslâodifche Kink. Gmel. Buccinum indicum, Ip. 114. Martins 4 t. 123. f. 11381139. 2 St.

144614 B. Limeatum Das linirte Kink. Horn. Gmel. Buccinum glans. '́p. 41. Maro tini 6. t. 125 . f. $1196-97$. S S.

144714 * B Faba. Die wesfe Bohne, ISt.

144815 !! B. Turgadum. Das vielpuntirte Kink. Horn. Gmel. Buccinum turgidum. Tp. 184. Chemn. 10. t. 154. f. 1475. I476. Maityn uoiv. Conch. 2. t. 49. d. relten ist.

144916 B. Flommigerum. Das gezackie Kiok. Horn. J St. 


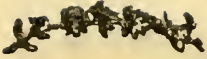

1450/17 B. Crreum. Das wachsgelbe KinkHoro, ISt.

$145117^{*}$ !! B. Fereftratum Das groffe gegitertse Kink.Hora, fehr felten. I Sr.

145218 B. Cinftum. Das bohlkehlige Kink. Horo. Gmel. Buccinum porcatum. \{p. 105. Martioi 4. t. 126. f. I213. 1214 Kuors, 6. t. 33. f. 3. $2 \mathrm{~S}_{2}$

145419 B. Britancum. Das britrifche Kink. Horn Gmel. Buccinum. anglicum. fp. 104. Martini 4. t. 126.f. 1212. I St.

145519 * B. Scalare. Die Treppe. Gmel. Buccinum anglicum. Tp. 104. Selten. I St.

145620 B. Excavatum. Das ausgehobelte KinkHorn. Gmel. Buccinum anglicum. Sp. 104. 4 St.

$1457={ }_{1}={ }_{1}={ }_{1}$ St.

145920 * B. Impreffum. Das eingedruckce Kink. Horn I St.

1460 21 B Rufescens. Das buntrcbāckigre Kink. Hopn. Gmel. Buccinum poiss. tum. Y p. 105. $2 \mathrm{St}$.

146122 B. Infarum. Das weirsgekehlte Kink. Horn. I St.

146223 ! B. Lamellofum. Das b!ättrige braune Kink Horn, Murex Aufralafiz, aus der Südfee. 2 St.

1463 - u. Tehr felten. 2 Si.

146424 B. Papillofurn. Der Reisbrey. Gmo!. Buccinum papillofurn. If 40. Mazr. 4. t. 125. f. 1204, 1205. Kaors 3. \&. 27.6 .2 .2 St.

I 46525 B. Purctulatim. Das punctiste ISinkHorn, I St. 


\section{comprover}

1466/26 B. Diapbonum. Das klare Kink Horn. Gmel. Buccioum textum, Ip 102. Mat. tini 4. 2. 125. f. 1201. 1202 . ISt.

146727 B. Comellinum. Das geripie KiokHorn. I Se.

146828 B. Senticofum. Das Dieftel . Horn. Gmel. Morex fenticnfus. 'p. 49. Martini 4. t. I5s. f. 1466. 1467. Knorr 4. c. 23.54 .5 .5 St.

146929 B. Caffidoideum. Das gegürcelte Kink. Hora. I Se.

$1470 / 30$ B. Lamboides. Das susgefchaitcene Kiok. Hora. 2 St.

I47 I 3 I L Laigarum. Das geglättete Kink. Gmel. Bucciaum lavigasum. 'p. 129. Martioi \&. t. 125. f. 1214.1216. 2 St.

147332 B. Porphyrosdes. Das zugefpitzte KiokHoro. 5 St.

147433 B. Obrufurn. Das fiumpfe Kink - Horn. 2 S:

147534 B. Cingularum. Die kleioe zeyloofthe Dauel. Mastioi 3. f. 564. 3 St.

I476 35 B. Glabratum. Die zeyloofebe Datrel. Gmel. Buccinum glabratum. 'p. 81. Lifter t. 974. f. 29. Mart. 4. 122. f. $1117.6 \mathrm{Si}$.

1477

3478

36 B. Spirarum. Die genabelte Fifcbreuse. Gmel. Buccioum Tpiratum. Tp. 70. Martini 4. ?. 122. ह. 1118. Kooss 2. B. 6. t. 5. \& 3. 8. 3. f. 4.10 St.

148137 B. Girarum. Die gezäbrelte Filchreufe. Gmel. Buccioum glabratum. fp. 81. 6. Metrini 4. 8. I22. fo II19. $5 \mathrm{Si}$. 


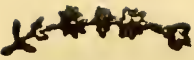

$1482 / 38$ B. Meculofwm. Die gefleckte Fifh. reufe. Gmel. Buccioum Spiratum. fp. 70. B. Martioi 4. t. 122. E. I120. $1121.3 \mathrm{SL}$

148339 B. Rude. Das plumpe Kinkhorn. Gmel.

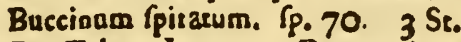

148440 B. Tubersularum. Das geknobbelce Kiakborn, ISt,

\section{Lade 60.}

Neptunea, Das Nepeunus.Horn.

I N. Despecia. Das islāndifebe Nepcus tanus. Horn. Gmel. Murex antiquus. Sp. 73. Martioi 4. 2. 138. f. 1296. $6 \mathrm{Sc}$.

2 N. Neglecta. Das nordifebe Nepisous - Horn. Gmel. Murex sntiquus. Tp. 73. vat. Mart. 4. t. 138. f. I295. 2 St.

3 N. Limbass. Das gefiumre oordifche Neptunus. Horn. Gmel. Murex anti. quus. Ip. 73. 3 St.

6. I291. 3 Se.

14884 N. Ansiqua. Das fein gegitterte nosdifehe Neptuaus-Horn. Gmel. Murex antiqusus. fp. 73. Marsini 4. 1. 138. f. 1294. $8 \mathrm{St}$.

1489

51 N. Contraria. Das linksgewandese nord:Sche Neperuaus. Hoto. Gmel. Mís. rex contrarius. Ip. I57. Martini 9. t. 105. f. 894. 896. Regeafurs Cotzh. 2. 5. 4. E. 36 . I St,

14906 I N. Perver $\sqrt{a}$. Das linksgarundene dickfchalige nordifche Nieptunus. Hosn. I St. 
1491/ 7 N. Poxderofa. Das braunangelaufeae nordiche Nepunus-Horn, I St.

8 ! N. Corona Mexicana. Die Mexicanifele Krone. Gmel. Murex curona, Ip. 161. auffer it felten. Chemn, I0. r. I6I. f. 1526. 1527. Davil. catal. raif, I. t. 9. E. A. I St.

$14939 \mathrm{~N}$. Clorbrus. Das blaturige nordifche Neperunus-Hora, felcen, Kammerert. 9. 6. 2. 2 St.

10 ! N. Foliaces Der Granat - Apfel. Ginel. Mutex magelanicus. (́p. 80. Martinl 4. t. I 39. f. I 297. Koott 4. t. 30. f. $^{2} .4 \mathrm{St}$.

1496 Io * N. Muricina. Dasfein geknobbelte nordifche Nepeunus. Horn. I St

149710 * N. Canaliculaza. Das ausgedrehre nordifehe Neptuuns. Horn, I St.

\$4981I N. Cancellata. Das Gitrerwerh. I St.

149912 N. Reticulara. Das Netz. 2 St.

$150013 !$ IN. Magellarica. Vie gegitrette Schnecke aus Neufeeland. Gmel. Murex magelanitus. fp. 80. $\beta$. felten. Chemb. 10. t. 164. f. 1570 . I St.

$15011_{4}$ IV. Varicoja. Die wulfige Schnecke. Gmel. Murez subecula. Ip. 35. Chemn. 10. t. 162. .. $1546.1547 .3 \mathrm{St}$.

1502114 * N. Foffilis. Die ausgegrabene Scha. I $S$.

150315 N. Piffilla. Das kleine weife Gitterwerk. Gnisl. Buccisum niveum. Sp. I I 2. Martini 4. t. 122. f. II22. 1123. 3 Se.

$150416 \mathrm{~N}$. Doliata. Das geringelte Neptunus-

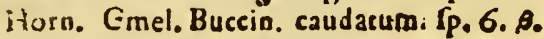
Eifter Conch. t. 940. f. 36.4 St.

150517 N. Nafara. Dit zugefpitzre Schnecke. $2 \mathrm{~S}$. 


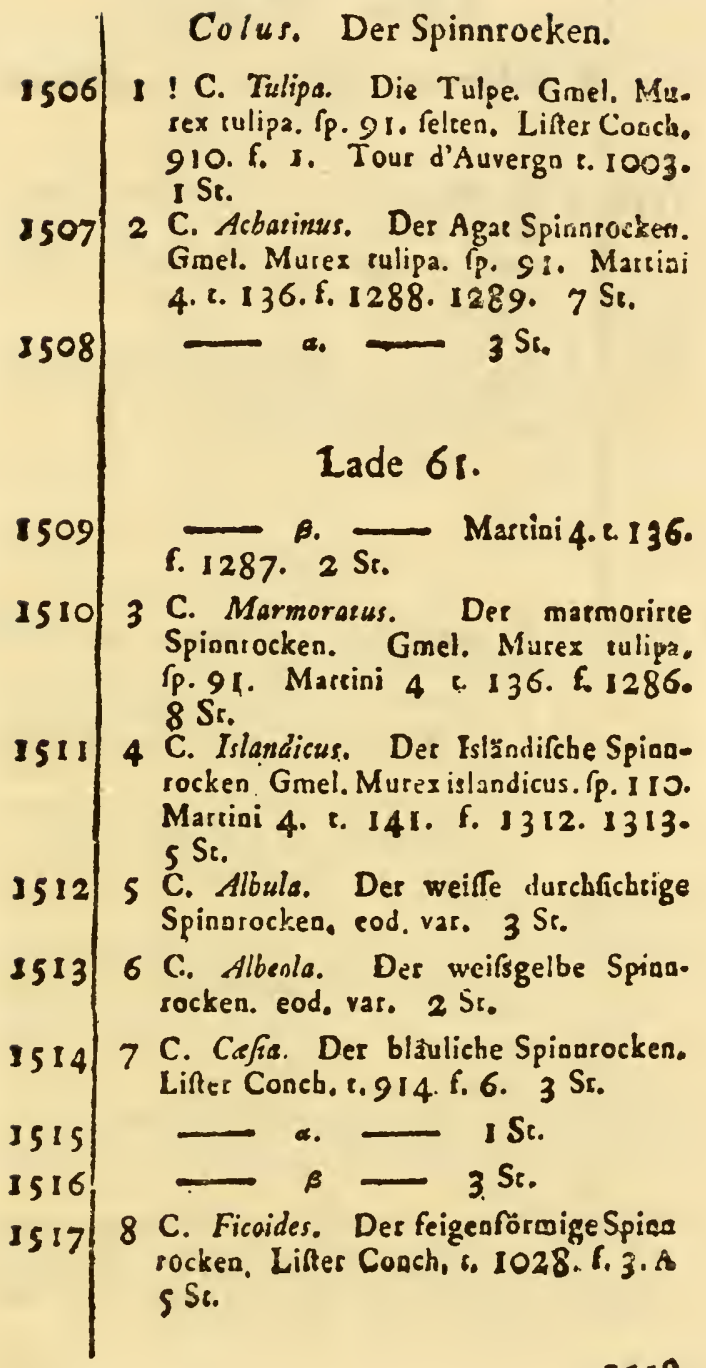


F usus. Die Spindel.

1. Columella plicara. Mit gefalteter Aebfe.

I518 I F. Trapezium. Das Perfilche Kleid. Gmel. Murex trapezium. (p. 99. Mar. tiai 4. t. 139. fo 1298. 1299.8 St.

1539202 St.

2 E. Cynara. Die Artifchocke. Gmel. Murex feolymus. '́p. 101. Marrini 4. c. 142 . f. 1325.4 St.

\section{Lade 62.}

15223 F. Cydonium. Die Quitte. eod. var. relien. I Se.

I523 4 F. Simplex. Die glatte Spindel. 4 Se. 4 F. Scrupeus. Die warzige Spindel. I St.

15245 F. Filamentofus. Die geringele Spio-

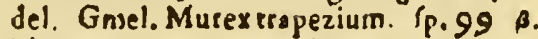
Marrini 4. t. 140 f. $1310.131 \%$ $12 \mathrm{Se}$

1525 $6 \mathrm{~F}$ Folygonus. Die vielwinklige Spin. del. Giriel Murex polygorius ip. 109. Maptins 4. t. 140. f. I306 1307. Knair 5.1 I0. f. 4. 6. 6. 15. f. 5. 5 St.

15266 * F. Polyedros. Die vielwinklige Spindel. Cmel. Murex folygonus. fp. 109. I St.

$15276 *$ F. Angulatus. Die wultige Spindel Gmel. Murex polygonus. is 109. Mastuai \& t. 141.6.1314. 15. 15 t. 
I528 6 *** F. Fasciatus. Die weifgebandete Spindel. Gmel. Murex undularus. $\{$ p. I 73. Lifter Conch. 92 I. f. I4. a. Chemn. 10. c. 162. E. 142. 43. Cab. Rudolit. p. 138. n. 5. t. 10. f. I. 2 Se.

1529

7 F. Glaucus. Die blauliche Spindel. 4 St.

8 F. Minutus. Die klcinliche Spindel. Marsini 4. to 141. f. 1121.222. 4 St.

9 F. Cingularus. Die umgürtelte Spin. del. Gmel. Murez Nafa. Sp. 23. Martini 4. I. 122. . $113 \mathrm{I} 1132.3 \mathrm{Se}$. 6ig. 7. I St. f. Mast. 4 1. 123. f. 1133.1134. I St.

153510 F. Topete - perficuro. Der perfiche Teppich, Gmel. Murex gibbulus. $\int_{p}$ I 25. Martioi 4. t. 140. f. 1308.9. Knore 5. Tb. 10. fig. 4. $4 \mathrm{St}$.

1536 it F. Anchas. Die Ananas. Gmel. Mu. rex infundibulum. Sp. 108. Liftes Conch. 921. f. 14. Mastioi 4. R. 143. ving. 39. f. A. 2 St.

153711 * F. Striatus. Die geftreifre Spindel. Gmel. Murex verrucofus. Ip. I20. Martini 4. t. 144. F. 1341 . I Si.

1538 II * F. Filarus. Die umwuodene Spia. del. I St.

3540 $12 \mathrm{~F}$. Craticulazus. Die Roft.Spindel. Gmel. Murex craticulatus. Ip. 105. Mar. tini 4. t. 149. f 1382.1383 .5 St.

154113 F Pulcber. Die kleine gegitterte Spindel I St.

$154214 \mathrm{~F}$. Iris. Die Regenhogen S Spindel. Gmel. Murex prismaticus. 'p. 170. Chemn, 10. t, 169. f. 1635, 1636. 1 S. 


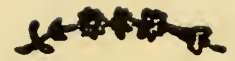

1543|IS F. Purpuratus. Die purpurfarbene

2. Columelle glabra. Mit glatter Acbfe.

I544 16 F. Trompeta. Die Trompete. Gmel. Murex ruba. \{p. IO3. Ein febr grolfes Exemplar. Martini 4. t. I43. f. I333. 7. St.

1545 I7 F. Pyramis. Die Pyramide. Gmel. Murex tuba. fp. 103 var. I St.

$154618 \mathrm{~F}$. Ternatanus. Die Ternaranifche Spindel. Gmel. Murex ternatanus. fp. 107. Marrivi 4. t. 140. f. 1304. 1305. $2 \mathrm{St}$.

1547 Knort 6. 2. I5. f. 4. \& 8.26. f. I. ISt.

\section{Lade 63.}

I548 19 F. Morio. Der gebandete Mohr. Gmel. Murex morio. 'f 62. Matrini 4. t. 139 . f. $1300.17 \mathrm{St}$.

1549 - Mart 4. ז. 139. f. $1301.4 \mathrm{st}$.
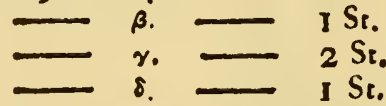

! F. Ateroiops Der ungekerbie Mohr. Gmel Murex morio. 'p. 62. var. I St.

15542 I F. Pugilinus. Die gelbe gezakte Spindel. Gmel. Murex velpertilio Ip. 100. Martini 4. t. 142. E. 1323. 1324. 5 St.

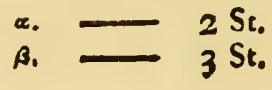


I557/22 F. Carnerius. Die doppele gezakte Spindel. Gmel. Murex refper rilio. Sp. 100 Chemn. 10. t. 164. I I 566. 67. 2 St.

155823 ! F. Deplastatus. Die ausgedrechfelte Spiadel, braun von Farbe obue Binden. Ginel. Murex morto. Sp. 62. var. 2 St.

155924 F. Meroccanus. Die Matocanifche Spindel. Gmel. Murex marocceofis. Sp. J32. Chemo. 9. t. 105. f. 896. 7 St.

Syrinx. Die Sprüze.

1560 I S. Aruana. Das Aruanfche Horn.

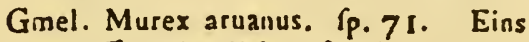
von aufferordeotlicher Gröte, das an. dre kleiner, ajer von vorzüglicher Schönheit. Chemo. 4. Pag. 143. vigu. 39. f. D. Rumpf t. 28. f. A. 2 St.

2 S. Longiffima. Die fehr lange Sprüize. Ginel. Murex longiflimus. 'P. I I 6 . Martini 4.t 145. f. 1344. 2 St.

15623 S. Produal. Die ausgereckte Sptsize. Ginel. Mutex candidus. fp. 113. Martini 4. t. 144. f. 1339.2 Se.

$15634 \mathrm{~S}$. Tabaccaria. Die Tobakspfeife. Gmel. Murex undatus. Ip. I1 5. Mas. tivi 4. t, I45. F. 1343 . I St.

$15645 \mathrm{~S}$. Turnaze. Diegedrechfelte Sprüıze. Gmel. Murex colus. Ip. 6r. Martini 4. r. 144 . E. 1342 . 5 St.

15666 S. Maxima. Die grofle Sprüzze. Gmel. Murex longiflina. Í. I16. var. mic febr fcharfen Reifen, und nicht geknobbelt. I St. 
1567| 7 S. Maculara Die gefleckre Sprürze. Gmel. Murex anfatus Ip. 114. Mas. tini 4. t. J44. f. 1340 . 5 St.

J569 8 S. Nicobaricu. Die Nicobarifche Sprüıze. Gmel. Murcx rolus. ip. 6r. Chemn. 10. 1 160. f. 1523. $\%$. 2 St.

9 S. Mormorata. Die bunte Sprüize. Gmel. Musex colus. fp. 6I. var. Chemn, 10. t. 160. f. 1523. Ist.

1571 $10 \mathrm{~S}$. Viminalis. Die lineitre Sprütze. Gmel. Murex colus. '́p. 6I. var. I St.

3572 II S. Petioleto. Die kleine Sprütze.

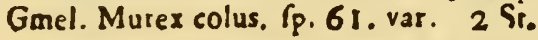

157312 S. Annulete. Die geringelte Sprürze. Gmel. Murex polygoous. Ip. I09. var. Martini 4. 2. 141.E. 1316. ISe.

1574 I 3 S. Clazbrasa. Die gegitrerte Sprürze, Gmel. Murex polygonus. 'P. I09. Mar. tini 4 . t. 141 . f. 1316.4 St.

157514 S. Buccinoidea. Die aufgeblafene Spr. Gmel. Murex vertucofus. fip. 120. Mat. tioi 4. t. 144. f. 1341 I. $^{\circ} 2$ St.

1576 15 S. Parvulus. Die warzige Sprüize. Gmel, Murex vertucolus. 'P. 1 20. Mase tini 4. t. 144. f. 13412 St.

1577 I 6 S. Venofa. Die adrichte Sprūıze. Gmel. Murex verrucofus. \{p. 120. I S

157817 S. Elongcta. Die ausgereckre Sprözze. Gmel. Murex colus. Ip. 61. var. I St.

I579IS S. Ficoidea. Die abgefetzte Sprütze. 2 St.

J580 19 S. Foffa. Die ausgegrabene Sprürze. Gmel. Murex folfilis. 'P. II2. Mare tint 4. t. 141 . . J321. 1322. I S. 


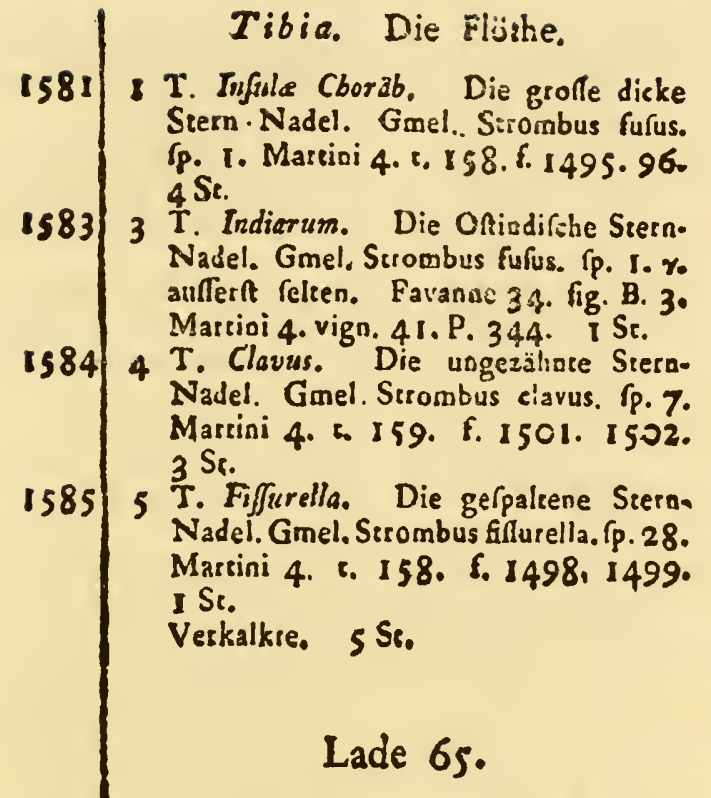

Turris. Der Thurm.

I T. Babylonica. Der Babilonifche Thurm. Gmel. Murex babylonus. Ip. 52. Martini 4. c. 143. E. I33\%. 1332. 7 St.

2 T. Nobilis. Der geringelte Thurm. Gmel. Murex babyloous, fp. 52 . var. 2 St.

15893 T. Pulcbra. Des fehöne Thurm. Gmel, Murex babylonus. fp. 52, var. I St.

4 T. Imperfecti. Der unvollikommene Thurm. Gmel. Murex babylonus. If. 52. yar. $2 \mathrm{Sr}$

$159 \mathrm{I} 5$ :T. Excelfa. Des bole Thurm, 3 St, 
1592 6 T. Favana. Der durchfiehtige Thurm. Gmel. Murex javanus. f́. $533^{\circ}$ Matr. 4. t. 143. E. 1337.1338 .4 St.

15937 T. Texee. Der blasgelbe Thurm. Gmel Murex babylonus. fp. 52. ?. Chemn. IO. t. 162. f. 1550. I551. 2 St.

1594 8 T. Indica. Der Indianilche Thurm. Gmel. Murex babylonus. Ip. 52.8. Martioi 4. T. 345 . f. 1345 . 1346. 4 S. Gorbica. Der Gothilche Turm. 15959 T. Gorbica. Der Gothilche Turm. $159610 \mathrm{~T}$. Alere. Det gelügelte Thurm.

1597 II T. Ruftice. Der sothe Thurm. Gmel. Murex babylonns. 'P. 52. I St

3598 I 2 T. Pyramidalis. Der Pyramiden-Thurm. Gmel. Murex babylonus. Ip. 52. 2 St.

I 59913 T. Vitrea. Der glaferne Thurm. Gmel. Murex babylonus. ip. $52.3 \mathrm{St}$.

1600 I 4 T. Regia. Der Königs-Thurm. Gmel. Murex babylonus. jp. 52. $\beta$. Mar. tint 4. P 1 43. ving. 39. Lt. C. 3 St.

I601 I4 * T. Albida. Der weite Thurm. Gmel. Strombus lividus. Sp. 49.3 St.

$160214^{* *}$ T. Fofjilis. Der ausgegrabene Thurm. Gmel. Strombus lividus. Ip. 49. $\mathrm{Sr}$.

$160314^{* * *}$ T. St. Stepbari. Der Stephans. Thurm. Gmel. Strombus lividus. Sp. 49. Chemn. 9. t. 136 f. 1269.70. I St, 1604 I 5 T. Vetufia. Der alee Thurm. Gmel. Srombus lividus. Sp. 49. I St.

1605 I 6 T. Tormatum Der gedrechfelre Thurm. Gmel. Murex balylonus, Sp. 52. F3. vane Tb. 33. Ig. C. 6. $4 \mathrm{St}$. 


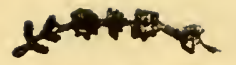

1606/17 T. St Matritii. Det S:. Afotitz Thutm. Gmel. Murex babyloans. Sp. 52. $\%$. Martini 4. P. 143 vign. 39. fig. B. Argeav, zoomorph. 6. 4. E. 6. Sebr. felten, $2 \mathrm{Sr}$.

$160718 \mathrm{~T}$. Operofa. Det künfliche Thurm. Gmel. Strombus vittatus. Sp. 25. $\gamma$. Favanne e. 20. F. A. \&. Chemn, 10. C. 155 . f. 148 1. 82 . 2 St.

160818 * T. Clasbrass. Der gegirterte Thurm. Gmel. Strombus vittacus. Tp. 25. Var. 1 St.

\section{Tritonium. Das See-Horn.}

1609

I T Trizonis. Das Tritons. Horn. Gmel. Murex tritonis. Ip. 89. Mart. 4. 8. 134. f. 1272.1282 .1283$. Kaorr Verg. 2. c. 16.f. 2. 3. \& 5.t. 5. f. I. 9 S. T. B. das gelbe. Mari. 4. 8. 134 . f. 1279. I St.

16122 T. Ncpruni. Das Waffer -Horn. $4 \mathrm{Sr}$. $16133 \mathrm{~T}$. Opis. Das verkürzte Trizons.Horn. Gmel Murex tritonis. (p. 89. $\beta$. Martini 4. t. 136. f. 1284. 1285.2 St.

$16144 \mathrm{~T}$ Filiforme. Das kleire fein granulirse Tritons-Horn. I St.

16155 T. Minusum. Das kleias Tritonstorn. 2 Se.

16166 T. Buccinulum. Die kleine Trompere. Gmel. Buccinum igneum fp. 107. Martini 4. \&. 127 f. 1217 . I St.

1617

7 T. Accinctum Das gefederte TriconsHora. Gmel. Buccinum plumatura. rp. 108. Martini 4. t. 127. .. 1218. 1219. 3 St.

T. 127. f. 1220. 35r. Mastini 4. 
1619| \& T. Mneularum. Das gefleckte TritonsHora. Gmel. Murex maculofus. โp. 79. Martini 4. c. 132. f. 1257. 1258. 18 St.

9 T. Candifarum. Das kandirte Tritons. Horn. Gmel. Murex condicus. fp. 174. Chemn, 10. to I62. f. I 544. 1545. febr felten, I St.

1621 \% Die Frofch-Schnecke. 1 St.

$162210 \mathrm{~T}$. Olearium. Del Oelkrug. Gmel. Murex oleariun. fp. 27. Martini 4. t. 130. E. 1243. Knorr Verg 3. t. 9. f. 5 . ISt.

3623 St.

$1624-B_{0}-$ Mart 4 t.131.

1. 1250 . I St.

1625 II T. Olfactoriolum. Die Riecbbüchfe. Cinel. Murex olearium. ${ }_{p} .27 .2 S_{0}$.

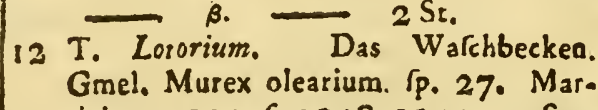
tiai 4. t. 130. f. 1248.1249. 4 St.

162913 T. Labrum. Die Wanae. Gmel. Ma. sex oleariuin. $\int_{p}: 27.2 \mathrm{St}$.

163014 T. Nicobaricum. Das Nicobarifthe Tritons. Horn. Gmel. Murex olearium. fp. 27. Martini 4.t. 132.f 1246.47 . $7 \mathrm{St}$.

$163115 \mathrm{~T}$. Limbatum. Das gefäumte Truronsa Horn. Gmel. Murex olearium. \{p. 27. Martini 3. 6. I32. 6. I259. I Ss.

\section{Lade 66.}

$163216 \mathrm{~T}$. Heparterm. Die gelbe PaternoAer. Sebnecke Gmel. Murex Rubecula. Sp. 35. Martini 4. t. 132. E. 1263. $1264.1 \mathrm{St}$. 
$1633 / 17$ T. Flaveole. Die duakelbraune Pacernofter-Schoecke. Grael. Murex Rubecula. fo 35. Matt. 4 ?. 132.6.1261. 1262.3 St.

$163418 \mathrm{~T}$. Rubecula. Die orangefarbene Pa. rernofter - Schnecke. Gmel. Murex Rubecula. (P. 35. Martini 4.t 132. E. $1265.1266 .3 \mathrm{St}$.

1635 a. 2 St

1636 19 T. Natasor. Der Schwimmet Ginel. Mures gyrinus. 'p. 24. Martuni 4. 1. 128. t. 1229.1230 .4 St.

163720 T. Gyrinus. Die kleine Rrüte. Gmel. Murex gyrinus. 'P. 24. Martiai 4. 128. E. 1233. 1234. 8 St.

1638 - St.

163921 T. Zabick. Die geleckse Kivic Gmel. Murex gyrinus. ip. 24. Mattioi 4. 1. 127. f. 1224. 1225 . 6St.

1640 a. 1 St.

164122 T. Granulare. Das gekórnte TritonsHorn. Gmel. Murex gyrinus. Cp. 240 Martini 4. C 127. E. 1226. 1227. $4 \mathrm{Se}$,

164223 T. Scrobiculator Das geflamme Ti . rons. Horn. Gmel. Murex ferobiculacor. Ip 36. Martini 10.8. 163. f. I556. $1557.2 \mathrm{Se}$.

164324 T. Tuberofum. Das gebukkelie Tri. tons - Horn Gmel. Murex Lampas $x$ is. bet2. Sp. 26. \%. Martini 4. '. 128. f. 1236.1237 . I St.

164425 T. Argo Buccinum. Der gebandete Aigus. Gmel. Murex argus 'p. 78. rehr felcen. Martini 1223. Knorr 5. c. $3.13 .7 \mathrm{Si}$. 
164526 I. Rubera. Der Garten. Frofch. Ginel. Murex Lampas. Í. 26. \%. Rubera. 5 Si.

1646 a. Dünfchaligre. Martisi 4. t. I28. E. 1236.1237. 2 St.

1647/27 T. Bufo. Die grofe Kröre. Gmel. Murex Lampas. IP. 26. B. Bubo. Mastini 4. t. 129 f. 1238.2 Si.

$164828 \mathrm{~T}$. Helcor. Der Hector. Gmel. Mi. rex Intorium If. 30. $\beta$. I St.

164929 T. Simpulum. Gmel. Murex locorium. Pp. 30. B. Martini 4. t. 31.f. 1254 7 S.

1650 a. Ginel. Murex lotorium. Ip. 30. B. Mlartini 4. R. 13 I. f. 1252.1253 . I Se.

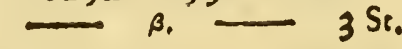

\section{Lade 67.}

$165230 \mathrm{~T}$. Reticulans. Das gegitterte Tricons. Horn. Gmel. Murex retrculans. 'p. 37. Martini 4. t. 128. f. 1228 . 6 St.

Burfa. Die Talche.

1653 I B. Rana. Der Frolch. Gmel. Murex sana. Ip. 23. Martini 4.t 133.f. 1276 . $6 \mathrm{Sr}$.

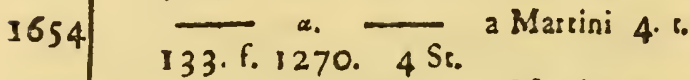

$1655 \quad-\frac{}{32}$ a Marriui $4 . t$.

2 B. Gibbofa Die bauchigte Talcbe. Ginel. Murex rana. If $230^{\circ}$ a Mart. 4. t. 133. f. $1273.2 \mathrm{St}$.

16573 B Mammara. Die watire Kı̈̈re. Gmel. Murex bufonins (p. 32. Favanne Tb. 32. fig. B. 1. Mart 4.t. 129. f. 1240. 4 I. 2 St. 


\section{setrots.}

161413 B. Monisere. Die eigefefarste TaFhe Gmel Murex bufonius. Ip. 23. I St.

16 Is 4 B. Bufonis. Die ftachlige Rröte. Gmel. Murex raoa. ip. 23. $\beta$. Mattini 4 c. 133. f. $1274.12 \% 5.7$ St.

Cymatium. Die Kebl-Leifte.

I C. Femorale. Das dreyeckige Kink Hora. Gaxel. Murex femorale. '́p. 28. Martiai 3. t. 111. f. 1039. Koors Verg. 4.6. 16. . I. 7 St.

2. C. Rbinoceros. Die Hautfchnecke. Gmel, Murex pyrum. Sp. 33. Knoss 6 t. 26. f. 2. 4 Si.

\section{Lade 68.}

1621

1622

1623

1624

1625

1626

1627

1628

3 C. Pyrum. Die getrockaete Birne. Gmel. Murex pyram. Sp. 33. Marr. 3. t. 112 . f. $1040-1044$. 4 St,

4 C. Maculatunt. Die gefleckte Butne $2 \mathrm{St}$.

5 C. Clavasum. Die gegisterte Birne. Gmel. Murex pyrum ip. 33. I St.

6 C. Canalicularum Die brãundiche Birae. Gmel Murex pyrum. Ip. 33. 2 St.

7 C. Caudatum. Die weiffe keilförtmige Biroe. Gmel. Murex pyrum. $\Upsilon_{p} 33$. $2 \mathrm{St}$.

8 C. Flexuofum. Die gebogene Birne. Gmel. Murex pysum. $\int_{p} .33$. 2 St. 
9 C. Muricotumt. Die dosoigte Birne. Gmel. Murex pyrum. Ip. 33. I St.

Cabeftana. Die Winde.

I C. Cymatium. Das knotigte Weinfafs. Gmel. Musex cutaceus. $\int_{p}$. 29. Mar. tiai-3. ז. 118. F. 1085: 1086. 1 St.

2 C. Doliata. Das geripte Weinfals. Gmel. Murex curaceus. 'p. 29. Die Hautfehnecke. Martint 3. to 118. f. $1087.88 .8 \mathrm{St}$ 。

3 C. Coftata. Das dünfchälige geripte Weiufafs. Gmel. Murex cutaceus, fp. 29, Martioi 3. t. 118 . f. 1087.1088. 2 St.

4 C. Doliolum. Das Fälsehen. Gmel. Mu. rex deculfaras. Ip. 9 Marcini 3. t. 110. f. $1027.7 \mathrm{Si}$.

5 C. Helclarum Kabeftan, oder die Schifswinde. Gmel. Buccinum feala. 1p. 6r. Martini 3. c. 1 18. f. 1089. Knors Verg 3. t. 7. f. $2.2 \mathrm{St}$ -. Schrötets Einleit. in d. Conch. 1. P. 360. t. 2. 6.8. 2 St.

6 C. Difformiss. ${ }_{\text {Die ungeftaliene Wiode }}$ I Si,

Nucella. Die Nufs.

1641 N. Rericulata. Die gegitterte Nufs. Martini 3. 1. 121. f. 1107. 1108. 3 St. 
1642 2 N. Moscbatellina. Die Museat. Nufs. Gnel. Burcinum laeve, Ip.72. Mat. tini 4 t. 124. f 1150. 3 St.

16433 N. Macira. Die groffe Muskat-Nufs. 2 St.

$4 \mathrm{~N}$. Lapillus. Die Aeinigre Nufs. Gmel. Huccisum tulticum. rp. 65: Martini 3. f. 120. f. 1104. 1105. $6 \mathrm{St}$.

$16455 \mathrm{~N}$. Tbeobroma. Die Kakao - Nuls, Gmel, Buccinum filofum, fp, 67. Marsioi 3.t. $121, f .1113 .1114$. ISt.

Lagena. Die Flafche.

1646 I L. Undofa. Die gewellie Frafche. Gmel. Bucciaum undofum. 'p. 84 . Martini.4. I 122. f. 1126. 1127. 7 St.

16472 L. Cresulata. Die wulntige Flafche, Gmel. Buccinum undofurm. lp. 84. Martuni II 45 . I 146. ISt.

$16483 \mathrm{~L}$. Violaces. Die blauliche Flafelie. I Se.

16494 L. Elegantiffina; Die orangefarbene Flafche. $2 \mathrm{Sc}$.

1650

5 L Varia. Die bunte Flarche. I Se.

6 L. Nodulofa. Die knotige Flafche. I St.

7 L. Pterygiata. Die ge日ügelte Flalche. 2 St.

8 L. Nigelle. Die rchakigre Flafche. Gmel. Buccinum ocellatum. [p. 73 . Martiui 4. i. 124. f. 1160.1161. I St; 
- Nadfa. Die Fifch-Reule.

I N. Pifta. Die gemahlce Fifchreufe. Gmel. Buccioun corobatum. 's. 68. Maitini 3. t. I2I. F. II I5. I I I6. $5 \mathrm{St}$.

N. Cafia. Die braune Fifchreufe. I St.

16573 N. Flaveola. Die lichtoranne Fifch. reufe. I St.

4 N. Striata. Die geftreifte Fifebreufe. I St.

16595 N. Duplicata. Die weifsgelbe Fifch. reure. I St.

6 N. Ligata. Die gebandere Fifchreufe. Gmel. Buccinum flofum. Ip. 67. Martini Tom. 3. t. 121. f. II13. I114. 1 St.

7 N. Rudis. Die plumpe Fifehreufe. Gmel. Buccinuin lapillus. (p. 53. Mattini 3. t. 121. f. III I. III2. 4 St. 8 N. Harpa. Die Harfe. I St.

9 N. Argiolus. Die augichte Fifchreufo. 1 S.

Cantharus. Die Kanne.

1664 I C. Globularis. Die Kugelformige Kanne. Gmel. Buccioum rranquebaricum. Ip. 86. I St.

16652 C. Tubcrofus. Die knolligise Kanne. Gmel Buocinum undofum. 84. 84. Martini I 146.1147 .5 St.

16663 C. Scalaris, Die gewuodene Kanne. 3 Sr. 16674 C. Nodofus, Die knosige Kanne. 
J668| \& C. Erofus. Die bletrigizinane Gmel Murtex hipporaftazime. TP. 84, Mart.3 ז. 100. f. 954.55 .

5 C. Hippocaftanumar. Die wilde Câfravie. Gomel. Minrex kigpacaftanum. f. 48 . 6 St. 1670 f. 951.4 St. Mastini 3.t. 100.

16715 "* C. Tribuloides. Die gecoonte Kanne. ISe.

16726 C. Triplicatus. Die gefultete Kanne. Gmel. Buccinum pyruzoniss. P $_{p}$ 7r. Martini 3. to 109. f. 1017,4 St.

Diftorflo. Das Obr-Horn.

1. Caudate Gefelowanzie.

I D. Anas. Das Ohrhorn. Gmel. Murex anus. \{f. 38. Martivi 2. t. 41 I. ., 403. 404. Knorr 3. t. 3. f. 5.5 St.

2 D. Reticulesa Das gelbe Obrhorn. Gmel. Murex anus. Íp 38. var, Mart.2. t. 4 1. f. 405.405 . 5 St.

3 D. Cletrata. Das weife Ohr - Horn. Gmel. Murex anus. fg. 38. variesas. St.

4 D. Muricina. Das Rachliehic Chr. horn. Gmel, Murex pyrum. fp. 33.8. Martini 3. 8. I12. f. 1050. 5\%. 4 Sis

2. Truacazce. Abgefutze.

1677 S D. Arcularia: Das kofferartige Ohr. Gmel. Buccinum arcularia. Sp. 42. Marcioi 2. ab. 41. f. 409.410 . $6 \mathrm{Sh}$ 
Lade.69.

16786 D Plieata. Das gefaliere Ohrborn. Gmel Buccinam areolaria. If. 42. Martin 2 1.41. 6. 411.412 .4 St.

16797 D. Dealbazs. Das gelbliche Ohrhorn. Gmel. Buccinum areularia. $I_{p}, 42$. var. 3 St.

$16807^{*}$ ! D. Subalats. Das geflügelte Ohs. born. Gmel Buccin. arcularıa. Sp. 42. var. I St.

8 D Communzs. Das duakelbraune Ohto born. Gmel Burcinum artularia. 'P 42. var. Martioi 4. t. 124. f. 1169. 4 St.

Xancus. Das Sianko-Horn.

I X Pyrum. Die Birne Salbhoro. Gmel. Voluta pyrum. '́p. 102. Martini 3. t. 95. f. 916. Knorr 6. t. 29. f. I. 2 S.

2 X. Punktatus. Die bunte Birne, Gmel. Volura pyrum. ip. 102. $2 \mathrm{Sr}$.

3687 - Martiol 3. i 95 f. 918. Knorr 6. t. 27. f. 2. $3 \mathrm{St}$

3 !! X Perverfus. Das links Sianko. horn, aufferit felten Chema. 9. t. 104. f. 884.885. \%. I St.

\section{Pyrene. Der Judenftein.}

1689 I P. Rbombiferum. Der gefleckrc JudeoRein. Matrin 2. 1. 4.4. f. 465 Det goldgelbe weiffe Olivenkers. ISt. 

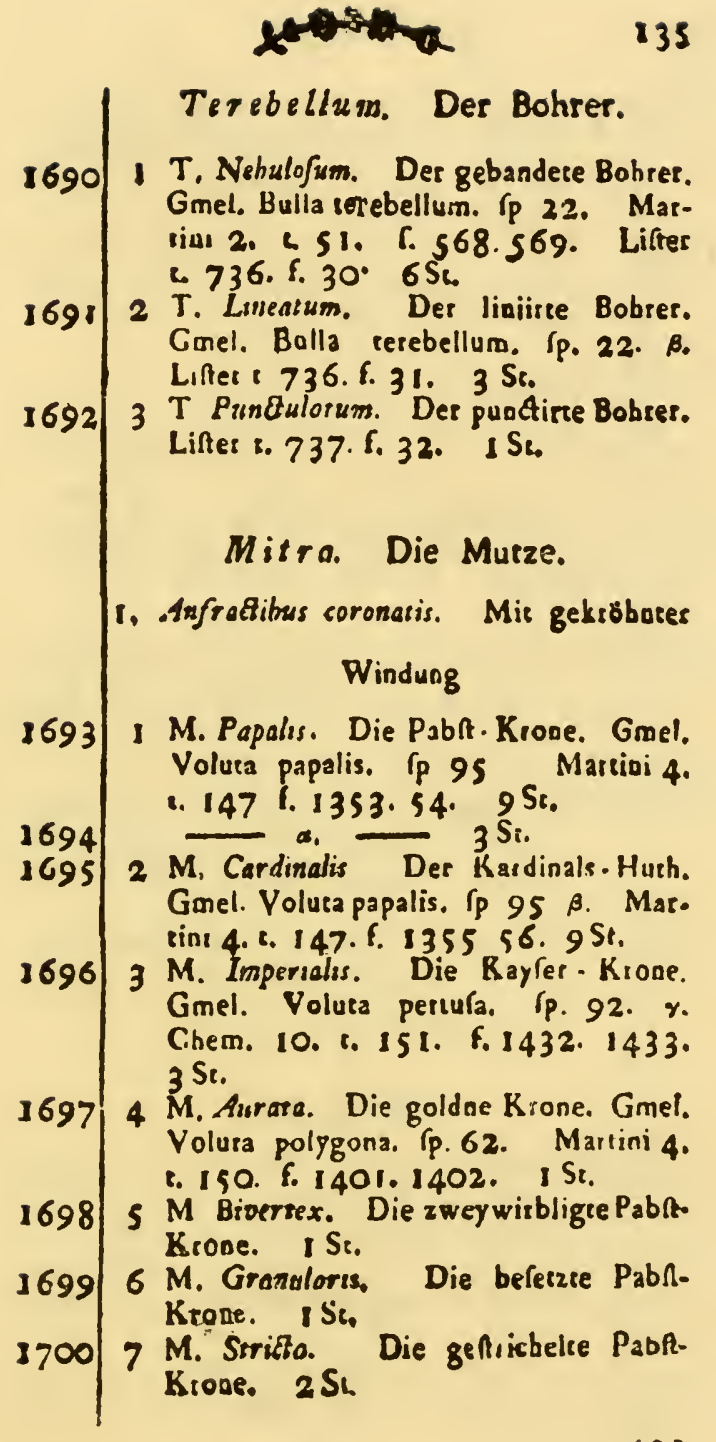


\section{$20+6$}

701 $\&$ M. Bernbardina. Die Bernhardiner Pabat-Krone. Gmel. Volura parriarcha. lis. Ip. 138. Chem. 10 I 150. f. 14272 St.

8 * M. Perlata. Die geperlte Pabrt. Krone. Gmel. Voluta pertula. Ip. 83. var. Ieltea. 2 St.

2. Anfractibus contiguis. Mit as eioander Rehender Windung.

9 M. Episcopalis. Die Bif́cbofsmürze. Gmel. Volura epifcopalis. \{p. 94. Mar. trini 4. r. 147. F. 1360.1360 .2$. $9 \mathrm{St}$.

$10 \mathrm{M} \cdot$ Carmelita. Die Carmelitermürze. Gmel. Voluta epifcopalis. Spec. 94. $2 \mathrm{Si}$,

II M. Monacbialis. De: Mönchs . Hurb. Gmel. Volutz cardinalis. Ip. 93. Martini 4. tab. 147. f. 1358 . 1359. 5 St.

170812 M. Pertufa. Die durchfochene Pabft. Krone. Gmel. Voluta pertula. fp. 83. Kammerer 9. fig. 8. I St,

\section{Lade 70.}

I 3 M. Eremizarum. Der Einfiedler-Huth, Gmel. Voluta pertura. fp. 93. Mar. tini 4. t. 147. f. I36 I. 3 St.

$171014 \mathrm{M}$. Capucina. Die Capucinermürze. Gmel. Voluta pertufa. Ip. 93. varietas I St.

17II $\longrightarrow$ a. ISt.

1712 I5 M. Lutes. Die gelbe Birchofsmürze. 2. St. 
I713!16 M. Inops. Die weilsgefpraakelte Bifchofsmürze. 2 St.

$171417 \mathrm{M}$. Difrolor. Die weifsgebandete Bifchofsmŭtze. Groel. Voluta fcurulata. Sp. 131. Cheran. 10. c. 151. f. 1428. $1429.5 \mathrm{Sr}$.

$371518 \mathrm{M}$. Caftanea. Die caftanienbraune Bifchofsmüzze. Gmelin Voluta nigra. 5p. 132. Chemn, 10. 2. 151, f. 1430. 1431 . I St.

17 I6 $19 \mathrm{M}$. Vittata. Die tuntfchackigte BiSchofsmürze. $3 \mathrm{St}$

$171720 \mathrm{M}$. Venofa. Die adrigte Bifchofsmütze. Gmel. Volura paupetcula. Ip. 37. Martini $4^{\circ}$ tab. 149. f. 1386.1387. 3 Se.

1718 2 I M. Nivea. Die febreeweife Bifchofsmŭıze. I Se.

171922 M. Minuta. Die kleine Bifchofsmütze.

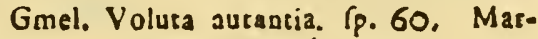
tini 4. tab. 150. f. 1393. 1394. I St.

172023 M. Crenulata. Die ausgezackre Ri. fcliofsmütze. Gmel. Voluia crenulata, Ip. 130. Chemn. 10 1. 150. f. 1413. 1414. Selten. I St.

3. Anfractibus diftunezs. Mit voa einander abgefonderter Windung.

172124 M. Elata. Die fengegitcerte Bifchofs. mürze Gmel. Voluts acuminaı́ 'p. 63. Marc, 4. tab. 150 . f. 1403.1404. I Se.

$172225 \mathrm{M}$. Cinnamomed. Die grobgegitretre Bifchofsmuirze $3 \mathrm{St}$.

172326 M. Elegantifima. Die niediiche Bifchors. müize. I St. 
$1724 / 27$ M. Pulcberrima. Die fchỏne Bilchofs mìzze. 3 St.

172628 M. Praftantifima. Die überaus £chöne Bilchotsmütze. Gmel. Voluta lcabricula. SP. 48. Matt. 4. ז. J49. E. 1388.89. 4 Si.

172729 M. Flammeola. Die gefammie Bilchos. mürze. ISt.

172830 M. Sanguifuga. Die mit Blut gezeich. Dete Bilchofsmŭrze. Gmel. Volure fan. guifuga. Sp. 50. Martini 4. t. 148. f. 1373 . S St.

31 M. Sanguinea. Die blutrothe Bifchofs. müsze. I St.

$173132 \mathrm{M}$. Cnncellata. Die gewütfelte Bifehfse muize. Gmel. Voluta granofa. 'p. 136. Chemn. 10. t. I5I. I. I442. 1443. I Se.

$173233 \mathrm{M}$. Caleta. Die gefteifie Bifcbofs. Müzze. $r$ St.

$173334 \mathrm{M}$. Nobilis. Die orange Bifebofse Mürie. I St.

173435 M. Margaritaced. Die gepetite Bi. fchols-Muitze, ISt.

Vexillum. Die Fahne.

1735 V. Foffile Die weifle Flagge. I Si,

$17362 \mathrm{~V}$. Plicatum. Die gefaltete Flagge. Gmel Voluca plicaria Sp. 55. Mars.4. r. $148 . \mathrm{f}_{1362.63} 4 \mathrm{St}$.

17373 V. Lividum Die bleyfarbige Flagge. Gmel Voluta plicaria Sp. 55. $6 \mathrm{St}$.

$17384 \mathrm{~V}$. Variegatuin Die veranderlicho Flagge. Ginel. Voluta variegate. Sp. 89. Knotr 5. t. 18. 6.6. 

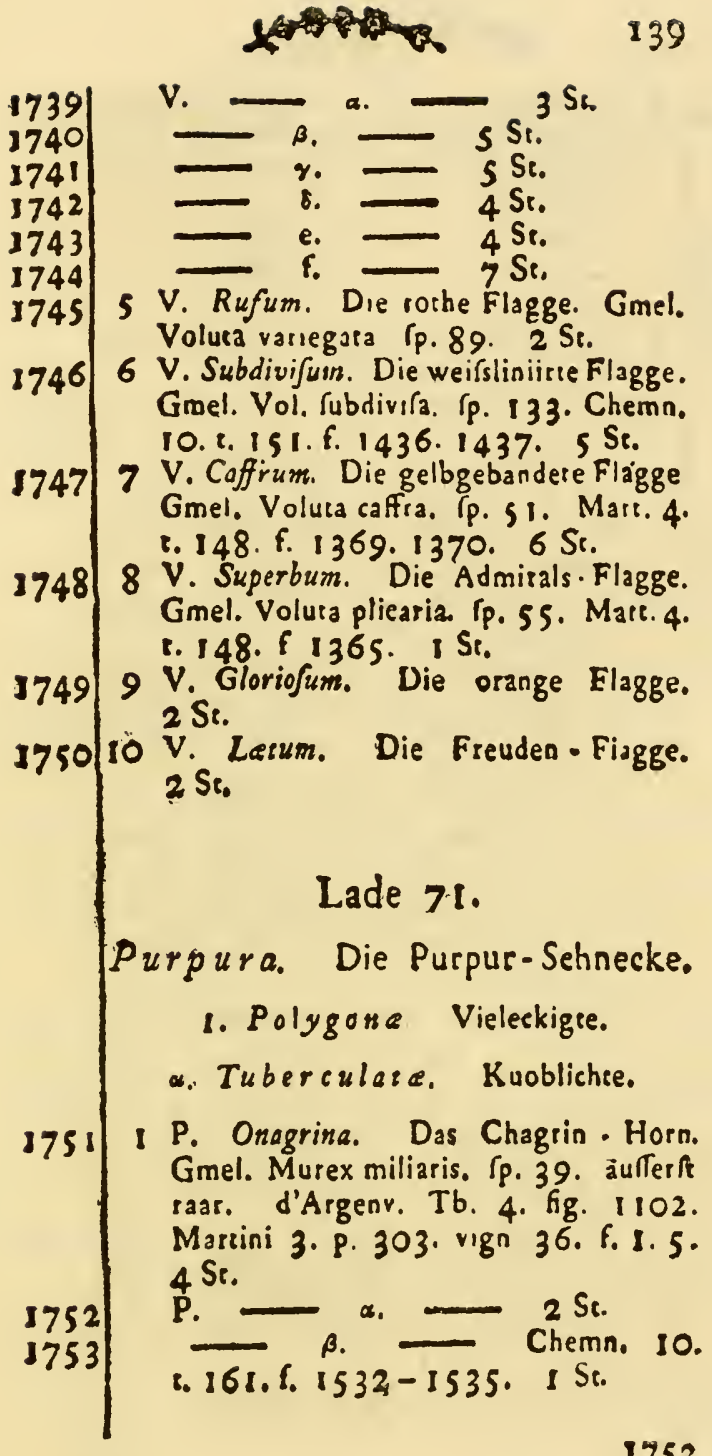
2 P Trunculu, Der braungezakie Hoch. Fcbwanz Gmel. Murex erunculus. ' $p .5$. Marrini 3. t. 109. f. 1018-1020. $14 \mathrm{St}$.

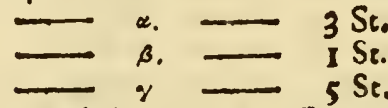

3 P Tribulus Die Waffer $\cdot$ Nufs. Gmel, Murex stunculus. fp. 5.5 St.

B. Crispę e. Gekräufelıe.

4 P. Buccinulum Die ãcbee Bords. Treppe rebr felcen. I St.

5 P. Lomellare. Die Bords- Treppe, 2 re Sorte. I St.

6 P. Senegalla: Die Senegallche Purpur. Schnecxe. "Ginel. Murex renegalenfis. Sp. 40. Martini 3. t. I10 f. 1027. 10285 St.

7 P. Coftara. Die geribte Putpur-Schn, Ginel. Murex coftacus. 'p. 86 . $6 \mathrm{St}$.

8 P. Congener. Die weilsgelbe Purpur. Schnecke. Gmel. Murex deculasus Sp.7. Maruni 3. ¿. 110. f. 1026 Koorr 4. t. 23 .f. 3. I St.

9 P. Crispara. Die kraule Purpur - Schn. 9 St.

176510 P. Acura. Die rcharfe Purpur. Schn. I St.

1766 is P. Rubicunda Die rothliche Puspuro Schnecke. Gmel. Murex trunculus. IP. 5 . $3 \mathrm{Se}$.

176752 P. Rofarium. Die sofearothe Purpuse Schuecke Gmel. Murexirunculus. IP. 5 . ३. Chemn. 10.1 161. . 1528. I529. $3 \mathrm{Sr}$. 
$1769 \mid 13$ P. Virginea. Das jungfern. Horn. Gmel. Murex samofius. $r_{p}$. 13. S. Mas. tini 3. t. 110. f. 10307 St.

1770 f. 1029. 3 St.

\section{Lade 72.}

177114 P. Duplex. Die Purpur. Schnecke mit blussother Mindang Gmel. Murex \{axa. silis. Ip 15. B. Mastini 3. to 108. f. 1013 . 1014. IS.

7 Fimbriato frordofa. Mit Lanb bewachlen.

1772 I5 P. Lastuca. Der Kopfralas, Gmel. Murex Taxatilis. 'P. I5. Martini 3. i. 107. f. 1005-1008. 3 St.

1773 a. Mart. 3. 8. 107. f. $10 \mathrm{rr} \cdot 1012.4 \mathrm{St}$.

177416 P. Scorpro. Des Scorpion Gmel. Mu. rex fcorpio Íp 34. Martini 3. 8. 106. f. 998 - 1003. Knors 2. C. 11 . f. $4.5 .2 \mathrm{St}$.

177517 P. Elongara. Die ausgeftreckse Purpur. Sclinecke. I St.

377618 P. Tulerofa Die mir fchwarzea Strab. len beferzte Purpur - Schuecke. Gmel. Murex melanamarhos. ' P. $_{\text {. }}$ 9. Mas. tini 3.t 108 f. 1015.3 St.

177818 !! P. Marsinesana. Die gefenferte Purpur Schnecke. Gmel. Murex colus. ip. 61. 8. Chemn. 10. s. 161. \& 15361537.1 St.

1779 I9 P. Spinule. Die gedornce Purput. Schoerke. I St. 
1780 I128. $* *$. $\mathrm{rSt}$ Four diurerg

2. Trigona. Dreyeckigte.

1. Cuspideza. Zugelpitzte.

1781 $20 \mathrm{P}$ Carnu Cervi. Das Hirfebhora. Gmel. Murex ramolus. fp. 13. Ein Exemplar fehr grofs. Martini 3. t. 102. f. $987.988 . \quad 3 \mathrm{St}$.

178221 P. Cormu Dama. Das Damm Hirfeh. Hora. eod, $6 \mathrm{St}$.

B. Frondosa. Aeftige.

178322 P. Prunnea. Die braune Purpur-Scho. eod. 6 St.

$1784={ }^{\alpha}$ Se.

178522 * P. Quadricornis. Die vierhornigre Purput -Schnecke. eod. 2 St.

178623 P. Ramofa. Die lineirte Purpur-Sehn. eod. 7 St.

178824 P. Gemella. Die lichtbraune Purpuse Schrecke. $4 \mathrm{St}$.

\section{Lade 73.}

178925 P. Lactea. Die milchweife Purpur. Schnecke. 4 St.

179026 P. Uncinata. Die mit Hacken befetzte Purpur - Schnecke. 2 St.

179127 P. Incarnata. Die röthliche PurpurSchnecke. Martıni 3. t. 102. f. 980. 981. II St.

179228 P. Carneola. Der Rofenftock. eod. Martini 3.t. 102. f. 995.996 .3 St. 


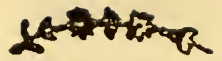
I793/29 P Brandaris. Das Brandhorn. Gmel. Murex brandaris. Sp. 4. Knorr Verg. 2. t. 18. f. 1. 2. \& 22. f. 4. 5. $12 \mathrm{Sr}$.
179530 P. Fuliginosa. Die Rulsfarbene Purpus. Schoecke. Gimel. Murex brandaris. Ip. 4. 8 Se.

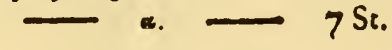

r. Opsufa. Abgellumpfre.

31 P. Capucine Die Kapuziner PurpurSchnecke Kerrenhörmer (mır ftumpfen Nathen.) Gmel. Murex samofus. \{p. 13. Four d'Auverg. $1073 . \quad$ \%. Martini 3. t. 105.6 .994 .4 St.

32 P. Variegatia. Die fchäckigte Purpur: Schuecke. Gmel. Murex ramofus. 5p. 13. द. Martini j. t. III.f. 1038. $3 \mathrm{St}$.

33 P. Cancellata. Die gegitrerte Putpute Sclsnecke Gmel, Murex samolus. IP. 13.' 3. Martini 3. t. 11 1. f. 1038. $2 \mathrm{St}$.

33 * P. Defpects. Die Zabnreiche PurpurSchnecke. I St.

180334 P. Truncara. Die abgeftumpfe Pur. pur. Schnecke. Gmel. Murex decufta. tus. Ip 7. Chema. 10. 161. f. $1540.41 .2 \mathrm{St}$.

\section{Lamellata. Blattrige.}

180435 ! P. Foliara. Die blärrige Purpur. Schnecke, Gmel. Murex foliaru?. Sp. 174 felien. Chemu. 10. t. 161. 6. 1538.1539. Martyo unir, Coach. 2. $1.66 .2 \mathrm{St}$. 
1805/36 P. Alata. Die weiffe gefiügelte Put. pur-Schnecke. Grrei. Murex samofus fp. 13. E. Mart. 3. B. III. f. 1036 1037. I St.

180637 P. Crenulara. Die gekerbre Purpur Schuecke. Gmel. Mifurex alper. Ip. 60 Brander Foffilia Hanconienfia. p. 3. f. 77.79. ISt.

180738 P. Fulfformis. Die geipindeltc Purpace Schnecke Gmel. Murex ramolas. fp. 13. 3 St.

180939 P. Draco. Der Drache. Gouel. Murex ruba. fp. 103. Martioi 3. 8. 111. 6. 1033.2 St.

Murex. Die Stachel-Schnecke,

I M. Hauftuiluzm. Der Schoepfenkopf Gmel. Murey bau?el!uno. Tp. 1. Mar. timi 3. t. $115 \cdot$ f. 1066. io St.

$1811-2$ St.

1812. * M. Purpuroides. Des Atachlige Schnepfenkopf. Gmel. Murex bauftellum. Ip. I. var. fehr felten. I St.

1813 I M. Scolapaceus. Der keilförmige Schuepfea.Kopf. Favanue Tb. 38. fig. B. 2. 2 St.

$18142 \mathrm{M}$. Cornutus. Die gezakte Herkules. Keule. Gmel. Murex cornuzus. \{p. 3. Martini 3. t. I 14. f. J057. 22 St.

$18153 M$. Clava Herculis. Die Herkules. Keule. Gmel. Musex brandaris. \{p. 4. Ist.

1810 4 M. Tuberculatus Der weife Schnep. fenkopf. Gmel. Murex braodaris. Sp.4. Martinl 3.t. I14. f. 1058. 2 St 


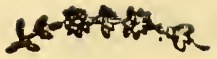
\begin{tabular}{l|} 
Lade 74. \\
3817 \\
5. Trapa. Der einfache Spinneokopf. \\
Gmel. Murex tribulus. Sp. 2. a. Mlar-
\end{tabular} tini 3. tab. I13. f. 1055. 1056. 3. St.
1818 $6 \mathrm{M}$. Tribulus. Der Firsangel. Gmel.

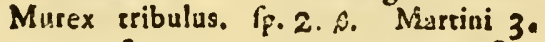 t. I13. f. 1051. $1052-1054.9 \mathrm{St}_{\text {. }}$
18197 M. Forskoeblii. Der forskoehlifeche Spinnenkopf. Gmel. Murex tribulus, Sp. 2. Der feltne gebandete Arabifche. 2 St.

\section{Lade 75.}

18208 ! ! M. Hiftrix. Der doppelte Spinnenkopf. Gmel. Murex tribulus. fp. 2. Fa. vanoe Tab. 38. fig. A. I. \& 2. Knorrs. t. 26. f. I. $2 \mathrm{St}$.

$T$ zisla. Die Kuichenkelle.

I T. Carizata. Der gekehlite Schoepfer.

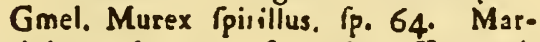
tini 3. tab. I15. f. 1069. Knotr 6. t 24 . f. 3. 3 St.

1822

2 T. Gurturnium. Der Schoepfer. Gmel. Murex pyrum. [p. 33. B. Markini 3. 1. II2. f, 1048. 1049.2 St.

\section{Pile. Der Ball.}

1823 I P. Marretou. Gmel. Helix ampullacea. Sp. 43. B. Chema. 9. t. 128. f. 1136. 3 St.

2 P. Equefrris, Der gebandere Ball. Gmel, Helix ampullacea. \{po 43. 2 St。 
I825 3 P. Periscelis. Der weifle Ball. Gmel. Helix ampullacea. Sp. 43. 8. d'Argenv. c. 17. fig. B. $2 \mathrm{St}$.

4 P. Ampulacea. Das blave Band. Gmel. Helix ampullacea. Ip. 43.थ. Chema. 9. r. 128. f. $1833-35.7$ St.

5 P. Sepulta. Det ausgegrabene Ball ron Coursaignon. Gmel. Helix ampullacea. Sp. 43. 3.St.

6 P. Ignote. Der lchwarze Ball. Gmel. Helix ampullacea. 'p. 43. I St.

\section{Lade 76.}

Cochles. Die halbe Kugel.

I C. Albulo. Die weiffe halbe Kugel. Gmel. Nerira 'padicea, 'p. 8. Chem. 5. t. 188. f. 1896 a. b. 2 St.

2 C. Explanata. Die abgebleichre halbe Kugel. Gmel. Nerita orienralis. Sp. 12. Chemn. 5. t. I88. f. 1904. I St.

3 C. Correa.. Die horufarbige halbe Kugel. Gmel. Neriza Spadicea. Spec. 8. ist.

4 C. Canrens. Der Schmetterling. Gmel. Nerita canrena. 'pec. J. Martini 50 ז. 186. f. 1860. 61. II St.

$$
\text { C. } 1 \mathrm{~S} \text {. }
$$

5 C. Als Papihonis. Der Schmetrerlingsfiigel. Gmel. Nerita canrema. If. 1. s Clemn. 5. 1. 186. 6. 1868-1871. Knorr 1. r. 10. f. 5. 1 St.

$18356 \mathrm{C}$. Claufa. Die ungenabelte balbe Kugel. 2 St.

C. Flemmea. Die Aammigte halbe Kugel. Gmel. Nerita vitrata. Sp. 18. Chemo. 5. t. 188. 6. 1917.2 St. 


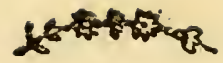

1837 $8 \mathrm{C}$. Fonel. Die buntfchäekigte halbe Kugel. Gonel Nerita canrena. Sp. 1. $\varepsilon$. Chem. 5. T. 187. f: 1876.77. 7 St.

9 C. Stercus Muscartum. Die geftippelte halbe Kugel, Gmel. Nerita canrena. fp. 1. 3. Chemn. 5. t. 187. f. 18781880. Nach det Befchreibung, aber niche die Figur. I St.

183910 C. Ziczac. Die Ziczac halbe Kugel. Gmel. Nerita fulminea. fp. 10. Chern. 5 . c. 187. f. 1881. 3 St.

$184^{\circ}$

1841 It C. Undulata, Die wellenförmige halbe Kugel. Gmel. Nerita canrena. 'P $r$. $x$.Chemn. 5..t. 187. f. 1885.1886. i Se

184212 C. Milleporia. Die punctirte halbe Kuga!. Gmel. Neriza cantena. Sp. I. B. Chem. 5. I 186. f. 1862.63. 2 St.

184313 C. Tigrisa. Der Tieger. Gmel. Nerita canrena. Sp. I " Chemn 5. t. 187. f. 1892.93 .4 St.

184414 C. Once Die Unke. Gmel. Nerirs canrena. Ip 1. $\downarrow$ Chemin. S. t. $18 \%$ f. 1887.88 . I St.

1845 Is C Pavimentum Die wiirfliche halbe Kugel. Gmel. Nerita canrcua. Ip. I. $\downarrow$ Cheina. 5. \&. 187. f. 1889.90. 2 Si.

184616 C. Lineata. Die linēitre halbe Kugel. Cmel. Nerita canrena. Ip. 1. \%. Chem. 5. t. 186. f. 1864.65. 4 St.

184717 ! C. Plicata. Die gefaltere halbe KuGmel. Nerita rugofa. Ip. 14. Chem.5. :. 388. f. 1902.1903 . : St.

184818 C. Sulcata. Die gefurchte halbe Kugel.

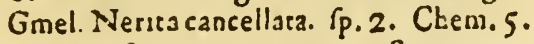
c. 188 . . 1911.1912. IS:。

$$
\text { K. } 2
$$


$1849 / 19$ C. Vitellus. Der Eydotter. Gmel. Nerita vitellus. fp. 4. Chema 5, i. 186. f. $3866.67 .2 \mathrm{St}$.

185020 C. Rufescens. Die braune welfsgebandete halbe Kugel. Gmel, Nerita Tpadi- $^{-}$ cea. T. $_{\mathrm{P}}$. 8. Chemo, 5. . 187 . f. 1872. I873. 2 St.

\section{Ficus. Die Ferge.}

185I I F. Commuris. Die gemeine Feige. Gmel. Bulla ficus. Ip. 14. Kuore Verg. 3.t. 23. f. I. . 9 Si.

2 F. Variegata. Die bunte Feige. Gmel. Bulla ficus. fp. r4. Mattini 3. t. 66. f. 734.735 .7 S.

3 F. Fisit. Die rchāakigte Feige. cod. a. S:。

\section{Lade 77.}

\section{Rapa. Die Rube.}

I ! R. Globoja. Die weifle Rübe, dickfchaligt, mit vertiefter Spirze. Gimel. Bulla rapa. Ip. I5. 2 St.

I R. Rapbanus. Der Retrig. Gmel. Bulla rapa. If. I 5 . I St.

2 R. Striata. Die Radies. Gumel. Bulla rapa. Ip. 15. Martini 3, t. 68. f. 747749. 2 St.

3 R. Pellucida. Die fchneeweifre Radies. Gmel. Bulia rapa. '́p. 15. Martini 3 . I. 68. 6.748. I St. 
$1860 \mid$ \& R. Volema. Die grofe gezackre Rübe. Gmel. Murex sapa. โp. 68. Martini 3. t. 68. f. 750-753. Knort Verg. 5. 2. 21 . f. 2. ISt.

\section{$B u \int y c 0 \pi$.}

I B. Mrrricantm. Die Rachlige Feige. Ginel, Murex carica f́p. 67. Mart. 3. t. 67. f. 744. \& t. 69. f. 756 . 5 St. 2 B. Perverfuns. Die linksgewundeae Feige. Mastini 9.6 106. f. 902. 3. $2 \mathrm{Se}$

f. 900. I. I S:.

Mare. 9 t. I06.

3 B. Dubiumb. Die groffe linksgenua. dene Feige. I St.

18694 B. Cingulatum. Die gegürtelte Feige. 2 St.

5 B. Inverfum. Die gegüıtelce linksgewundene Feige. Ist.

6 B. Cantaliculatumb. Die gekrönte Feige. Gimel. Murex canalicularus, كp. 65. Martini 3: t. 67. f. 742.743. 5 St.

\section{Lade 78.}

\section{Harpa. Die Harfe.}

1872 I H. Mnjor. Die grofle Harfe. Gnel. Buecinum harpa. Sp. 47. Martini 3. c. I19. f. 1090. Koost Verg. I. t. 9. E. 3.\& 2. . . 8. f. 2 . $15 \mathrm{St}_{0}$

$1873 \longrightarrow a_{0} \longrightarrow$ I St. 
187412 H. Cytbara. Die Zitter. Gmel. Bucci. nuin harpa. $\int_{p} 47.18 \mathrm{St}$

3 H. Nabilis. Die edle Harfe. Gmol. Buccinum harpa. Ip. 47. Martini 3. c. I19. f. 1091 . I St.

$4 \mathrm{H}$ Doris. Die rothe Harfe. eod. Martini 3. 2.119. f. 1094 3 St.

$5 \mathrm{H}$. Amouretta Die Halfe mie Ichma. len und breiten Rippen. eod. Martmi 3. t. $119.8 .1097 .7 \mathrm{St}$

6 H. Davidis. Die Darsds. Harfe. eor. Martini 3. I IIg f. 1092 . 5 Sr.

7 H. Cancellata. Die fchmalripfige Harfe. eod. 8. Chemn, 10. t. 152. \$. 1453. 7 St.

\section{Lade 79. \\ Cadus. Das Weinfars.}

1880

1881

1882

1883

3884

1885

1886

1887

1888

I C. Perdix. Das Rebbuhn. Gmel. Bue. cinum perdix. Ip. 3. Mattint 3. 6. 117. f. 1079 . 5 St.

2 C. Meleagris, Die gebandete Tonne mit Puncten. Gomel. Buccinum perdix. Sp. 3. I St

3 C. Coturuix. Die Wachrel. Gnel. Buceinum perdix. rp. 3. 6 St.

4 C. Cepa Dic gewăterte Tonne. Gmel. Buccinum olearium. ip. I. Martini 3. t. 117. f. 1076 - 1080.8 St.

5 C. Caffis. Das Stiickfals. Gmel, Buccinum dolium. Sp. 5. 3 St.

6 C. Galea. Die Biertonne. eod. 4 St. 7 C. Globofus. Die Kugeltonne. eod. I St.

8 C. Dolium, Dic bunte Tonne, cod. Martini 3. C. J16. f. 1073. 1074 . IOSt. 


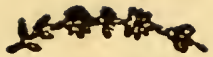

188919 C. Doliolum. Das buote Tönocbell. cod. $4 \mathrm{St}$.

$1890 / 10$ C. Albus. Die weille Tonne. eod. I St.

189 II C. Diapbanus. Die durchficheige Toa. ne mic zwey Mündungs-Säumea, eod. I.

\section{Lade 80.}

189212 C. Fascrarus. Die braun gebandere Tonue. Martini 3. t. 117 . f. 1081. 4 St.

189413 C. Pommm Die eyförmige Tonne. Gmel. Buccinum pomum. Ip 4 . Mar. tini 2. 36. f 370. 37 1. Kuore Verg. 6. 1. 23. 6. 2. $4 \mathrm{Si}$.

Cymbium. Der Kaho, oder die

$$
\text { Tepel-Bake. }
$$

I. Spire coroness. Die geksobse

$$
\text { Tepel-Bake. }
$$

1895 C. Acrbiopicrm. Der Mobren. Kahn. Gmel. Voluta zthiopica. fp.1 13. $8 \mathrm{St}$.

f. 784. St.

2 C. Perficum. Der petfifche Kaba. Marrini 3.t. 75. f. 785. 3 St.

3 C. Flanmeum. Die bunte Tepel-Bake. Maruni 3. t. 74. f. 780.4 St.

3 * C. Senticofum. Die dornigre Tepel. . Bake. Martini 3. t. 76. $.788 .2 \mathrm{Se}$. 


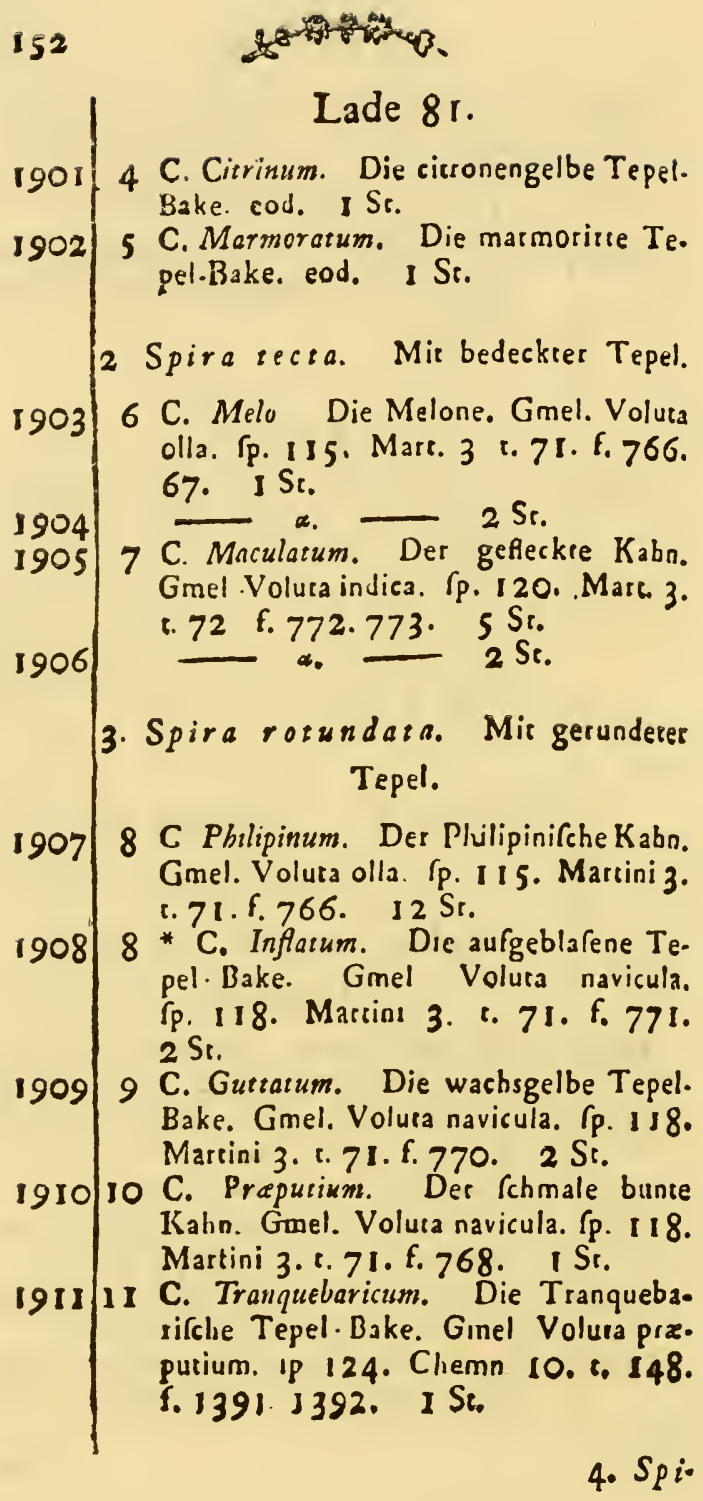


$1912 \mid \begin{gathered}\text { 4. Spira carinasa. Mir ausgekiehlter } \\ \text { Tepel. } \\ 12 \text { C. Facobinum. Die weifsausgekiehlte } \\ \text { Tepel Baks. Gmel. Voluta cymbium. } \\ \text { fp } 114 \text { Matciui 3.6. 70. F. } 762-65 . \\ \text { 3 St. } \\ 13 \text { C. Lagena vialoria. Die Reife Flafche. }\end{gathered}$ eod. 2 Sc.

191414 C. Cucumis. Des Kürbis. eod, Mars cini $765.2 \mathrm{Sc}$.

Lade 82.

I9I5 Is C. Cocblear Nepiuni. Der Neprunus.

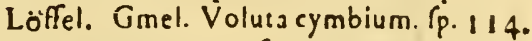

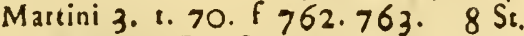

191616 C. Patera. Die Schale Gnel. Voluta Neptuni. Tp. 117. Mastini 3 1. 71. f. 767.3 St.

191717 C. Carinatum. Die ausgekiehlie Te. pel-Bake. Gmel. Voluca Neptuni. Sp. 117. Martini 3. t. 71 . 8. 767. 2 St.

5. Spira detrita. Mis abgellumpfret Tepel.

191818 C. Lineatum. Die liniirte Tepel-Bake. Gmel Voluta perficula. 'p 29. B. Martini $2 . t 42 \int 41.920$. 5 St.

191919 C. Lineara-punctatum. Die linèitse und punetirte Tepel Bake $3 \mathrm{Sr}$.

1920 20 C. Iuterreprum. Die Tepel.Bake mis abgefetzcen Linien. I St.

I92I2I C. Guttatum Die befprengte TepelBake. Gmel. Vuluta perficula. Ip. 29. a. Martini 2. $t_{0} 42$, f. 421 . 2 St. 
$3922 / 22$ C. Acbatmum. Die Agat Tepel-Bake. Gmel. Bulla cypre. Sp. 23. Knors 6. t. 4. f. 5. 4 St.

192323 C. Porpbyreum. Die Porphir Tepgl. Bake. tod. Martini 2. c. 65. f. 726. 727. 2 St.

23 a Marrioi 2. 2. 65. 6. 728. 729. 2 St.

192424 C. Cyliudraceum, Die Cilinder Tepel. Bake. ood. I St.

192525 C. Aurifiacum. Die orange Tepel Bake. Gnsel. Bulla cypsx. 'p. 23. 3 Si

\section{Lade I.}

\section{$B i v a l v e$ s.} $\mathrm{Z}$ weylchaaligee.

Solen. Die Meffer-Schaalen.

I S Siligua. Die Schore. Ginel. Solen filiqua ip. 2. Chemn, 6. t. 4. f. 29. Knorr Verg 6. 8. 7. f. 13 Si

2 S. Enfis Das Schwerde. Gmel. Solen enfis. fp. 3 Chemn. 6. t. 4 f. 30. Argenv. Cunch. \&. 24. f. L. 2 St

33 S. Gladius. Der Degen. Gmel. Solea vagina. ip. I. Chemn. 6. t. 4. f. 27. IO St.

44 S. Vagina. Die Scheide. Gmel. Solen vagina. ip. 1. Cherrn, 6. t. 4. f. 2628. Knars Verg. 1. T. 28. f. 3. 3 St. 


\section{Setaratos}

$5 \mid 5$ S Stlienla Das Scl:örchen. Gmel. So. len vagina. If. 1. Favaone $r$ LV.
fig. B. 2 Ise

66 S. Duticbos. Die gelle Seralil - Schore, dickfchaligt und viel abnliches von fol. gende No. $2 \mathrm{Se}$

77 8. Nicoburicus Die nicobarifshe Schote. Ginel. Solen maxinnis. Tp. 15. Cliemn. G. 8 5. f. 353 Se.

818 S Sivigularus Die rofenrothe Schoce. Gniel. Solen Atrigilatus. Sp. 7. Chemn. 6. 1.6. $\mathrm{f}_{41} 42.4 \mathrm{St}$

9 IS Lchticula Die linfunformige Schore. Gmel Solen ininimus: ip. 14. Cheinn: G. t. 5. f 31. a b. 2 St.

I0 10 S. Legunmen. Die Hülle. Gme! Solen legumen. Sp. 4. Chemn. 6.t. 5.f. 32-

II II S. Radiarus. Die geftrablee Scliote. Gmel. Solta radiarus. Ip. 6. Chemnn, 6. I. 5. f. $38-40.7$ St.

$1212 \mathrm{~S}$ Glycymeris. Die bäuchige K/2ffmufchel. Ginel. Mya giycymerts ip. 17. Chemo. 6.8 3.1.25. 1 Se.

\section{Lade 2.}

Laternula. Die Leuchte.

13 I I.. Truneata. Die abgeffumpfie Louchie. Gmel. Mya truicata. fp. 1. Chema. 6 . t. I. F. 1-4. Gualt. telt. s. 91. f D. 2 St.

I4 2 L. Anatina. Die Horn.Leuchce. Gmel. Solen anatinus. Ip 8. Chemn. 6.t.6. f. 46-48. Rumpf mur. s. 75. f. O. 3 S. 

Vulsclla. Der Kneiper.
I V. Major. Der grofíe Bart-Kneiper. Gmel. Mya vulfella. ip. 6. Chemn. 6. ז. 2. f. 1O. II. Knors Verg. 5. t. 2. f. $1-3$. I St.
2 V. Minor. Der kleine Bart - Kneiper. Gmel. Mya vulfella. Sp. 6. Chemn, 6. t. 2. E. 8. 9 . 5 St.

Lithophaga. Der Steinbohrer.

17 I L. Myzuloides. Der mufchelartige Steinbohrer. Gmel. Mytilus lithophagus. 5. 6. Chemn. 8. t. 82. f. $729 \cdot 7.30$. $4 \mathrm{St}$. braune. I St.

Mya. Die Miesmufchel.

1 M. Pifforum. Die Mablermufchel, davon 3 polirt. Gmel. Mya piđtorum. fp. ว. Clitmn. 6.t. 1. f. 6.8 St.

2 M. Margaritifern. Die Perlmufchel. Gmel. Mya margaritifera. ff 4. Chemo. 6. $t, f, 5,5 . S ?$.

2 a. dito politr, mit gefchnittenen Figuren. I St.

3 M. Perlata, Die Perlentrăgetin. I St.

Musculus. Die Mufchel.

I. M. Cygneus, Die grofle grüne Mufchel. Gmel. Mytilus cygaeus. Sp. I5, Chernn. 8.t. 85. . . $762.3 \mathrm{Si}$. 
$23 \mid 2$ M. Anatinus. Die grüne Miesmurchel. Gimel. Myrilus anatinus. 1p. 16. Chemn. 8. เ. 86. f. 763 . $2 \mathrm{St}$.

243 M. ComprefJus. Die Mahlet Strobm. mufcbel, alle defett. 3 St.

\section{Lade 3.}

254 M. Discors. Die queergeftreifre Mies. mufchel, die platte. Gmel. Mytilus dis. cors. fp. 21. Chemn. 8.t. 86.f. 767. I Se.

5 M..Nova Zeelandia. Die Neufeelandifehe Miesmufchel, die bauchigte davon drey. in der Haut, Gmel. Mytilus discors. fp. 2I. Chemn. 8. t. 86. f. 768. N2turfotth. IO. C. I. f. 8.4 St.

6 M. Modulaides. Die geftreifte Mies. mufchel, Gmel. Mysilus modiolus. fp. I4. Clietno. 8. t. 85. f. 760. is $\mathrm{St}$. a. $3 \mathrm{St}_{\text {。 }}$

7 M. Papuanus. Die papuanifehe Mies. mufchel. Gmel. Mytilus modiolus. fp. 14. Chemn. 8. t. 85. f. 758. I St.

8 M. Modulus. Die Modelmufchel. Gmel. Mysilus modiolus. \{p. I4. $\gamma$. Lifter t. 1057. f. 5. 3 St.

\section{Mytilus. Die Spizmulchel.}

1. Glaberrima. Glaste.

I M Ungulazus. Die ilaue Spitzmufchel. Gmel. Mycilus ungularus. $\int_{p} 12$. Cbemn. 8. t. 84. f. 756 . 5 St 
2 M. Variegatus. Die bunce $S_{\text {pitzmus. }}$ fchel Gmel. Mytilus verficolor. 'P. 30 . Chemn. 8. e. 84 f. 748 . 3 St.

3 M Violaceus. Die violenblaue Spitzmu.

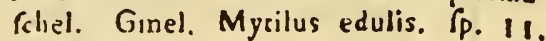
Chemn. 8. t. 84. f. $750.51 .3 \mathrm{Si}$.

4 M. Edulis Die Efsmufchel. Gmel. Mycilus edulis. Ip. II. I St.

5 M. Magcllanicus Die magellanifche Spirzmurchel Gmel Mytilus ungulatus. rp. 12 Cliemn. 8. t. 83 f 738. $\mathrm{ISt}$

6 M. PrClus Die buntlchāekigre Spitz. mufchel. Gmel. Mytilus afer. 'Tp 28. Chems. 8. 1. 83. f. 739. 3 St

f. 740 . Is.

7 M. Rofeus. ${ }^{\beta}$ Die sofenrothe Spitzmu. Schel. I St.

Mytilus. Die Spitzmufchel,

2. Sulsata. Gerille.

428 M.Sulcarus, Die getillse Spirzmurchel. Gmel. Mystlus bidens. Ip $13.4 \mathrm{St}$. - $\propto$ monfiós. Lilter t. 357. ז. 186. Knort Verg. 4. t. $30 \mathrm{f} \mathrm{3.} 3 \mathrm{~S}$ : Muschel Griel Mysilus bilocularis. 1p. 8. Chemu 8. 1 82. f. 736.4 St. $10 \mathrm{M}$. Purpureiss. Die Purpurmufcbel. I Se. 


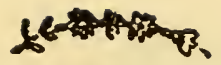

45 II M. Carinus. Die citronenfarbene Spizmufshel. Gmel. Mysilus cxuftus. 'p. 9. Chemn. 8. \&. 84. 8754 . ist.

12 Eine bis jetzt unbekanuce Perganent Miefsmufchel. ISt.

Pharetra. Der Kücher.

I P. Monoculoides. Die Pfoilnurchel. Chemn 10. 11 172. \&. $1675-77$. Naturforfcher 22 Ates St. i. 3.f. A-E. p. 23. I St.

\section{Lade 4.}

\section{Pinna. Der Schinken.}

I P. Gigas. Der grofle Schinken. Gmel. Pumna rotundata. Po. 5. Chemo. 8. 1. $93\{787$ i ist.

2 P. Bicolor. Der zweyłārbige Schinken. Gincl. Pinna bicolor. 'P. 13. Cheme. 8. 590 f. 780 . I St.

2 * P. Strizta. Det geftreifte Schinken. 1 St.

so

3 P. Cubernaculum. Der gerāucherte

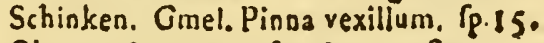
Chernn. 8. . 91. 6. 783. ISt.

$514 P$ Inflata Der angefchwollae Schiokein I St.

5 P. Virrea. Der durchlichtige Schinken. Gmel. Punna pectiuara. Tp. 2. variet. $\beta$. Chemn. 8. 6. 87. 6. 771 3 St.

$536 \mathrm{P}$ Rudis. Des fehwatze Sclinken. Gmel. Pinna sudis. IP. 1. 3. Chem. 8. . 88. f. 774 . I St. 
54 7 P Ferruginca. Der rothe schuppige Schinken. Gmcl. Pinna rudis. $\Gamma_{p}$. 1. $\boldsymbol{\beta}_{\text {. }}$ Chemn. 8. t. 88. f. 773. I St. 8 P. Violacea. Der blaue Schinken. I St.

9 P. Nobilis. Der kleine Cchuppige Schinkell. Gmel. Pinna nobilis. 'f. 3. Chenun. 8. 1. 89. f. $776.2 \mathrm{Se}$

Io P. Pectirsata. Der geflammte Schinken. Gmel. Pinna nobilis. \{p. 3 . Chemn. 8. r. 89. f. $775.2 \mathrm{St}$.

I I P. Pertiula. Der gefpickte Schinken. Gmel. Pinna nobilis Ip. 3. $\gamma$. Chem 8 . t. 92. f. 785. Knotr 2. t. 26. f. 2. 2 S:

I2 $P$ Elongara. Det Fchmale Schinken. Gmel. Pinna sudis. Sp. 1. Chemn. 8. t. 88. .773 . I St.

Beguina. Die Beguien-Nonne.

I B. Nepbritica. Die nierenförmlge Be. guin-Nonne. Gimel. Chana cordasa. fp. 8. 3. seniformis. Chemo. 7. t. 50. f. $502.3 .4 \mathrm{St}$

Mantellum. Das Mäntelchen.

I M. Excavarumi. Die Aufter des Fabricius. Gmel. Oftrea excavata. 'p'98. Chem.7. t. 68. f. 654. ISt.

2 M. Inflatum. Der Büuchigte halbe Mantel. Gmel. Oftrea inflata. Sp. 70. Chemn-7. r. 68. F. 649. 5 St.

3 M.Scabrun. Die Reibe Gmel Ontrea. glacialis. fp. 96. Chemn. 7. t.68. f. $652.53 .5 \mathrm{St}$. 


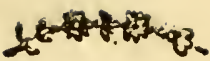

$64 \mid 4$ M. Lima. Die Feile. Gmel. Oftrea. lima. 'p.95. Chemn.7. t.68. f.65I. $6 \mathrm{St}$.

\section{Lade 6.}

Chlamys Der Mantel.

1 Glebre. Glatte.

- Serzata. Gefteifte.

I C. Cinnabarina. Dei zunobersothe Maptel. Gmel OAtea islandica. Ip. 55. Cbemn. 7. 5. 65. fig. 615, 616. 9 St.

2 C. Islandica. Der iständifhe Mancel. eod. 4 St.

- Sulcaza. Gefurchee.

3 C. Norwegica. Der norwegifche Mantel. Ginel. Oftrea citnoa. Ip. G2. Chemo. 7 t. 65. f. 618. 13 St.

3 * C. Solaris. Der gelbe Strablmantel. Gmel. Ottrea glabra ip. 50. von Born. t. 6. f. 4. 2 St.

4 C. Septentrionalis. Der nordifche Mar̈tel. Gmelio Oftrea glabra. 'pec. 50. $10 \mathrm{St}$.

5 C. Tranqueberica. Der ttanquebarifche Mantel. Gniel. OAtrea tranquebarica. 5. 67. Chemn. 7. 5. 67. 5. 647 . $4 \mathrm{Si}$.

IS. 

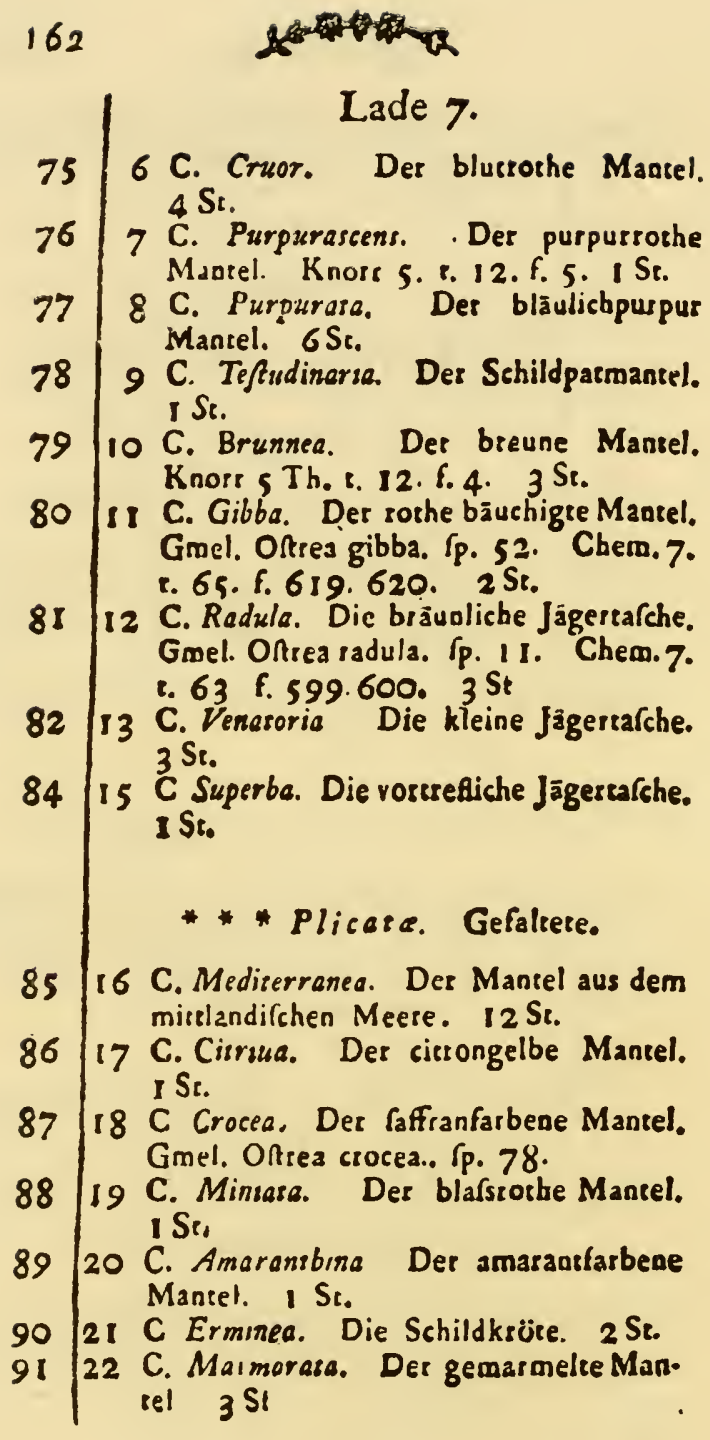
92123 C. Rubiginofa. Der rofffabene Mancel. Gmel. Oftrea glabra. Ip. 50. Chem 7. t 67. f. $643.2 \mathrm{Sr}$.

9324 C. Fuliginofa. Der braungelbe Manrel. eod. St

25 C. Punctulata. Der punctirte Mantel. eod 3 t.

26 C. Intertexta. Der gewirkte Mantel. od 1 St.

9627 C. Episcopalis. Der bifchöflich antel. eod. I St.

28 C. Tentorialis. Det bunctchàckigte Mantel. eod. 2 St.

29 C. Cafrenfis. Der Feldmantel. eod. 2 St.

30 C. Caprcina. Det Capuzınet Mancel. eod. 3 St.

\section{Lade 8.}

100

IOI

103

10233 C. Pallium. Der Herzogsmancel. Groel.

3I C. Cornea. Der hornfar Bige Mantel. Gmel. Ontea glabea ip. 50. Cham. 7. t. 67 f. 642 .

Chlamys. Der Manrel. 2 Scabriuscula. Raube. - Nodofa. Knotige.

32 C. Nodofa. Dic korall Dublecte. Gmel. Oresa vodofa. Ip 43. Chemn. 7. t. 64. f. 609. 6ia Knorr Veig. 1. t. 5. .. 1. \& 4.t. 13. f. 5.5 St.

* Squamara. Gelchupse. Oltrea pallium. Tp 40. Chemn. 7. ז. 64. f. 607. Knots Verg.2. t.2 I. f. 1. 2. IISt.

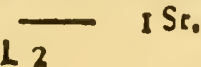


104134 C. Sulpluree. Der fchwefelgelbe Mantel. Gmel. Ontrea fulphurea. Sp. 64 . Chemn. 7 t 66.f. 629 . I Si

105 C. Cardinalis Der Cardinalsmantel. Gmel Oftrea potphyrea. Ip.65. Cbem.7 c. 67.6 .632 .4 St.

$80636 \mathrm{C}$. Autrantiaca. Der orange Mantel. Gmel OArea aurantia $\int_{p} 37.17 \mathrm{Sr}$.

10737 C. Gu/so. Der kleine Mantel. Gmel.

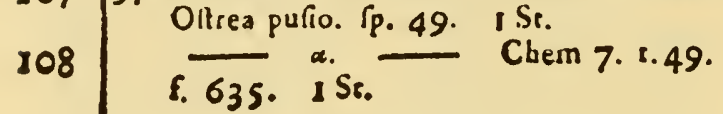

\section{Lade 9.}

10938 C. Varia. Der veranderliche Mancel. Gmel. Ontea varia. Ip. 48. Chem. 7. 2. 66. f. 633. $14 \mathrm{Sr}$.

110

39 C. Incerta. Det buntfchäckige Mantel. I St.

11 40 C. Nebulnfu. Der wolkigte Mantel. $3 \mathrm{St}$.

11247 C. Strinza. Der geftreifte Mantel. 1 St.

42 C. Lingua Felis. Die Katzenzunge. Schrörers Einleitung in die Cunch. 3 B. Seite 328. N. 15. Chema. 7. r. 66. f. 630. I St.

11443 C. Incarnata. Der blutrothe Mantel. Gmel Oftrea incarnata. Sp. 83. 2 St.

II 44 C. rfeudamufium. Det unăchte Compas. 2 St.

116

C. Papyracea: Der dünne Papiermantel. Gmel Ontea virrea. Sp. 66. ChemDitz f. 637 . to 2 St. 


\section{\begin{tabular}{c|c} 
Pecten. Die Rarnm-Mutchel. \\
I P.Maxima. Die gröfsie Kammmufchel. \\
Gmel. ORtea maxima. fp I. Chem.7.
\end{tabular} c. 60 f. 585 . Knorr. Verg. 1. t. 14. f. 12. \& 2.1. 14. f. 1.3. ISt. \\ 2 P. Fncobrea. Die Jacobsmufchel. Gmel. Orica jarobza. \{p. 2 Ctemn.7.t.60. f. $588.589 .2 \mathrm{St}$ \\ 3 P. Dubius. Die kleine Kammmufchel. cod. 3 St. \\ 4 P. Pigulus. Die bunte Kammmutchel. eod I St. \\ 1215 P. Zicanc, Der ziczac Kammmursbel. Grnel Oftea zicrac. 1p. 3. Chemn.7. t. 61. f. 590591.4 St. \\ t. 61 f. $592.2 \mathrm{Sr}$. \\ 6 P. Polisus. Die geglattere Kamm.Mu. fche!. 2 Se.}

\section{Lade IO.}

Amufann. Die Compas-Dublette. 1 A. Plenronectes. Die Compas Dublezce. Gmel Oftrea pleuronedtes (p. 6. Chem. 7 t. 6 r. f 595. Knotr Veig 5.t.20. f. 3. 4. I St.

2 A Faponicuma. Die lapanifche Compas. Dublette Ginel. Oftiea japonica. Ip 8. Chemn 7 \&. 62. f. 596.4 Sc. 3 A Magnericum Die gräulichrothliche Compas Dubleste. Gmel. Oftrea pleuronectes. Ip 63 St.

4 A I.oreuzzianum. Dic I.orenzifche Cumpas Dubletle Gmel. Onrea Lau rentii P. 7. Chemn. 7. 1. 61. ..593. 5942 St. 
5 A. Toftudinariun. Die Schildpatfatbene Compas - Dublette. I St.

\section{Lade I T.}

Ephippium. Der Sattel.

IE. Polonicum. Det polnifche Sattel. Gmel. Anomia fella. 1p. 27. Chemv. 8 . t. 79. f. 7 14. Knorr Verg. 4. 8. 18. f. 2 \& 5.t. 29 f. I. I St.

2 E. Placentn. Dei Kuchen. Gmel. Ano. mia placenia lp 26. I St.

3 E. Transparens. Der durchfichrige Sar. tel. Gmel. Arumia placenta. fp. 26. Chemn. 8. ז. 79, f. 7 i6. I St.

4 E. Anomia. Die braune TransparentMufcliel, oder der kleine papiene Englifche oder Fohlnitche Sartel. Chenn. 8. t. 79. f. 7 15. Knorr Verg. 2. 1. 24. f. I. Rumpl. Anbour. ז. 47. f. B. Onteum placentiforme. I St.

\section{Calopodium. DerLeiften.}

I C. Albidum. Derweife Leiften Emel. Tellina inxquivalvis, $f_{p} 23$. Cliemn.6. t. I1. f. 106. 2. b. c. d. I St.

Pinctada. Die Perlmurer.

I. Lata. Perlmutter-Schale.

I P. Margaritifera. Die Perlmuttet, eine davon ift polirt. Gmel. Mytilus margaritiferus. Ip. 4. Chemn. 8.5.80.f. 717721. a. b. Knorr Verg. 2. t. 25. f. I. 5 St. 


\section{Letop.}

$35 \mid 2$ P. Foliacea. Die blätrrige Perlmutter. Gnel. Mytilus margaritiferus. Sp. $4^{\circ}$ Knorr Verg. 4. 1 . I8. f. I. I St.

3 P. Coftantea. Die caftanienbraune Perl. mutter. Gmel. Mytilus margaritiferus. Ip. 4. I St.

4 P. Imbricara. Die fchuppige Perlmus. rer. Gmel. Myrilus margatitiferus. Ip.4. Chemi. 8. f. 7 19. Kagrs Verg. 2. 5,25 . f. 2. 3. 4 Se.

\section{Lade I 2.}

2. Prerigiesa. Vögelcheo.

5 P. Penguin. Der Penguin. Gmel. Mycilus hirundo. (p. 22 8) Chema, 8. r. 8I f. 728. Knors Verg. 6. 2. 2. f. I. I Se

1396 P. Colymbus. Die grofe Schwalbe. Gmel Mytiluz hirundo. Ip. 22. Chem. 8. 8. 81. . 723 . I St.

1407 P. Hirundo. Die Schuralbe. Gmel. Mytilus bitundo. Ip. $22 . \quad$ Chemn. 8. 8. 81. 6. $725.3 \mathrm{Sc}$

J4I 8 P. Brutrnea. Das braune Vögelchen. Gmel. Mycilus birundo. Ip. 22. I. Sr.

1429 P. Apus. Die Geierfchwalbe, defect. Gmel. Mytilus hiruado. ff. 22. I St

Pinctada. Die Perlmutter.

3. Elongasa. Hammer.

P. Malleus. Der Hammer. Gmel Ottrea malleus. Sp 99. Chemn. 8. 8.70. f. 655. Knors 3. 8. 4. f. I. Lifter . $219.6 .54 .3 \mathrm{St}_{0}$ 


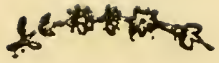

144 II I P. Regula. Das Lineal. Ginel. Ontea vulfella. fp. 100. Chemn. 8. 5. 70. f. $657.5 \mathrm{St}$.

Ifogorsm. Das Winkelmaars。

345 I I Norma. Das violet und weiste Li. neal. Gmel Oftreallognomun. (p. 125. y) Chemn. 7. t. 59.f. 582 . I Si.

2 I. Gromon, Der violetic Winkclbaken. Gmel Oftrea Ilogonum. Ip. 125. 8) Chemin. 7. t. 59. \&. 583 584. I $\mathrm{St}$

3 I Scapula Das Schultetblatt. Gmel. Oitrea ephlppium fp. 126. Chemn. 7. c. 58. f. 576 . Knorr 6. 1. 21 . f. I. 2 Sr.

4 I. Marfupinle. Die Tafche. Emel. OAtrea ephipplum. Ip 126. Chenin 7. t. 58 f. 577 . I Si.

149

5 I. Crenulatum Das gekerbte Schulterblatt. Gmol Oftrea ephippium. \{p. 126. I S.r.

340

6 I Alapbafiani. Der Faranengugel Gmel. Ofrea picta. \{p.127 Chemn.7.t.58. f. 575 . 2 St.

I5 I

7 I. Semiaurirum Der ohrförmige Flü. gel. Gmel. Oftrea perna. $f_{\mathrm{p}}$ 124. Chemn. 7.t. 59. £. 579.580. 3 St.

\section{Lade 13.}

Lopha. Der Hahnenkamm.

152 I L. Crifa Galli. Der Hahnenkamm. Gmel. Myrilus criftz galli. Ip. 1. Chem. 8. $6.75 \cdot f .683 .7$ St. 

$153 \mid \begin{gathered}684 . \\ 2\end{gathered}$ St. Chemn 8 t. 75.
$1542 \mathrm{~L}$ Hyotis. Der doppelee Hahnenkamm. Gmel Mytilus hyorss. Ip 2. Chernn \&. 75. f.685. Argens zoowiol pis 4.12. $f F 2 \mathrm{Sr}$.
3 L. Frons. Dar gefnitere Blate am häl- zern Sticl. Grunet Myrilus frons. Fp. 3. Cherma. 8. t. 75. f. 686. Knorr Verg. 4. t. 8 f. 3. I Si.
1564 L. Folium. Das Lorbecr-B'at:. Gmel. Ofrea folium $f_{f} 103$ Chentin. 8 . i. 7 I. f. $662-666$. Knors Verg. 10 t. 23 f. 2 I St.
1575 L. Curnu Cople. Das Füllhorn Gmel. Ofrea cornu copix. If 114 Chemn. 8. t 74. f 679. v. Born Sat Virdob. tett I G. F. 11. 12. 2 St.
158
159
6 L. Tbeca. Die Doie. Chema. 8. r. 73. f. 675.3 St.
60
161
7 L. Plicntella. Der dreyeskige grine Hahnenikarnm. 3 St.
Lade 14.
OArea. Die Aufer.

162 I O. Rbrnantoides. Die blaut gedrucke, Aufter. 2 St.

163

O Virgintana Der Niger Kathn Ginel. Oftrea viliginca. 1p. 113. Chemn 8 1. 73 f. 677.3 St.

16430 Prradamitica. Die Sündfuiths A4. fer. Ginel. Oftrea finenfis. Ip. 108. Chemn. 8.t. 72. f. 668. I St.

16540 Lincata. Die hiniiste Aufter, din douplete und zwey halbe. 4 St. 
$166 \mid 50$. Bilineara Die zweymal liniirte Aufter. Ontea edulis, Sp. 105. 8. Chem. 8.t. 7 I. t. 660.4 St.

6 O. Stellata. Die Stero-Aufter, 4 St.

7 O. Pedum. Der Schāferftab. äufferft sar. Favande Pl. 80. fig. K. I St.

\section{Lade 15.}

8 O. Edulis. Die efsbare Aufter. ORrea edulis. 'P. 105. E) Chemn. 8. t. $7 \mathrm{t}$. f. 66 r. Cbemn. 8. c. 74. 8. 682. $23 \mathrm{Se}$.

9 O. Elongata. Die lānglichte Aufter. eod. 3) Chemn. 9. 1. I i6. f. 99596. 3 St.

10 O. Silicula. Die kleine langlichre AaAter. cod.

\section{Lade 16.}

Mactra. Die Korb-Murchel.

I M. Glauca. Die geftrablie Korbmu. fchel. Gmel. Mactra glauca. 'p. 20. Chemn. 6.t.23. f. $232.233 .4{ }^{4}$.

$2 \mathrm{M}$. Meculoia. Die gefleckte Kbibmufchel. Gmel. Mattra maculara. fp. 16. Chemo. 6. t. 21. f. 208.209. ISt.

3 M. Corallina. Die blarstöthliche Korb. muifchel. Gmel. Madra corallina. Sp. 9. Chemn. 6.t. 22. f. 2 18.219. 2 St.

4 M. Lekfea. Die milchweiffe Korbmu. fchel. Groel. Mactra lactea. Sp. 10. Chemn. 6. t. 22. E 220. 221.3 St. Gmel. MaAtra violacea. Tp. 18. Chema. 6. t. 22. f. 213.214 . Koots Verg. 6. t $5 \cdot$ f. 2.6 St.

$17 \%$ 


\section{$24+2+20$}

1776 M, Stulsoruss. Der Narrenkarh. Gmel. Mactra ftultorum. Ip. II. Chemn. G. 2. 23. f. 224-226. Knors Verg. G. t. 5. f. I. 2 Si.

7 M. Striazula. Der geftreifre Korb. Gmel. Mactra ftriatula $\int_{p}$ 4. Chermn. 6. t. 2I. E. 205. 206. Knort Verg.6. t. 34. f. I. 2 Se.

7 - M. Bicordara. Der herzförmige Korb. $2 \mathrm{St}$

$1808 \mathrm{M}$. Pellucida. Der durchficheige Korb, Gmel. Mactra plicataria. Ip. 2. Chernn. 6. t. 20 f. $202-204.5$ St.

9 M. Spengleri. Der Speoglerfche Korb. Gmel. Mactra Speogleri. Sp, 1. Chemo. 6. t. 20. f. 199-20r. I St.

10 M, Rugofa. Der runzliche Korb. Gmel. Mactra rugola. Ip. 23. Chemn, 6. 1 24. f. 236.2 St.

183

II M. Lutraria. Det Fifchkorb, Gmel. Mactra lutraria. Sp. 14, Cbemn. 6. t.24. f. 240.24I. I St,

\section{Lade 17.}

Tridachnes. Die Nagel.Mufchel.

I T. Maxima. Die gröfte Nagelmafebel. Gmel. Chama gigas. Ip. 2. Chema. 7. t. 49. f. 495.3 St.

a. - Cheuro. 7. 49. f. $496.2 \mathrm{Sr}$.

2 T. Noce. Die Vater Noahmufchel. Gmel. Chama gigas. Sp. 2. Chemn. 7. ז. 49. f. 494 . 4 St.

3 T. Gigas. Die grofte Nagelmufchel. 189 Gmel. Chama gigas. Ip. 2. I St. 
$190 \mid 4$ T. Imbriensa. Die gedeckre N3ge?mu. ichel. Gmel. Chama gigas. fp 2. ist. 493. $6 \mathrm{Se}$,

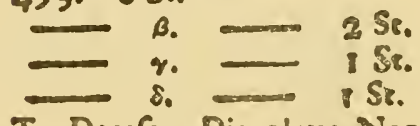

5 T. Derafa. Die glame Nagelmurbel. Gmel Chama gigas ip 2. f) Cbemn. 7 R. 49. ร. $49 \%$. 2 s.

\section{Lade 18.}

6 T. Hippopus Der Pferdehuf. Gmel. Chama hippopus. fp. 3. Chemn. 7. t. 50. f. 498.499 , St

$7 \mathrm{~T}$ Ungrile. Dee langlichre Pferdebuf, Gmel. Cbama bippopus. Fp. 3. Chem. 7. t 50 f 498.499. I St.

8 T. Arcinelle Der Itachliche Pferde. huf. Gme!. Chama arcinella. Ip. 14. Martinif. 522.523 . Knosz Verg. 6. c. 36. .. 1.2. 3 St.

Tuceta. Die Paftere.

I. T. Piloja. Die groffe Dickmufchel. Guel Area pilofa ip 36. Chemn 7. t. 57. f. 565 566. Knoss Verg. 2. B. 23. f.6. 8. 6. e. 12 f. 4.1 St.

200

$2 T$. Glicymeris. Die robhliche Panere. Gmel. Arcaglieymeris Tp. 35. Chem.7. i. 57. 5564 Knorr Verg. 1 t.21. f. 4.86 t. 14. f. 3.2 S. $3:$ Lenteginofa Die bunte Paftece. Gnel. Arca undata. Ip. 32 . Knorz Vs:3.6. :. 14 6. 4. 2 Si. 


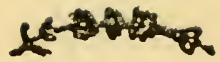

202 | 4 T. Undora Diegerellre Patese. Gmel. Aica unúzca. Tp.32. Ctren. 7. 4. 57. E 560.2 Se.

203

$5 \mathrm{~T}$. Imflase. Die bāuchigie Paflese. Gmel. Arca pilora. '́p. 36. I Sr.

204

6 T. Sinuasc. Die gentrabice Pafreie. Gnel. Aica angulora. 'p. 41. Cheirs. $\%$ c. 57 f $567^{\circ}$ I St:

205

7 T Mytiloides. Die murchelförmige Paftese. Groel. Arca glycymeris. Po. 35 . isi.

8 T. Peduncelus. Die gerillze Pallece. Gmel. Area pectunculus. ip. 33. Chem. 7. t. 58 . . 568569.4 St

Arca. Die Arche.

1. Pectuneulozdea. Kanmföronige.

I A. Cucullate Die Möochskappe. Gmel. Arca cuculius ip 23. Cbems. 7. t. 53. f 526-528 ist.

2 A. Ansiguata. Das Paquetboot. Gmel. Area antiquata. Fp. 16. Cheon. 7. 8. 55. f. 548 . Koorr Verg. 1. 1. 24 . 6. 3.4 .35 .

\section{Lade 20.}

209

210

3 A. Srapha. Die kahnformigè Arebe. Gmel Area anciquaca. Ip. 16. 2 St.

4 A. Nosabilis Die gezierce Arche. Gmel. Arca sntiguar2. 5. 16. Cbem.7. t. 55.8 .549 . $3 \mathrm{Se}_{0}$

211

5 A. Iraxgiusetuis Die ungleichfchalicbttauchigte. Arete Grol. Arca indica. ip $2 \%$ Chess. 7. t. 56. t. 552. $2 \mathrm{St}$. Si. Dito. $25 t$

Dic geftreckete 


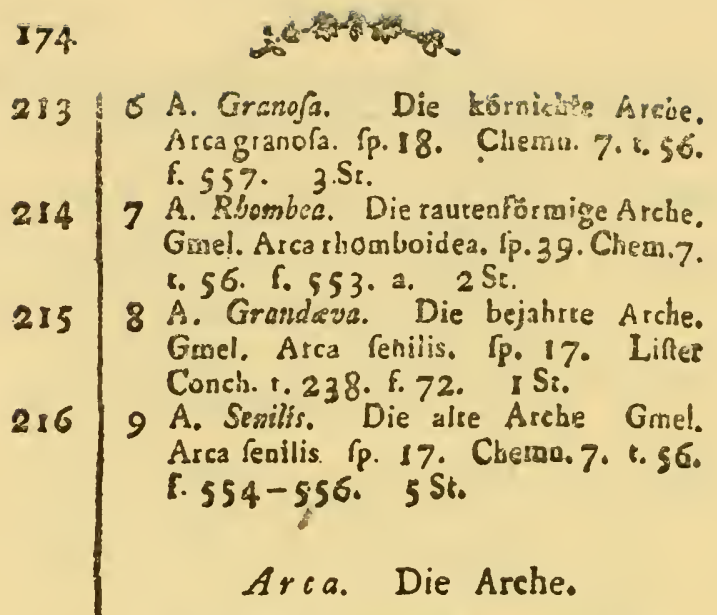

2. Vera. Die wabrea Arcben.

21710 A. Noes. Die Arche Noah. Gmel. Aica Nox. '́p. 2. Chemn 7. to 53. f. $529-31.25$.

II A. Patriarcbalis. Die uralie Arche. Guel. Atca.Noz. F. 2. Chemo. 7. 2. $53.8532 .13 \mathrm{St}$.

221

12 A. Martini Die Arcbe des Martini. Gmel. Arca toftrata. Ip. 8. Chemn. 7. t. 55 f. $550.55 \mathrm{r} .3$ St.

222112 A. Undase. Die wellenförmige Arcbe. Gmel. Arca roftrata. SP. 8. vas. ISt.

\section{Arca. Die Arche.}

3. Myaidea, Murchelförmige.

2.31 3 A Niuca. Die Tchneeweiffe Arche. Gmel. Arca ovara. '́p.6. Chemn. 7. : 54. 5338 . ISt. 


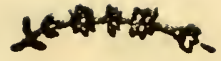

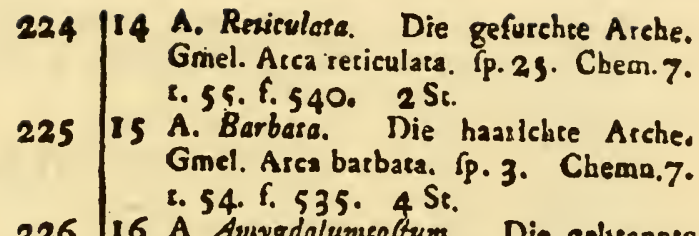

16 A Amygdnlumeoftum. Die gebrannte Mantel. Gmel. Arca barbata. (p. 3. r) Chemn. 7. t. 54. f.,534. 2 Ss.

17 A Magellanica. Die magellanilsbe Arche. Gmel Arca magellarica. CP. 24. Chema. 7. 1. 53. f. 539. I Sc.

Trifidos. Die gedrehte Arche.

I T. Arce tortuofa. Die gedrehte Arche. Gmel. Arca torcuofs. Ip. r. Chemn.7. 5 53. f. 524. 525. Knoss Verg. 1. C. 23. 1. 3. ISG

\section{Lade 2 .}

Paphia. Die Flügelmulchel.

229

230

1 P. Ala exis Die riefenmãfsige Venusmufchel. Sehr felten, und ein Exem. plar von aufferurdentlicher Grö́ffe. Gmel. Venus gigantea. (p. 89. Cheinn. 10. t. I71. 1. 166 1. Favanne Coach. 8. 49. 8. 91.2 St.

2 P. Ala papilionis. Der Schmetrerlings. fiügel. Gmel. Veous rocundara, $\int_{P} 134$. Chemn. 7. t. 42. f. 44I. Knors

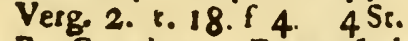

231

3 P. Gurtulare. Die gefleckse Flügel-

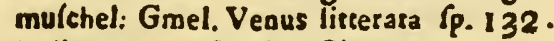
8. litterata confpetfa. Chemn. 7. 6. 42. f. 438 . I St. 
176

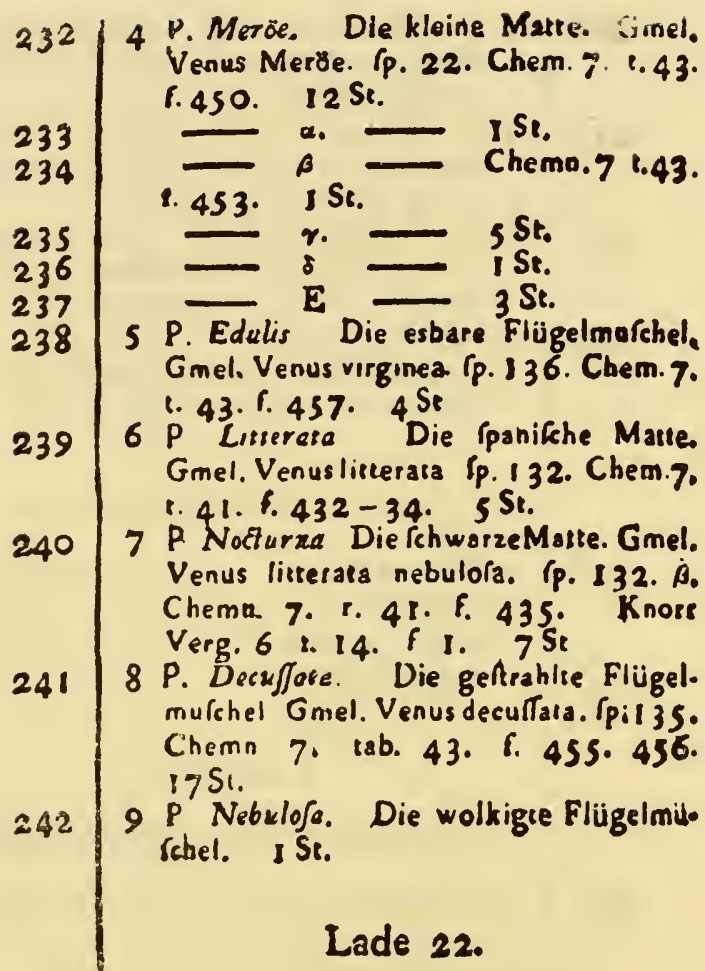

Gafrarium. Das Wafeleifen.

I G Fimbriarzm. Das weinte Wafeleifen. Gmel Venus fimbriata $S_{p}$. 15. von. Born. 1. 5. F. 4 . Chemn. 7. P. 3. vign 8. 20 t. 43 . f. 448.449 Lifter t. 355.1 .172 \& $\mathrm{L} 1056$. .f. I. $6 \mathrm{si}$

2 G. Tumidum. Das băllebigte Wa?telei. ren. Gouel. Venus pectinata. io. 78. Cberin. 7. 1. 39. 6. $415.33 \%$ 


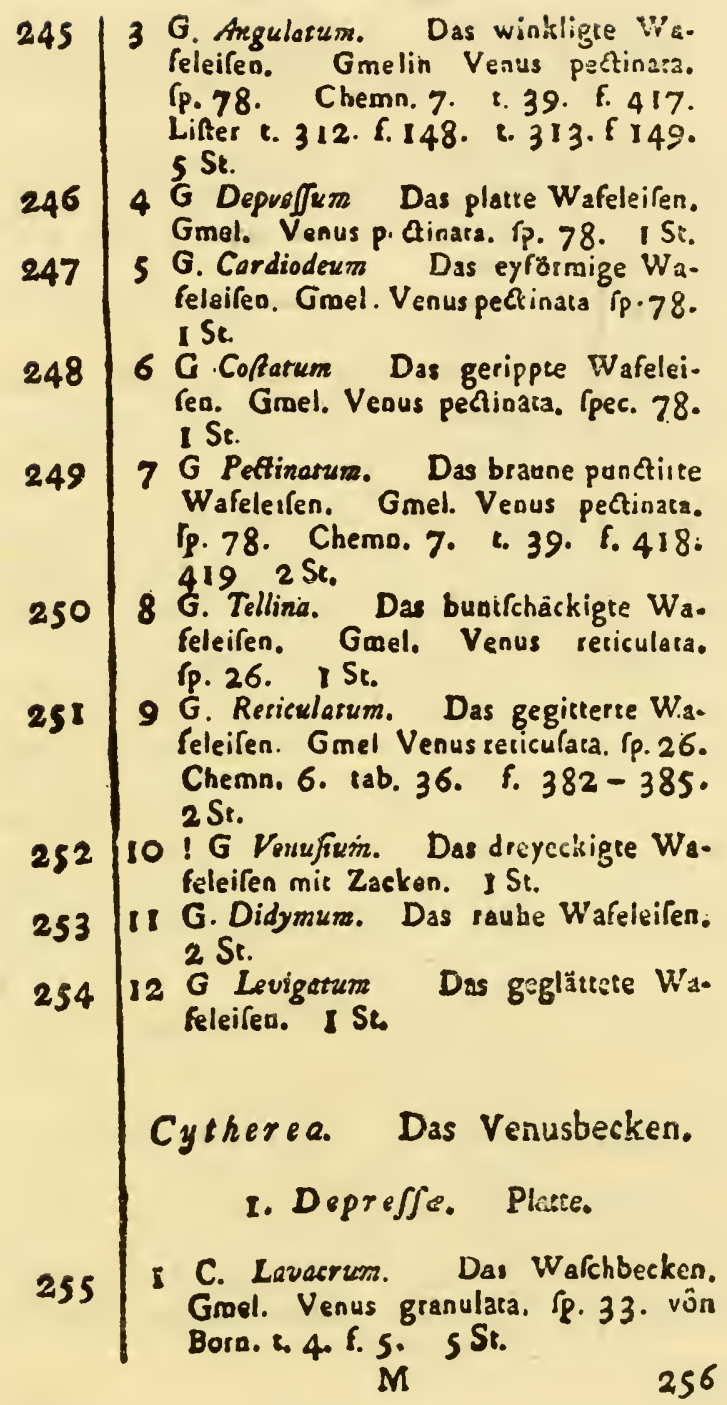




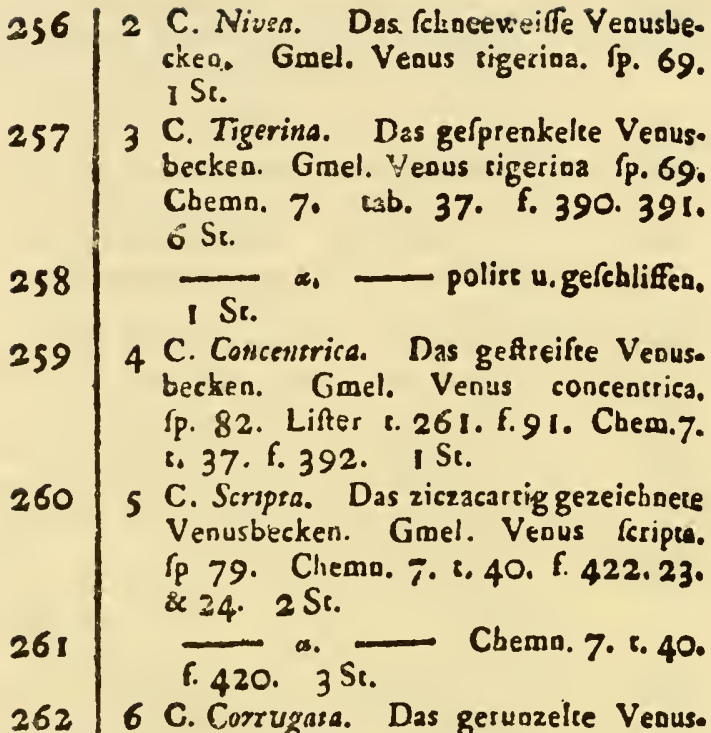
becken. Gmel. Venus foripta. 'p. 79: B.) Chemn. 7. 1. 39. f. 410.411. von Born. 8. 5. f \%. 5 St.

2637 C. Exfoleta. Das vesaltete Venusbecken. Gimel. Venus exfoleta. Ip. 75. Chemn. 7. e. 38. F. 404. Guals, te?. t. 7 5. f. D. 2 St.

2648 C. Obfoleta. Das veraltere buare Becken. Gizel. Venus Hiftrio. IP. 85 . Chemn 7. t. 38. 6. 407.2 St.

26; 9 C. Albida. Das weite Venusbecker. Ginel. Venus ezoleta. fp. 75. I St.

266

$10 \mathrm{C}$. Excifa. Das ausgerchnittene Venusbecken. Gmel. Venus finuata. Sp. 76. Chema. 7. t. 38. f. $400.401 .{ }_{1}$ St.

267 II C. Disriza. Das abgeoutzte Venusbe. stsen. St. 


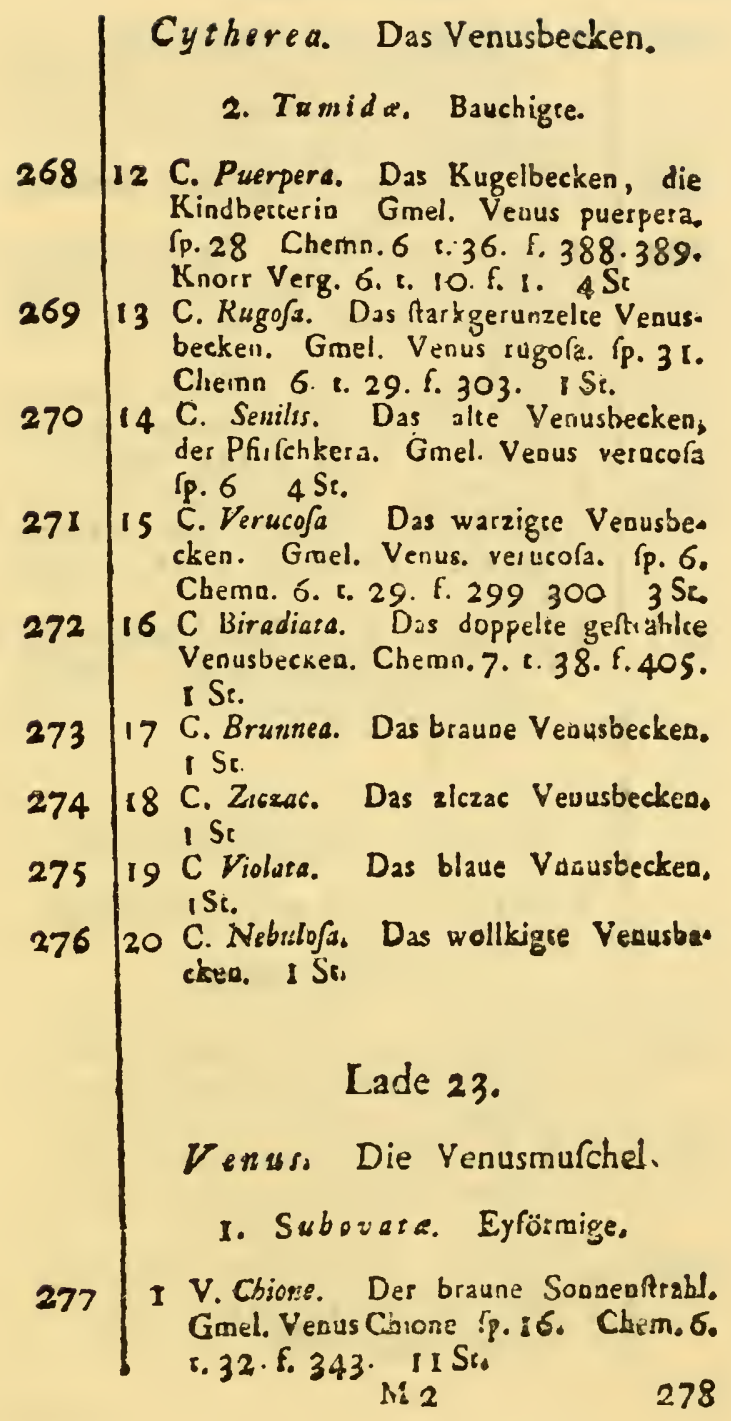


278 2 V Luforic. Die Spieldubletre. Gmel. Vequs Chione. (p. I6. B.) Chems. 6. t. 32. f. 3.40. inwendig bemahle uod vergoldet. $2 \mathrm{St}$.

279

3 V. Fuftigasa. Die geftäupte Veuusmuschel. 3 St.

280

3 *. Triradiasa. Die dreyntraklige Veciusmufehel. Gmel. Veaus eriradiata. fp. 45. Chemn. 6. tab. 34. f. 358 . 3 St.

281

282

4 V. Meculare. Die gefleckre Venusmuschel. Gmel. Venus maculaca. Ip. 17. Chemo. 5. t: 33 . f. $3456 \mathrm{St}$.

5 V. Ericyna. Dje unvergleichliche Ve. nusmufchel. Gmel. Venus Erycina. Sp. 13. Chemn. 6. T. 32 . f. 337. 5 St.

6 V. Sprume. Die unzehte Venusmufchel. Gmel. Venus Erycina. 'p. 13. Cbem, 6. 32. E. 338. ISto

\section{Denus. Die Venusmufchel.}

2. Triangularos. Dreyeckige.

$$
\text { * Glabra. Glatre. }
$$

7 V. Islandica. Die islandifehe Venusmufchel. Gmel. Venus Islandica. 'P. I 5 . Chemn. 6. 1. 32. f. 341 . $7 \mathrm{St}$.

8. V. Ferröenfis. Die fercionfifebe Veaus. mufchel. Gmel, Venus Islandica. 'p. I5. 2 St.

9 V. Pitar. Das Eisduplett, Gmel. Venus Islandica. (pec. 15. $\gamma_{0}$ ) Chemn. 6. 4. 32. E. 342. 2St。 

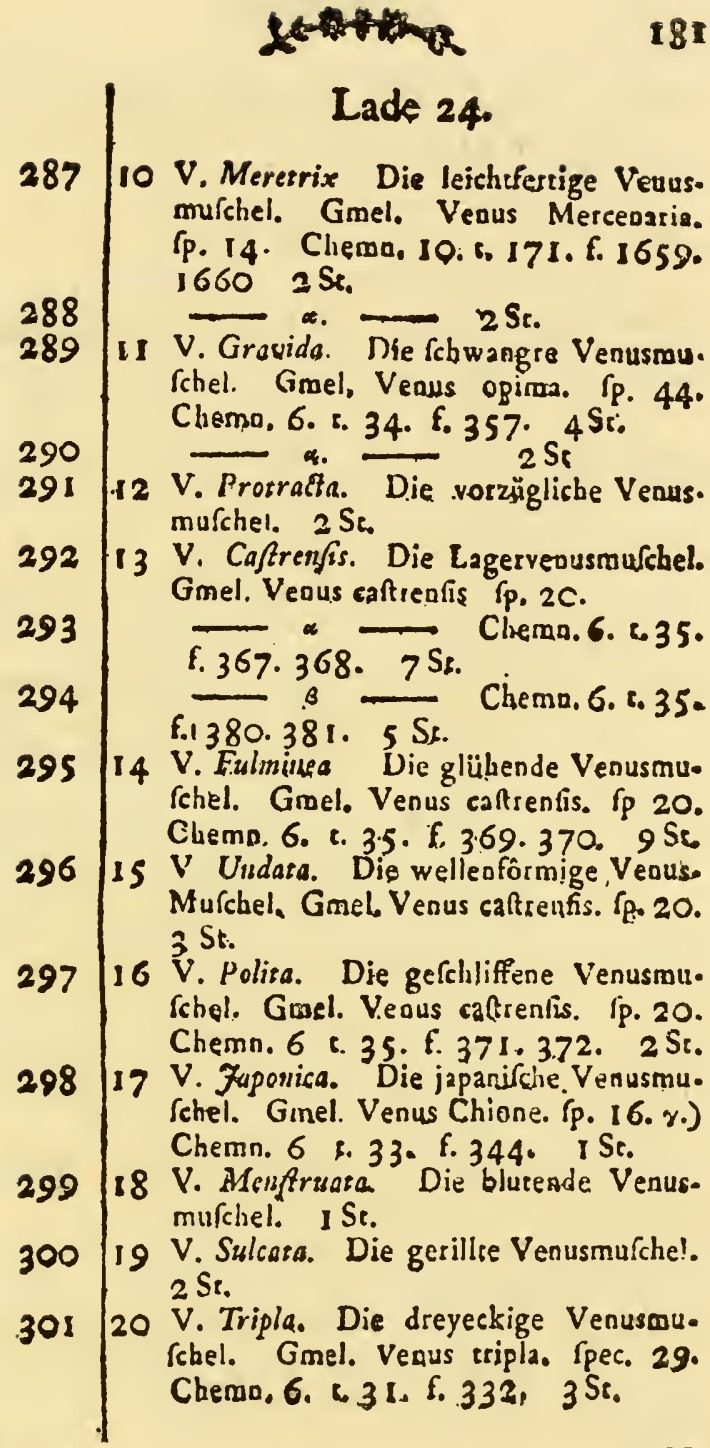

\section{Lade 24.}

Die leichertige Veuusmufchel. Gmel. Veaus Mercedaria. Sp. 14. Chema, 10: t. 171. f. 1659. 16602 St

II V. Grovida. Die febwangre Venusmu. rchel. Grmel, Veaus opirms. \{P. 44. Chemo, 6. 5. 34. f. 357. 4 St.

V. Protrafta. Die vorzügliche Venus. muschel. $2 \mathrm{Se}$.

V. Caftrensis. Die Lagervenusmufchel. $\longrightarrow$ Chema.6.6.35. f. 367.368 .7 St. f.1380.381. 5 S. Chema.6. 0.35 . V. Fulminea Die glühende VenusmuChel. Grael. Venus caltrenis. ip 20. Chems. 6. t. 3.5 . f. 3.69.370. 9 Se Mudara. Dig welleoformige, Veause Murchel, Gmel, Venus caftrenfis. [p. 20. 3 St. fchel. Grael. Veous cantrentis. Ip. 20. Chemn. 6 t. 35 . f. 371.372 . 2 Sr. V. Zaponica. Die japanif́che. Venusmu. Sehel. Ginel. Venus Chione. (p. 16. $y_{0}$ ) Chemn. 6 \&. 33- f. 344. ISt. murchel. I St.

V. Sulcata. Die gerillie Venusmurche!. 2 St.

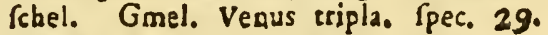
Chemo, 6. $31.5 .332,3 \mathrm{St}$. 
2. V. MnEtrois'es. Die Kcrbvenusmurehel. Gmel. Venus corbicula. fp. 39. Chem. 6. 2. 31 . E. 326.

\section{Lade 25.}

* Sulcata. Gefurchic.

22 V. Dione. Dieftachligre Venusmufchel. Gmel. Venus Dione. Ip. I. Chem. 6. r. 27. f. 27 I. 272 . Knorr. Verg. I. ข. 4. f. $3 \& 4$. 3 St.

23 V. Circinata. Die gereifte Venusmu. fchel. Ginel. Venus guineenfis. Tp. 10. Chemn. 6. ז. 30. f $31 \mathrm{r} .2$ Se.

24 V. Porcata. Die geftrahire Venusmu. feliel. Gimel. Venus ftriata. (p. 49. Chemn. 6 t. 34 f 365.66 . St. fchel. Gmet. Venus ftriata. Sp. 49. Chemn. 6. tab. 34. f. 365 . 366. ist.

26 V. Gallina. Die Henne. Gmel. Venus gallina. Ip. 9. Lifter t. 281 . F 119. i. 282. f. 120 . Chemn.6. 30. f. $308-310$. Knorr Verg. s. 1 i4. f $2-5.25 t$

27 V.Paphic. Das alte gerunzelte Weib. Gimel. Venus paphia. ff. 2. Chem. 6. t. 27. f. $274-76$ Knorr Verg. 2. t. $28 . r 2$ \& 6. .. 5. f. $6.9 \mathrm{Si}$.

28 V. Ebriofa. Die Schwelgerin. Gmel. Venus Dyfera Sp. 4. 1 Se. œ. I St.

29 V. Dyfera. Die breitblärrige Venusmufchel. Gmel. Venus dyfera. f́p. 4 . Lifer 5.297 . f. 1 :4. Chemn, 6. t. 97. 2\%9.280. Knotr Verg. 4. t.2\% f. 3.6.t. $10 f_{0} 2_{1} 2$ St. 
$313130 \mathrm{~V}$. Lamellara. Dic blattrige Venusmus rehel. Gnel. Venus dylera fp. 4 Chemn. 6. 1. 27. f. 280 . ISe.

$31 \mathrm{~V}$. Stritta. Die weille platte Venusmulcbel. Gmel. Venus dylera. Ip. $^{4}$. Cheran. G. c. 28 f. 289 I Sr.

$32 \mathrm{~V}$ Pacifica. Die Filedens-Flagge. Ginel. Venus plicata. If. 30. Chemn 6 . c. 28. f. 295-297. Valentin Abb. t. $15 \cdot$. 21 . $15 \mathrm{t}$.

33 V. Marica. Die gefeckre Venusmufchel. Gmel. Venus marica. ip. 3. Chemn. 6. t. 27. f. 282-284. Argenv. $200 \mathrm{~m} . \mathrm{r}, 12$ f. C. 5 it.

$34 \mathrm{~V}$ Clathrat.d. Die gegirterte Venusmu. Schel Gmel. Venus dysera. Sp. 4. Chemn 6. в. 28. f. 290.3 St.

$35 \mathrm{~V}$. Fluminea. Die grüne Venusmu. rchel aus lülsem Wallet. Chemn. 6 . c. 30. โ. $322.4 \mathrm{St}$.

$36 \mathrm{~V}$. Dunacma Die eckige Venusmu. Tchel. Gmel. Venus donacina. is 142. Knorr Verg. 6. t. 28. f. 7 . 5 St.

37 V. Roffraza. Die gefchnabelte Venusmufchel, kein duplere. Chemn. 10. t. $172,8,1670,1671$. ISt.

Donax. Die dreyeckige Mufchel.

1 D. Scorzum. Die Barth.Venus oder Venus-Prinzefs. Gmel. Donax fortum. fp. 1. Chemn. 6. 1. 25. f. 243-248. Knorr Verg. 6. t. 7. . . 7.. 4 St.

2 D. Serra. Die gezackre dreyeckige Mufchel, Gmel. Donax rugors. If. 3. Chemn. 6. 2ab. 25. f. 35x. 352 . $2 \mathrm{Se}$. 
$323 \mid 3$ D. Cureata. Det keilförmige Dreyeck. Grol. Donax cuneara. f́. 7. Chemn. 6. 2. 26. f. $260.9 \mathrm{St}$.

4. D. Tromeculizs Das Kibizchen Gimel. Donax irunculus. Sp. 4. Chemn 6. c. 25 f. 253.254 . Kuors Verg. 1. t. 7 t. $7.11 \mathrm{St}$.

5 D. Derticulata. Das gezäbnelte Dieyeck. Gmel. Dunax dentuculata. Ip 6. Cliema, 6 t. 26. f. 256.257 .6 St.

6 D Violacea Das violenblaue Dreyeck. Gmel. Donax denticulata. $\{$ g. G. I St.

\section{Lade 26.}

Corbula. Das Körbchen.

I C. Polygona Das winklige Kärbchen. Gmel. Tellina multangula, (p.9. Chem. 6. เ. 9. . 77.3 Si.

2 C. Anomala. Das unāchte Kä́bchen. Gmel. Venus deflorata. Ip. 24. Chem. 6.t. 9 t. $79.80 .2 \mathrm{Sr}$.

3 C. Rofca. Das rorhe Kürbshen. Gmel. Venus deforata. Ip 24. Chemn. 6. t. 9 f. 82.3 St.

4 C. Radiata. Das bunte Korbchen, zyvey Par tind gefchliffen und polirt. Gmel. Venus deflorata. ip. 24. Chemn. 6. 5. 9. f. 81. 4 St.

3325 C. Violacea. Das bläuliche Körbchen. Gmel. Venus deflorata. \{p. 24.2 St. $6 \mathrm{C}$ Porcata. Das gefurchte Körbchen. I St.

$3347 \mathrm{C}$ Purpurascens. Das purpurfarbene Kö-bchen, I St. 


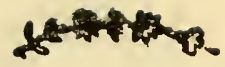

185

338 | 8 C. Rugofa. Das gerunzelte Körbchen. I St.

3399 C. Trmita. Das bāuchige Köıbchen. Gmel. Cliama oblonga. rp. 1O. Chema. 7. 2. 50.1.505. I St.

10 C. Angulara. Das winklige Kärbchen. I St

34 I

I1 C. Elongata. Das Isagliche Kôtbchen. I St.

12 C. Minuta. Das kleine Körbcher, Gmel. Solen minutus: fp. Ir. Chemn. 6. t. 6. f. 51.52 . I St.

13 C. Glass. Die Eichel-Dublette. Gmel. Chama calyculata. fp. 7. Chemn, 7 . t. $50 \mathrm{f}$ son.50?. I St.

14 Eine uchékannce Miuichel, dem aufern Anfchein eine Miesmurchel, dem Schlolle nach eine Arche, I Si.

\section{Lade 27.}

\section{Tellina. Die Telline.}

1. Lanceolasa. Lanzelförmige.

I T. Lifteri. Die Liferfebe Telline. Gmel. Telljna virgata. 'p. 3. Chem. 6. 5. 8. $f 73.2 \mathrm{St}^{\circ}$

2 T. Virgata Die gefrahlce Telline. Gmel. Tellisa virgara (p. 3. Chen. 6. c. 8. $66-72$. 6 St.

3 T. Virginca Die fchneeweife Teiline. Gmel. [ellina virgata. Sp. 3. 3 St.

4 T. Rofea. Die rolenrothe Tellipe. Gmel. Tellina roftrata. Ip 22. Chemn 6 . t. 10. f. 96 Koors Verg. 4. 8. 2. f. $3-5.2$ St. 
5 T. Spespieri. Die Spengleriche Telline. Gmel. Tellina Spingleri ip 30. Chens. 6. t. 10. f. $88-90$. 2 St.

6 T. Eoliacra Die Goldzunge. Gmel. Telline foliacea. Ip. 18. Chemn. 6. t. IO. 195.2 St:

7 T. Semulanaza. Rotbe Tulpẹblâtrer, Ginel. Tellina operculara: Sp. 22. Chem. 6.t. J1. f. 97. Knore 6.4 12. f. 1. 3 S.

8 T. Parcellamea. Die poreollan Tellioe. Gmel Tellisa radiata. Sp. 2 I. 9 St.

9 T. Rediana. Die droyftahlige Telline. Gmel. Tellina radiata. p. 21. Cisemn. 6. เ. II. . 102. IJnorr Verg. 1. t. 19. f. $1-4$. t. 2. f. 4. \& 4. t. 2. E. 2 . $35 \mathrm{St}$.

353 10 T. Ferröenjis Die Ferröenficebe Telline. Groel Tellina feryenfis. \{p. 3 i. Lifter Concis t. 394. 6. 24. 6. Chem.6. 8. 10. 8. 91.4 St.

354 it T.Ping Die gemahle Telline. Gmel. Tellina fervents $\int_{p} .3 \%$. I s.

355 12 T. Nebulufa. Die wolkige Telline. Crael. Tellina Servenfis. 1p. 3 I. $4 \mathrm{Se}$

13 T. Gerio Dię amethiffarbeac Telline. Grsel. Tellisa gari. 'P: 5, Chemn. 6. 8. 10. $f$ 93. Knors Verg. 6. \& 12. f. 2. 2 S.

14 T. Vialarea. Die blatse Telline. Gmel. Solen diplos. $S_{p} 13$ var. 3 St.

358 14 * T. Roprara. Die Kchrabelförmige Teline. Gmel. Solen diphos Ip. I3. Chemn. 6.t. 7 f. 53.54 .2 St.

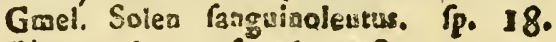
Cheñ. 6. $7,7,6 . \xi 6$. I St. 
$360 / 16$ T. Siriatzla. Die blastothe Telline. Gmel. Solen Atriarus. $\dot{1}$. 19. Cheinn 6. t. 7. $57.58 .2 \mathrm{St}$

36117 T. Corcinea. Die kleire rathe Telline. Gmel Tellina coccinea. Sp. 37. Chem. 6 ' 12 f. rog. 5 St.

36218 T. Coumplanaza Die placre Telline. Gmel. Tellina byalina ${ }^{p}$. 33 . Chemn. 6. t. 11 f. $992 \mathrm{Se}$

$36319 \mathrm{~T}$. Fragilis. Die dïnne Telline. Gmet. Telliaa fragilis $\}_{p} 6$. Chemo. 6. : $9^{\circ}$ f. 84.4 St.

$36420 \mathrm{~T}$ Deprc $\Omega$ a. Die plactgedrukte Telline. Gmel. Tellina depreffa 'p. 55 . Gualcieri teft. 8. 88. 6. H. J. 2. 4 Sto

\section{Lade 28.}

365

366

867

368

369

370

371

373

21 I. Angulofa. Die winklige Telline. Gmel Telliaa angulaca. fp. $_{4}$ Chem. 6. 2. 9 6. 74. 75. 4 St.

22 T. Ouars. Die eyförmige Telline. Gmel. Tellina indata. 'p. 8. Chemn.6. 6. 8 9. 86 . I Sh

23 T. Angulaza. Die eckige Telliae. 2 St.

24 T. Lavigara Die geglătrete Telline. Gmel. Tellina lævigata. [p. 20, Chem. 6. ז. 12. f. 111. Schtözts Eial. 2. p 649 t. 7. f. 10 $3 \mathrm{St}$.

$25 \mathrm{~T}$ Solaris. Die Sonentrahlen. Telline, 2 Sr.

$26 \mathrm{~T}$. Lingue felis. Die Raszerzunge. Gret. Telliua lingua felis. F. 2. Chem. 6.t. 8. 6. 65. Knort Verg. 2. t. 2. f. 1. $\mathrm{Se}$.

I. I St.

B. - rebrfelceo. ISt. 
2 C Humanum. Das Mencherlierz. Gmcl. Cardium cardiffa fp 2. Chen. 6 . t. 14. f. 145. I46. iSt.

3 C. Inflatum Das băuchigte Herz. Gmel. Cardium monftrofum. fip. 29. Favanne. PI. 5I. f. E. I. 2 St

3874 C. Dolorofumb. Das fcliwermuithige Herr. Gmel. Cardium monftrofum. Tp. 29. Chema. 6. t. 14. F. 149.150. 2 Si.

Fragum. Die Erdbere.

I F. Hemicardirum. Das dreyfeitige Hetz Gmelin Cardium hennicardium. fp. 5 . Cheran. 6. tab. 16. f. 159-16\% $3 \mathrm{Sr}$.

2 F. Flavum. Die gelhe Frdbere. Ginel. Cardium fragum. 1p. 13. Chemu. 6. r. 16. f. 166.167 .3 St.

3 F. Unedo. Die roethe Erdbere. Gínel. Cardium unedo. fp. 14 . Chem 6 . r. 16. f. !68. 169. Koorr Verg. 2. t. 29. f. 2.6 St.

4 F. Mediusmi. Die reife Erdbere. Gmel. Cardium medium. fp. 6. Chemn. 6.t. 16. f. 162-165. Knorr Verg. 1. 1. 29 . f. 5. 6. t. 20. f. 5. $4 \mathrm{St}_{\text {. }}$

\section{I.ade 30.}

Cardium. Die herzförmige Mufchel.

I. Lamellata. Blättrige.

I ! C.Cofatum. Das hochgerippre Herz. Gmel. Cardium coftatum. Ip. I. Lifter 1. t. 327. 164 . Knorr Verg.1. t. 28. f. 2. Cbema. 6. I, I5, f. Is I. 152. 25 . 
Cardium. Die herzförmige Murchel.

2. Coftnsis. Gerippte.

2 C. Lercoftomunt. Das gelbmündige Herz, oder Feirener Dublette. Gmel. Cardiurn magnum. Sp. 6. Linter Concin t. 33 1. f. 168. B.) Chem 6 . e. 17. f. 179. 2 St.

3 C. Aurantiacum. Das orange Heiz. 1 St.

$3954 \mathrm{C}$ Serrasum. Das gelbe Herz. Gmel. Cardiuin lavigatum 1p. 18 . Chem.6. 18 f. 189.3 St.

5 C. Fragile. Das dunfebalige Herz. $2 \mathrm{St}$.

6 C. Oblongum Das lãngliche Heız. Gmel. Cardium obiongun. ipec 34. Chernn. 6 t. 19. f. $1903 \mathrm{St}$

7 C. Arca. Das archenförmige Herz. St.

8 C. Grazlnndicum. Das Giönlandifche Herz. Ginel. Cardiuın grönlandicum. Sp.22. Chemn 6 t. 19. 6. 198. I St.

9 C. Kingens. Das Sperrmaul. Gmel. Cardium ringens. \{p. 3 I Chemn. 6 . t. 16. $\$ .170$ Knorr Verg. 4. t. 14. f. 3. \& 6 t. 3. f. 4. ISt.

10 C. Myoidrum Das Miesmufchelábraliche Heir. ISt.

402

II C. Lasum Die breite herzförmige Mufche!. Gmel Cardium larum. 'p. 36 . Chemn. 6 8. 19.f 192 193. I St.

12 C. Aeolicum. Das Oft - wad $W_{c}$ ftherz. Gmei. Cardium aolicum. Chemn, 6 . t. 18, f. 187,188 . I St. 
404 Ir 3 C. Tuberculssum. Die Carthagmenfifche Herzmufchel. Gmel. Cardiurn ruberculatum Pp. 11. Chem.6 t. 17. f. 173. Knort Verg. 5. t. 30. f. 2. \& 6. 1.8. f. 3. $2 \mathrm{St}$.

405

14 C. Cilialum. Die Islandifche Herzroufchel, 2 St. verlchiedene. Groel. Car. dium ciliatum. Sp. 10. Chemo. 6. 1. 19. 5. 195. 196.

Cardium. Die herzförmige Mufchel.

3. Imbricaice. Gedeckte.

406

I5 C. Ifocardia. Das gleichfeitige Zigel. Herz. Gmel. Cardium ifocardia. Sp. 12. Lifter ז. 323. f. 160 . Chemn. 6. x. I7. f. $174-176.2$ St.

407 I6 C. Campechienfe. Die gelbliclie Sägeribbe. Gmel. Cardium muricatum. Ip. I5. 4 St.

408

409

a. - Knort 4. t. 14 f. 5. Chemn. 6. t. 17. f. 178. I Sc.

17 C. Flavum. Das citronengelbe Herx. Gmel. Cardium flavun! 1p. 17. Schró. rets Einlete. in Conch. 2. t.7. f. II.

410 a. b. Bozann. recte. 2. f. 72. 2 St.

18 C. Celarum. Das gefurchte Herz. ISt.

\section{Lade $3 \mathrm{r}$.}

Cardium. Die herzförmige Mufchel.

4. Dentatce. Gezihate.

411

19 C. Muricarnm. Das Atachlige Herz. Gmel. Cardium aculearum. \{p. 7. Lifter c. 321. f. 158. Cbema. 6. t. 15. f. 155-157. Knort Verg. 6. t. 3. 6. 1. $8 \mathrm{Se}$.

$412-\alpha_{0}-$ ISt. 

41320 C Ecbinatum. Das donige lierz, die Egge. Gmel. Cardium ecipinacum. fp. 8 . Chemo. 6. t. 15. E. $156 . \& 157.158$. 159. $8 \mathrm{St}$.
Car a ins. Die herzförmige Murchel
5. Glabrs. Glatse.
21 C. Cor auritum. Die grofte Narrén- bapre. Guiel. Chama cor. Ip. I. Lifter Conch, t. 275. f. II I. Knorr Verg. 6. t. 8. E. I. Chemi. 7. t. 48 . . 483 . 4 St.
416 C. Corculum. Die kleirde Narrenkappe, : St.

\section{Lade 32.}

Anomia. Die Terebratel.

I A. Striatd. Dic geftreifre Terchratel. Gmel. Anomia doriata. Sp. 40. Chemn. 8. t. 78. E. 7 I0. $7 \mathrm{II}$. Naturforfeher 3. t. 3. f. $1-3 \cdot 3 \mathrm{St}$.

418

2 A. Bona Spei. Die Caprche Terebratel. Gmel. Artomia capenfis. fp. 35. Chem. 8. . . 77. f. 703. a. b. c. ISt.

3 A. Truncata. Die kleine abgeftumpfto Terebratel. Gmel: Anomia truncata. sp. I4. Cbemn. 8. 8. 77: f. 701. 2.b. $5 \mathrm{Sr}$.

4 A. Caput Serpentis. Der Schlangenhopf Ginel. Anonia Caput ferpentis. r.21. Chemn, 8. t.78.f.7I2. $8 \mathrm{St}_{6}$ 
421
422

423

$42 \dot{4}$

425

426

427

428

5 A. Vitria. Die durcbfichnge Tetebra. cel. Gmel. Anomia vittea $\int_{p} 38$. Chemn. 8. t. 78. f. 707. 708. Knors Vergn. 4. t. 30. f. 4. 2 St

6 A Coleoptratio. Die getügelse Tere bracel. 2 St.

Fen eft ella. Die Fenfterluke.

I F. Epbippiurs. Die fautedformige Fer. fterluke. Gnel. Anomia Ephippium. Sp. 3. Chemn. 8. x. 76. f. 692.693. Lifter t. 204. f 38. IOSt.

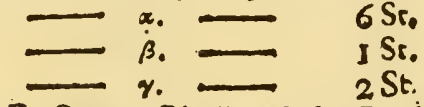

2 F. Cepa. Die Zwiebel. Gmel. Anomia Cepa. Sp. 4. Cbemn. 8. 5. 76. F.694. 695. Knors. Ver。6. t. 9. f. 5. $2 \mathrm{Si}$.

a. $\longrightarrow$ ISt.

3 F. Elecarica Die Berrftein Fenftesluke. Gmel. Anomia electrica. Is 5 . Chemn. 8 r.76. f.69 1. Knorr Vergu 5. 4. 25 . f. 6 . I St,

4 F. Squamula Die fchuppenförmige Fenfteilucke: Gmel Anomia fquamula. fp. 6. Chem. 8. 1. 77. f.697. isi.

Spondylus. Die Lazarusklappe.

S. Doninicenjas Die weifte Lazarus klappe mit hellrothen Wi bel. Gmel. Spondylus Grdaropus. Ip. I. 8. Chem.7. c. 45. f. 465 . Knors. Verga. 5. t. 9. f. I \& 6. เ. 9. f. I. 5 St. 
2 S. Rufus. Die braunrothe Lazarus. klappe. Gmel. Spondylus Gxdaropus. Sp. J. 2 Sr.

3 S. Regius. Die königliche Lazarusklappe. Gmel. Spondylus Gædaropus. fp. 1. Chemn. 7. tab. 46. f. 471 . 3 St.

435

4 S. Gadaropus. Die gezack:e Klappmurchel. Ginel. Spondylus Gxdaropus. fp. i. Chemn. 7. t. 44. f. 459 . Knore Verg. I. 7 f. I. 6 St.

5 S. Marisrubri. Die Lazarusklappe aus dem rothen Meere. Gnuel. Spondylus Gadasopus. [p. 1. 8. Chemn. 7. 2. 44 Martini f 460.4 Se.

438

6 S Labrum. Die Badewanne. Gmel. Spondylus Gzdaropus. Ip. I. I St.

$7 \mathrm{~S}$ Cruentazus Die blutrothe Lezarus. klappe. Gmel. Spondylus Gxdaropus. r. 1. $2 \mathrm{St}$.

\section{Lade 35.}

440

441

\& S. Macis. Die Muscsiblume. Gmel. Spondy us Gadaropus. Ip. I. I St.

9 S. Ducalis. Die brestblatrrige Lazerus. klappe. (imel. Spondylus Gxdarupus. (p. 1. o) Chenin.7. 1. 47. f. 476.477 . $3 \mathrm{Sr}$.

442

10 S. Variegatus. Die bunte Lazarus. klappe. (imel. Spondylus Gxdaropus. fp. 1. a) Chemn. 7 t. 45 . f. 464 . ist.

S. Pes-nfinzmus. Der Efelsfiufs. Gme!. Spondylus Gxuaropus. 's 1. छ) Cluem. 7. t. 46. f. 472.73 . I St. 
444 |r 2 S. Hyfrix. Der Igel. Gmel. Spondylus Gædaropus. (p !. p) Chemn. 7 . t. 45 f $470.2 \mathrm{Se}$.

13 S. Aurantiacus. Die orange Lazarus. klappe Timel. Spondyliss Gxdaropus. ip. 1. ६) Chemn, 7. t. 45 . f. 463 . $2 \mathrm{St}$.

$\propto$. Eingroftes Exemp. 14 S. Pifformm. Die hochrothe Lazarustslappe, eine rorisefiche Obcrichaale, allein. ISt.

15 S. Plicasus. Die gefaltete Lazarus. klappe. $3 \mathrm{St}$.

16 S. Criftatus Die kammförmige Lazaiusklappe, ISt.

Chama. Die Chama-Mufchel.

I C. Lazarus, Die Museatblüt e Gmel. Chama Lazarus IP 11. Knorr Verg. I. t. 8 f. 1 . Chemn. 7. t. 51. f. 507. 508. 2 St.

2 C. Crypboides. Die gefchnorkelte Felfenmuichel. Gnel. Chama gryphoides. 5. 12. Chemn. 7. t. 51. f. 510. 513. Knorr. Verg. 6. t I6. f. I. $6 \mathrm{St}$.

3 C. Maceropbylla. Die gelbe Chana. Gmel. Chama macerophyila. Ip. 17. Cbemn. 7. 1.52. f. 514-517. Knorr Verg. 1. t. 21. f. 2 \& 5. t. 24 . $t .1$. I St.

4 C. Lantellata. Die biătuige Chama. Gmel. Cliama bicornis. Ip. 13. Chem.7. 1. 52. I 519. I St.

5 C. Muricaia Die fischliche Chama. Gner Chama bicornis. If. 13 Cbem. 7. t. $52 . \digamma 518 \quad$ i St. 
Chiton. Die Käfermulchel.

T C Chitor Die Käfermurchel. I Se.

2 C. Brusueus, Die braune Kafeimu. Fchel Grnel. Chiton magellanicus. Sp. 12. Chemn 8 r. 95. f 797. 2 St.

4573 C. Squamofus. Die Rehuppenvolle Käfermulichel. Gmel. Chiron fquamofus. Sp. 5. Chemn. 8. 8. 94. f. $788-$ 791 Spengler Befchreib, berl Nacurf, I. F. 292 t 7. \& L.M. 7 St.

$4584 \mathrm{C}$ Vniriegatus. Die bunte Kà́etmutchel Gmel Chitenmarmoratus. Sy 15. Ghemin 8 t. 95. f. 805. 10 St.

4595 C. Cornetrs. Die hornfärbige, Käfermufonel. 2 St.

4606 C. Strmints Die gefreifte Kafermu. rehel I Sr.

461 7 C. Grifeo. Rribicundus. Die grautöthlichn. Kafermulchel. I St.

4628 C.. Viridis. Dıe grüne Käfermufchel. $2 \mathrm{St}$.

4639 C.. Magellanicus Die magellanifche Kafe! murcbel. Gmel. Chiton Magellani. cus. ip. 12. Chemo, 8. t. 95. 8.797. 798. 6 Se.

464 10 C. Granulofiss. Die braune granulitre Kafermufehel. Gmel. Chicon piceus. fp. 17. Chenin. 8. s. 96. f. $807^{-}$ 

810. Chem. Chit. t. 2. โ 626 . a b. c. 6 St. 11 C. Atro-Virsdis. Die dunkelgrüne
Káferroufcliel. I St.

\section{Lade 38 .}

\section{Lepas. Die Seetulpe.}

I L. Dindema. Die Wallfirch pocke. Giocl. Lepas Diadema lp 4. Lifter Conch. t. 445 . f. 288

Boen Mur. cre. Vind telf. t. I. f. 50 6. \& 9.7. f. a. Chema. 8. i. 99. f. 843.844 . I St.

2 L. Tinzinnabulum Dic grofie Seerulpe. Griel Lipas Tinzinaabulum $r_{p} .3$. Lifter t. 443. 1.285. Knorr Verg. 2. 1. 2. f. 6. \& 5. ?. 30. f. T. Chens. 8. i 97. f 828-83 I. 10 St.

4683 L. Rofata. Die rofenroche Seęrulpe. Gmel, Lepas Tintınnabulum $\beta$ Tulpia a!ba. Chemo. 8 t. 97. f. 832.

4 L. Sensicofa. Die mic Spizzen beferze Seerulpe. I St

5 L Spinofa. Die dornigte Seerulpe. Gmel. Lepas Spinofa. Sp. 22. Chemrs. Schr. berl. Nasurf. 5. i. 5. f. 3. 4. Chemo. Conch. 8. t. 98 . f. 840 . \& t. 99. f. $84 \mathrm{I} \cdot 2$ St.

471 6 L. Echimata. Die ftaclslichte Seetulpe. Gmel.Lepas f́pinofa. fp. 22. Chem.8. t. 98 . f. $840.6 \mathrm{Sc}$.

4727 L. Ovata. Die eyrunde Seetulpe. 3 St.

4738 L. Tefesudinaria. Die SchildkrörenSeerulpe. Gmel. Lepas teftudiartia. 4p. 6. Knorr Verg. 3. 4. 30. f. 3. 4. I St. 


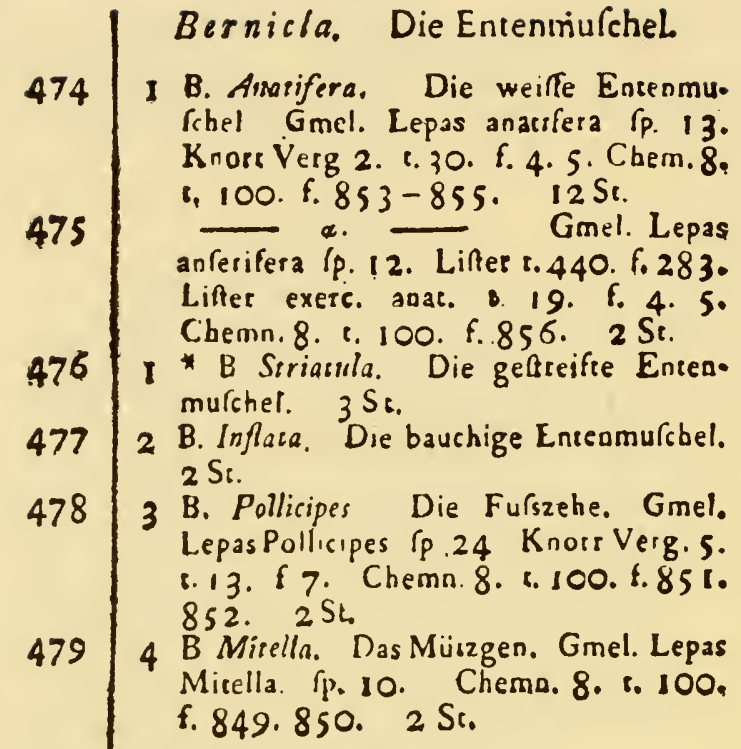

1 P. Coftatus. Die gerippte Pḥolade. Gniel. Phoias coftaca ip 2. Knorr. 2. t. 25 . f 4, Chemn.8. 1.101. 6.863. I $\mathrm{Si}$

$2 \mathrm{P}$ Dactylus. Die fchmale Pholade. Gmel. Puolas Dactylus ip I. Chem. 8. 8. 101. \& 863 I St.

3 P. Orientalis Die orientalifshe Phoo lade. Gmel. Pnolas orientalis Ip 7. Lifler 431 . fi 274 . Chemn. 8. 8. 101 . f. 860 . Walch Nacurf. 13. P 86. 1. 3. f. 15. I St, 
$483 \mid 4$ P. Crifparus. Die Lockenpholade. Gmel. Pholas crifputa. Ip. 6. Liftet t. 436. 1. 279. Chemn. 8. 1. 102. f. 872.873 . I Sc.

4845 P Pufillus. Die kleine Pholade, davon 2 Scücke fich ios Holz gebohreet. Gmel. Pholas pufilla. Ip. 5. Cherno. 8. c. 102. f. 867-871. 5 St. 
Oarded 
AIbula ...... p. 20

Ampulla ..... p. 110

Amusium ...... p. 165

Angarla ...... p. 71

Anomia ...... p. 192

Arca ........ p. 173

Architectonica. p. 78

Argonauta ..... p. 70

Astrasa ...... p. 79

Beguina ...... p. 160

Bermicola ..... p. 198

Buccimum ..... p. 117

Burla ........ p. 15

Bursa ........ p. 128

Busycon ....... p. 149

Cabestana ..... p. 130

Cadus ........ p. 150

Calopodium .... p. 166

Cantharus ..... p. 132

Cardium ....... p. 189

Cassis ........ p. 28

Cerion ........ p. 90
Chama ....... p. 195

Chiton ...... p. 196

Chlanvs ..... p. 161

Cidaris ...... p. 84

Cochlis ..... p. 146

Colus ....... p. 15

Corbuza ..... p. 184

CorcuIum .... p. 188

Cucullus .... p. 37

Cymatium ..... p. 129

Cymbium ...... p. 151

Cyphoma ...... p. 21

Cypraea ...... p. 22

Cytherea .... p. 177

Dentalium .... p. 68

Distorsio .... p. 133

Donax ........ p. 183

Drupa ....... p. 55

EIlobium .... p. 105

Ephippium .... p. 166

Epitonium .... p. p 91

Fenestella ... p. 193 

Ficus .......... p. 148

Fragum ........ p. 189

Fusus ......... p. 118

Gafrarium ..... p. 176

Galeodes ...... p. 53

Haliotis ...... p. 13

Harpa .......... p. 149

Helix ......... p. 106

Isogomum ...... p. 168

Janthina ...... p. 75

Lagena ........ p. 131

Lambis ........ p. 61

Lampadion ..... p. 77

Laternula ..... p. 155

Lepas ......... p. 197

Lithophaga .... p. 156

Lopha ......... p. 168

Iunatica ...... p. 101

Iunella ....... p. 103

Mactra ........ p. 170

Mantellum ..... p. 160

Nitra ........ p. 135

Morum ......... p. 53

Murex ......... p. Iflu
Musculus ...... p. 156

Mya ........... p. 156

Mytilus ...... p. 157

Nassa ........ p. 132

Nautilus ...... p. 70

Nerita ........ p. 16

Neptunea ...... p. 115

Nucella ....... p. 130

Oleacine ....... p. 110

Ostrea ........ p. 169

Paphia ........ p. 175

Patella ........ p. I

Pecten ........ p. 165

Pharetra ...... p. 159

Pholas ........ p. 198

Pila ......... p. 145

Pinctada ...... p. 166

Pinna ......... p. 159

Planorbis ..... p. 72

Plejona ....... p. 59

Plotia ........ p. 95

Pomaria ....... p. 73

Porphria ...... p. 32

Pterygia ...... p. 51 
, ... .

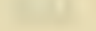

$+\cdots,+$

, , , , , , , .

, . . ...

, .., , , ,

$+\ldots+\cdots$

,,.,,.+

.....

- เ....,

- $\rightarrow,+, 0$

?

$, \ldots, \ldots$

$\cdots, \cdot+$

$+, \ldots, \cdot$

....

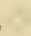

,

.., , .

, 3. 3 . 
Pupa ......... p. 110

Purpura ....... p. 139

Pyramis ....... p. 53

Pyrene ........ p. 134

Pythia ....... p. 105

Rapa ......... p.

Serpura ....... p. 69

Sinum ......... p. I

Solen ......... p. 154

Spondylus ..... p. 193

Strombus ...... p. 96

Syrinx ....... p. 121

Telline ...... p. 185

Terebellum .... p. 135

Thais ........ p. 54

Thiara ........ p. 109

Tibia ........ p. 123

Tridacnes ..... p. 171

Tritonium ..... p. 125

Trochus ...... p. 80

Tuceta ....... p. 172

Tudicla ....... p. 145

Turbo ........ p. 86
Turris ....... p. 123

Vasum ........ p. 56

Vemus ........ p. 179

Verpa ........ p. 69

Vexillum ...... p. 138

Volema ........ p. 57

Volva ........ p. 21

Vulsella ...... p. 156

Xancus ....... p. 134 














$$
1213^{0}
$$






, 
